\title{
Broad-band BVRI photometry of isolated spiral galaxies ${ }^{\star}$
}

\author{
H. M. Hernández-Toledo and S. Ortega-Esbrí
}

\author{
Instituto de Astronomía, Universidad Nacional Autónoma de México, Apartado Postal 70-264, 04510 México D. F., México \\ e-mail: [hector; sortega]@astroscu.unam.mx
}

Received 27 February 2008 / Accepted 14 May 2008

\begin{abstract}
Context. Uniform and high resolution observations in samples of isolated galaxies are required to estimate fundamental morphological and structural parameters for comparative studies of environmental effects and for confronting model predictions of galaxy evolution. A subsample of The Catalog of Isolated Galaxies (CIG), Karachentseva (1973), has been uniformly observed at San Pedro Mártir National Observatory in México and a photometric and morphological study of these galaxies has been carried out.

Aims. We report multicolor broad band (BVRI) photometry for a subsample of 40 isolated spirals drawn from the CIG. Total magnitudes and colors at various circular apertures as well as a detailed morphological analysis that is extended into the NIR bands are presented. Some structural parameters are estimated from the global light distribution in the optical and NIR bands to complement our morphological analysis and several correlations between the photometric and structural parameters are explored. Emphasis was given to the detection of morphological distortions at high/low intensity levels.

Methods. The observations, data reduction, and analysis are described. Morphology is reevaluated from a combination of optical logarithmically scaled $R$ band images, filter-enhanced $R$ band images, $(B-I)$ color index maps, archived near IR $J H K$ images from the TwoMicron Survey and optical-NIR $\epsilon$ and PA radial profiles after an isophotal analysis. $R G B$ images from the SDSS database were retrieved when available to complement our analysis. The $C A S$ structural parameters (Concentration, Asymmetry, and Clumpiness) were calculated from the images in each band.

Results. The fraction of galaxies with well-identified optical/near-IR bars (SB) is $40 \%$, while another $25 \%$ shows evidence of weak or suspected bars (SAB). The sample average value of the maximum bar ellipticity is $\epsilon_{\max } \approx 0.35 .57 .5 \%$ of the galaxies in the sample show rings. The CAS parameters change with the observed band, the morphological type and global color. We found 9 isolated galaxies with disturbed morphology. After reviewing their local number density and tidal strength parameters, we identified CIG 744 as a morphologically disturbed isolated galaxy having $M_{\mathrm{HI}} / L_{I}>1$; a tentative candidate to interact with a dark gas-rich object. However, its loci in the $\mu_{0}^{\mathrm{I}} / \log \left(M_{\mathrm{HI}} / L_{I}\right)$ diagram, suggests that CIG 744 is itself a gas-rich disturbed-isolated galaxy probably in a lengthy or ongoing process of formation, instead.
\end{abstract}

Key words. galaxies: spiral - galaxies: structure - galaxies: photometry - galaxies: interactions galaxies: fundamental parameters - galaxies: general

\section{Introduction}

The origin of the morphology of galaxies is one of the fundamental pieces to understand galaxy formation. One of the legacies of surveys like the Sloan Digital Sky Survey (SDSS) is that now we have a more extended view of the local universe allowing to look for fundamental relations between physical properties of galaxies at local and larger-scale environment. Recently, Park et al. (2008) used the SDSS data and studied the environmental dependence of the observed morphology at different smoothing scales to address the question of whether galaxy morphology depends primarily on the large-scale environment in which the galaxy initially formed, or on a smaller scale environment that may reflect the influence of later evolutionary effects such as galaxy-galaxy interactions. Park et al. (2008) pointed out that galaxies statistically become more isolated if they recently merged, and that at a fixed large-scale density more isolated galaxies are more likely to be recent merger products. It is therefore possible that the disturbed isolated ones are galaxies that recently experienced mergers.

An alternative explanation for finding disturbed morphology in isolated galaxies comes from the results of cosmological

\footnotetext{
* Appendices A and B are only available in electronic form at http://www . aanda.org
}

numerical simulations within the CDM model that show that a high population of subhalos survive inside galaxy-sized halos (Klypin et al. 1999; Moore et al. 1999). These sub-halos and the associated gas clouds could produce signs of morphological distortion on isolated galaxies (Trentham et al. 2001; Pisano et al. 2002)

These results emphasize the need of a careful identification of galaxies in isolated environments that may show evidence of disturbances not associated to intrinsic processes. Recently, Karachentsev et al. (2006, 2007), searched for disturbed (interacting) objects among very isolated galaxies. They inspected the galaxies in the Catalog of Isolated Galaxies (Karachentseva 1973, hereafter CIG) from the blue POSS-II images available on the digital Sky Survey, finding 5/1050 strongly disturbed galaxies and suggesting that the existence of "dark" galaxies could explain the observed signs of distortion.

An equally important problem, apart from identifying disturbed galaxies in isolated environments, is that of a detailed morphological classification of the isolated galaxies themselves. It is actually not clear whether or how far the morphological trends observed in clusters or groups extend to an environment typical of isolated galaxies.

The CIG is one of the most uniformly selected and complete samples of local isolated galaxies available in the literature. 
Since its publication, the CIG has shown its usefulness for studies either of individual or statistical nature. Although recognized as a good comparison sample for studies on environmental effects, it has not been until recently that a careful revision of their main properties, and in particular, of its morphological content has begun. Sulentic et al. (2006) inspected the galaxies in CIG from the red POSS-II images available on the digital Sky Survey providing a uniform classification for these galaxies.

The aim of this paper is to present a global BVRI photometric and morphological analysis for 40 galaxies from the CIG based on new uniform and deeper CCD observations. We applied a uniform reduction pipeline and an image processing scheme to produce an homogeneous set of photometric and detailed morphological data. We take advantage of the higher resolution $\left(\sim 0.5^{\prime \prime} /\right.$ pix $)$ and higher dynamic range of our images to proceed with a new morphological revaluation for these 40 isolated galaxies that is extended into the NIR bands, giving special emphasis to the detection of low/high surface brightness morphological distortions. Further we carry out a structural analysis in the optical and NIR bands through the concentration, asymmetry and clumpiness $(C A S)$ parameters, that complements our morphological study. A detailed morphological and structural characterization of galaxies in isolated environments is also useful for studying intrinsic secular processes that are able to affect the structure and dynamics of galaxies, for instance, the formation and evolution of bars, rings, lopsidedness, and bulges.

The outline of the paper is as follows: Sect. 2 describes the observations and reduction techniques used. We also present a comparison of our estimated total magnitudes against those in the literature. Section 3 presents a correction scheme for our apparent and absolute magnitudes and colors. In Sect. 4 we discuss the observed morphology based on logarithmically scaled $R$-band, $R$-band filtered-enhanced images, $(B-I)$ color index maps, composed near-infrared (NIR) $J H K$ images extracted from the Two Micron All Sky Survey (2MASS) archives, optical/NIR $\epsilon$ and PA radial profiles after an isophotal analysis and $R G B$ images from the Sloan Digital Sky Survey (SDSS). In Sect. 5 we present the results of the optical morphology with emphasis on the detection of distorted morphology in isolated galaxies. In Sect. 6 we present our estimates of the structural $C A S$ parameters in the optical $(B V R I)$ and NIR $(J H K)$ bands. The loci of our isolated galaxies in the $C A S$ diagrams space are presented. Here we also explore some basic correlations between the photometric and structural parameters in this sample that could be useful for comparative studies involving galaxies in other environments. Section 7 is a summary of the main conclusions achieved. An Appendix is devoted to the presentation of $B V R I$ magnitudes at two other concentric circular apertures.

\section{The data sample}

\subsection{Isolated spiral galaxies from the Karachentseva catalog}

We have carried out an observational program at the Observatorio Astronómico Nacional at San Pedro Mártir (OANSPM), Baja California, Mexico, devoted to obtaining uniform and deep CCD photometric data for one of the most complete and homogeneous samples of isolated galaxies currently available, the Catalog of Isolated Galaxies (CIG) of Karachentseva (1973). The CCD BVRI images in the Johnson-Cousins system were obtained with a SITe1 detector attached to the $1.5 \mathrm{~m}$ telescope at OAN-SPM, covering an area of about $4.3^{\prime} \times 4.3^{\prime}$, with a typical seeing of $1.8^{\prime \prime}$ and a scale of $0.51^{\prime \prime}$ pixel $^{-1}$.
The 40 galaxies reported in this work were obtained in three observing runs and no special strategy was applied in selecting this current subset. Availability of observing time and good weather conditions were the main factors constraining the number of observed galaxies. Some aspects of the selection criteria for the CIG sample that are relevant to the present and further photometric analysis are described in Hernández-Toledo et al. (1999).

\subsection{Data reduction}

A journal of the photometric observations is given in Table 1. Column (1) gives the original catalog number, and Cols. (2)-(9) give the number of frames per filter, the integration time (in seconds), and the seeing conditions (in arc-seconds).

Table 2 reports some relevant information on the observed isolated galaxies obtained from literature. Column (1) gives the CIG number, Col. (2) reports other identifications, Col. (3) gives the apparent total $m_{B}$ magnitude from the Lyon Extragalactic Database (LEDA), Col. (4) gives the Hubble Type from LEDA, Col. (5) gives the apparent total $m_{B}$ magnitude from the NASA/IPAC Extragalactic Database (NED), and Col. (6) gives the radial velocity in kilometers per second, corrected for Virgo-centric infall from LEDA.

Images were de-biased, trimmed, and flat-fielded using standard IRAF ${ }^{1}$ procedures. First, the bias level of the CCD was subtracted from all exposures.

Photometric calibration was achieved by nightly observations of standard stars of known magnitudes from the PG 1525-071, PG 1633+099, PG 1657+078, and M 67 fields of stars (Landolt 1992; and Chevalier \& Ilovaisky 1991, respectively) with a global color range $-0.20 \leq(B-V) \leq 1.13$ and $-0.40 \leq(B-I) \leq 2.27$. Once the principal extinction coefficients in $B, V, R$ and $I$ were estimated, the transformations of the instrumental magnitudes to a standard system were:

$B-b=\alpha_{B}+\beta_{B}(b-v)_{0}$,

$V-v=\alpha_{V}+\beta_{V}(b-v)_{0}$,

$R-r=\alpha_{R}+\beta_{R}(v-r)_{0}$,

$I-i=\alpha_{I}+\beta_{I}(v-r)_{0}$

where $B, V, R$ and $I$ are the standard magnitudes, $b, v, r$, and $i$ are the instrumental (and airmass-corrected) magnitudes, and $\alpha$ and $\beta$ are the transformation coefficients for each filter.

Sky background was subtracted using an interactive procedure that allows the user to select regions on the frame free of galaxies and bright stars. This was also done by modeling the whole 2-D image. Errors in the determination of the sky background are, in fact, the dominant source of errors in the estimation of total magnitudes.

Within the galaxy itself, care was taken to identify superposed stars. A final step in the basic reduction involved registration of all available frames for each galaxy and in each filter to within \pm 0.1 pixel. This step was performed by measuring centroids for foreground stars on the images and then performing geometric transformations using the geomap and geotran tasks in IRAF.

1 The IRAF package is written and supported by the IRAF programming group at the National Optical Astronomy Observatories (NOAO) in Tucson, Arizona. NOAO is operated by the Association of Universities for Research in Astronomy (AURA), Inc. under cooperative agreement with the National Science Foundation (NSF). 
Table 1. Journal of observations.

\begin{tabular}{|c|c|c|c|c|c|c|c|c|}
\hline$\overline{\overline{\text { CIG }}}$ & $\overline{\bar{B}}$ & $\overline{\langle\overline{\langle}\rangle_{\mathrm{FWHM}}}$ & $\overline{\bar{V}}$ & $\left\langle\overline{\langle V\rangle_{\mathrm{FWHM}}}\right.$ & $\overline{\bar{R}}$ & $\overline{\langle/ R\rangle_{\mathrm{FWHM}}}$ & $\bar{I}$ & $\overline{\langle\overline{\langle}}\langle\rangle_{\mathrm{FWHM}}$ \\
\hline CIG 72 & $1 \times 1200$ & 1.8 & $1 \times 600$ & 1.6 & $1 \times 300$ & 1.5 & $1 \times 300$ & 1.6 \\
\hline CIG 89 & $3 \times 900$ & 2.4 & $3 \times 240$ & 2.4 & $3 \times 120$ & 2.4 & $3 \times 90$ & 2.3 \\
\hline CIG 103 & $4 \times 600$ & 2.5 & $1 \times 600$ & 2.3 & $2 \times 300$ & 2.4 & $1 \times 300$ & 2.3 \\
\hline CIG 109 & $1 \times 1200$ & 1.8 & $1 \times 600$ & 1.8 & $1 \times 300$ & 1.6 & $1 \times 300$ & 1.5 \\
\hline CIG 135 & $1 \times 1200$ & 2.6 & $1 \times 600$ & 2.5 & $2 \times 600$ & 2.5 & $1 \times 300$ & 2.1 \\
\hline CIG 145 & $1 \times 1200$ & 1.7 & $1 \times 900$ & 1.7 & $1 \times 420$ & 1.6 & $1 \times 300$ & 1.6 \\
\hline CIG 156 & $2 \times 600$ & 1.9 & $2 \times 300$ & 1.7 & $2 \times 180$ & 1.7 & $2 \times 120$ & 1.6 \\
\hline CIG 191 & $1 \times 1200$ & 2.2 & $1 \times 600$ & 2.1 & $1 \times 300$ & 2.0 & $2 \times 180$ & 1.8 \\
\hline CIG 199 & $2 \times 1200$ & 1.9 & $2 \times 600$ & 1.8 & $2 \times 300$ & 1.7 & $2 \times 300$ & 1.6 \\
\hline CIG 309 & $1 \times 1500$ & 2.6 & $1 \times 300$ & 2.3 & $1 \times 120$ & 2.6 & $1 \times 120$ & 2.8 \\
\hline CIG 314 & $2 \times 1500$ & 2.4 & $1 \times 600$ & 2.3 & $1 \times 420$ & 2.5 & $1 \times 420$ & 2.1 \\
\hline CIG 392 & $1 \times 1200$ & 1.8 & $1 \times 600$ & 1.8 & $1 \times 300$ & 1.7 & $2 \times 180$ & 1.5 \\
\hline CIG 448 & $1 \times 1200$ & 2.1 & $1 \times 600$ & 2.2 & $1 \times 300$ & 1.9 & $2 \times 180$ & 1.9 \\
\hline CIG 477 & $1 \times 1200$ & 2.1 & $2 \times 480$ & 2.0 & $1 \times 150$ & 1.9 & $2 \times 60$ & 1.8 \\
\hline CIG 491 & $1 \times 1200$ & 1.5 & $1 \times 900$ & 1.6 & $1 \times 300$ & 1.5 & $1 \times 300$ & 1.5 \\
\hline CIG 500 & $1 \times 1200$ & 2.1 & $1 \times 900$ & 2.1 & $1 \times 300$ & 2.1 & $1 \times 300$ & 2.2 \\
\hline CIG 507 & $2 \times 1200$ & 2.1 & $2 \times 600$ & 2.1 & $2 \times 300$ & 2.0 & $2 \times 300$ & 1.9 \\
\hline CIG 512 & $2 \times 1200$ & 2.3 & $1 \times 900$ & 2.1 & $1 \times 600$ & 2.2 & $1 \times 600$ & 2.1 \\
\hline CIG 525 & $1 \times 1200$ & 2.0 & $2 \times 600$ & 1.9 & $2 \times 300$ & 1.8 & $2 \times 300$ & 1.7 \\
\hline CIG 539 & $1 \times 1200$ & 2.0 & $1 \times 600$ & 2.1 & $1 \times 300$ & 2.0 & $1 \times 300$ & 2.2 \\
\hline CIG 547 & $2 \times 1200$ & 2.4 & $1 \times 900$ & 1.9 & $1 \times 600$ & 1.9 & $1 \times 600$ & 1.8 \\
\hline CIG 575 & $1 \times 1200$ & 2.1 & $1 \times 600$ & 2.0 & $1 \times 300$ & 2.0 & $1 \times 300$ & 1.7 \\
\hline CIG 616 & $1 \times 1200$ & 2.0 & $2 \times 600$ & 2.0 & $1 \times 480$ & 2.0 & $1 \times 300$ & 1.8 \\
\hline CIG 624 & $1 \times 900$ & 1.5 & $1 \times 600$ & 1.8 & $1 \times 300$ & 1.7 & $1 \times 300$ & 1.8 \\
\hline CIG 630 & $1 \times 1200$ & 1.8 & $1 \times 900$ & 1.7 & $1 \times 300$ & 1.7 & $1 \times 300$ & 1.5 \\
\hline CIG 631 & $1 \times 1200$ & 1.7 & $1 \times 600$ & 1.6 & $1 \times 300$ & 1.6 & $1 \times 300$ & 1.5 \\
\hline CIG 712 & $1 \times 1200$ & 1.7 & $3 \times 600$ & 1.6 & $2 \times 150$ & 1.6 & $1 \times 150$ & 1.6 \\
\hline CIG 716 & $1 \times 1200$ & 2.4 & $1 \times 600$ & 2.2 & $2 \times 150$ & 2.1 & $2 \times 150$ & 1.9 \\
\hline CIG 744 & $1 \times 1200$ & 2.4 & $2 \times 600$ & 2.4 & $2 \times 300$ & 2.3 & $2 \times 600$ & 1.9 \\
\hline CIG 766 & $1 \times 1200$ & 2.3 & $1 \times 600$ & 2.3 & $1 \times 300$ & 2.0 & $1 \times 300$ & 1.7 \\
\hline CIG 772 & $1 \times 1200$ & 2.1 & $1 \times 600$ & 1.9 & $1 \times 300$ & 1.9 & $1 \times 300$ & 1.7 \\
\hline CIG 800 & $1 \times 1200$ & 1.9 & $1 \times 900$ & 1.7 & $1 \times 300$ & 1.8 & $1 \times 300$ & 1.7 \\
\hline CIG 805 & $1 \times 1200$ & 2.1 & $1 \times 900$ & 2.3 & $1 \times 300$ & 2.3 & $1 \times 300$ & 2.0 \\
\hline CIG 812 & $1 \times 1200$ & 2.3 & $1 \times 600$ & 2.4 & $1 \times 300$ & 2.5 & $1 \times 300$ & 2.3 \\
\hline CIG 838 & $1 \times 1200$ & 1.6 & $1 \times 600$ & 1.4 & $1 \times 300$ & 1.4 & $1 \times 300$ & 1.4 \\
\hline CIG 840 & $1 \times 1200$ & 1.8 & $2 \times 300$ & 2.0 & $2 \times 150$ & 1.9 & $2 \times 150$ & 1.6 \\
\hline CIG 858 & $1 \times 1200$ & 1.9 & $1 \times 600$ & 1.8 & $1 \times 480$ & 1.8 & $1 \times 420$ & 1.6 \\
\hline CIG 861 & $1 \times 1200$ & 2.2 & $1 \times 600$ & 2.2 & $1 \times 300$ & 2.1 & $1 \times 300$ & 1.9 \\
\hline CIG 862 & $1 \times 1200$ & 1.8 & $1 \times 600$ & 1.7 & $1 \times 300$ & 1.6 & $1 \times 300$ & 1.5 \\
\hline CIG 947 & $1 \times 2400$ & 1.7 & $1 \times 1500$ & 1.7 & $1 \times 600$ & 1.8 & $1 \times 300$ & 2.4 \\
\hline
\end{tabular}

\subsection{Errors}

Apparent magnitudes for each galaxy were estimated in three concentric circular apertures. This was achieved in the $B V R I$ bands by using the phot routines in IRAF. Here we report the total apparent magnitudes at the larger aperture, while in Appendix A we report apparent magnitudes at two other circular apertures, also in the BVRI bands (see Table A.1 in Appendix A).

An estimation of the errors in our photometry involves two parts: (1) the procedures to obtain instrumental magnitudes, and (2) the uncertainty when such instrumental magnitudes are transformed to the standard system.

For item 1, our estimate of the errors is mainly concerned with extinction corrections and the airmass terms. After a least-squares fit, the associated errors of the slope for each principal extinction coefficient are $\delta\left(k_{B}\right) \sim 0.031, \delta\left(k_{V}\right) \sim$ $0.022, \delta\left(k_{R}\right) \sim 0.025$ and $\delta\left(k_{I}\right) \sim 0.034$. An additional error $\delta$ (airmass) $\sim 0.01$ from the airmass routines in IRAF was also considered. For item 2, the zero point and first-order color terms are the most important to consider. The errors from the assumed relations for $\alpha$ and $\beta$ were, 0.03, 0.03, 0.02, 0.03 and 0.02, 0.02, $0.04,0.04$ in $B, V, R$ and $I$, respectively.
To estimate the total error in each band, it is necessary to propagate the above quoted errors according to the corresponding transformation equations. An additional estimate of the sky contribution is also necessary. This was achieved by estimating local and more general 2-D sky values on the images. Typical values $\delta(B) \sim 0.10, \delta(V) \sim 0.10, \delta(R) \sim 0.08$, and $\delta(I) \sim 0.10$ are obtained. Total typical uncertainties are $0.14,0.12,0.11$, and 0.16 in the $B, V, R$, and $I$ bands, respectively.

\subsubsection{Isolated galaxies}

Figure 1 shows a comparison of the estimated apparent magnitudes for the isolated galaxies in the $B, V, R$ and $I$ bands versus the corresponding values from similar aperture photometry when available in the HyperLeda database. An interpolation between adjacent aperture values was estimated when necessary.

We find a reasonable agreement with the available magnitudes from the literature, except for CIG 624, as shown in Fig. 1. We notice that the apparent diameter of this galaxy is larger than the CCD image size, difficulting a reliable estimate of the sky, thus causing an overestimation of the total magnitude at a similar aperture. A least square fit to the data in Fig. 1 shows rms 
Table 2. General data for the observed isolated spiral galaxies.

\begin{tabular}{|c|c|c|c|c|c|}
\hline CIG & ID & $m_{B}$ mag (LEDA) & Type (LEDA) & $m_{B}$ mag (NED) & $V_{\text {Rad }}\left(\mathrm{km} \mathrm{s}^{-1}\right)$ \\
\hline CIG 72 & UGC 01395 & 14.337 & $\mathrm{Sb}$ & 14.18 & 5150.9 \\
\hline CIG 89 & NGC 0821 & 11.753 & $\mathrm{E}$ & 11.67 & 1747.6 \\
\hline CIG 103 & NGC 0918 & 13.071 & $\mathrm{Sc}$ & 13.05 & 1536.2 \\
\hline CIG 109 & NGC 0949 & 12.481 & $\mathrm{Sb}$ & 12.40 & 732.7 \\
\hline CIG 135 & NGC 1474 & 14.842 & $\mathrm{Sa}$ & 14.66 & 5198.5 \\
\hline CIG 145 & NGC 1542 & 14.858 & Sab & 14.83 & 3693.5 \\
\hline CIG 156 & UGC 03258 & 13.733 & SBab & 13.98 & 2744 \\
\hline CIG 191 & UGC 03863 & 14.202 & $\mathrm{SBa}$ & 14.01 & 6084.7 \\
\hline CIG 199 & UGC 03944 & 14.968 & $\mathrm{Sc}$ & 14.60 & 4016.8 \\
\hline CIG 309 & NGC 2775 & 11.143 & $\mathrm{Sab}$ & 11.03 & 1346.3 \\
\hline CIG 314 & NGC 2776 & 12.204 & $\mathrm{SABc}$ & 12.14 & 2797.3 \\
\hline CIG 392 & PGC 029147 & 14.755 & $\mathrm{Sbc}$ & 14.99 & 7612.4 \\
\hline CIG 448 & NGC 3437 & 12.532 & $\mathrm{SABc}$ & 12.75 & 1379.7 \\
\hline CIG 477 & NGC 3655 & 12.317 & $\mathrm{Sc}$ & 12.30 & 1525.4 \\
\hline CIG 491 & UGC 06608 & 14.318 & $\mathrm{SABa}$ & 14.23 & 6246.2 \\
\hline CIG 500 & UGC 06771 & 14.452 & Sab & 13.60 & 6001.5 \\
\hline CIG 507 & UGC 06847 & 14.988 & $\mathrm{Sbc}$ & 14.97 & 5072.6 \\
\hline CIG 512 & UGC 06903 & 13.929 & $\mathrm{Sc}$ & 13.00 & 1915.8 \\
\hline CIG 525 & UGC 07416 & 13.791 & $\mathrm{Sb}$ & 13.78 & 7106.9 \\
\hline CIG 539 & NGC 4566 & 13.962 & $\mathrm{Sbc}$ & 13.87 & 5590.4 \\
\hline CIG 547 & NGC 4635 & 13.271 & $\mathrm{SABc}$ & 13.20 & 1083.9 \\
\hline CIG 575 & NGC 5016 & 13.37 & $\mathrm{SABb}$ & 13.49 & 2761.1 \\
\hline CIG 616 & UGC 09088 & 14.445 & $\mathrm{SO}-\mathrm{a}$ & 14.40 & 6452.5 \\
\hline CIG 624 & NGC 5585 & 11.391 & $\mathrm{SABc}$ & 11.20 & 573.2 \\
\hline CIG 630 & NGC 5622 & 13.981 & $\mathrm{Sb}$ & 13.99 & 4122.6 \\
\hline CIG 631 & NGC 5633 & 13.109 & $\mathrm{Sb}$ & 13.06 & 2581 \\
\hline CIG 712 & NGC 6012 & 12.871 & SBab & 12.96 & 2004.2 \\
\hline CIG 716 & UGC 10104 & 14.336 & $\mathrm{Sc}$ & 14.26 & 10056.2 \\
\hline CIG 744 & UGC 10437 & 15.107 & $\mathrm{Sc}$ & 14.70 & 2854.1 \\
\hline CIG 766 & NGC 6207 & 11.857 & $\mathrm{Sc}$ & 12.16 & 1090.3 \\
\hline CIG 772 & IC 1231 & 13.692 & $\mathrm{Sc}$ & 13.59 & 5538.9 \\
\hline CIG 800 & NGC 6347 & 14.404 & $\mathrm{SBb}$ & 14.47 & 6307.9 \\
\hline CIG 805 & IC 1256 & 14.189 & $\mathrm{Sb}$ & 14.00 & 4937.8 \\
\hline CIG 812 & NGC 6389 & 12.839 & $\mathrm{Sbc}$ & 12.82 & 3281.5 \\
\hline CIG 838 & IC 1269 & 13.428 & $\mathrm{Sbc}$ & 13.40 & 6296 \\
\hline CIG 840 & UGC 11058 & 13.476 & $\mathrm{Sb}$ & 13.29 & 4977.6 \\
\hline CIG 858 & NGC 6695 & 14.314 & $\mathrm{SBb}$ & 14.28 & 5734.7 \\
\hline CIG 861 & UGC 11352 & 15.324 & $\mathrm{SBbc}$ & 15.29 & 6894.1 \\
\hline CIG 862 & NGC 6711 & 13.715 & $\mathrm{Sbc}$ & 13.70 & 4929.6 \\
\hline CIG 947 & NGC 7217 & 11.033 & $\mathrm{Sab}$ & 11.02 & 1118.2 \\
\hline
\end{tabular}

errors of $0.13,0.15,0.06$ and 0.07 mag in the $B, V, R$ and $I$ bands respectively, in close agreement with our total errors above estimated. Additional magnitudes at two other concentric circular apertures in $B, V, R$, and $I$ for all the isolated galaxies in this study are reported in the Appendix A. A logarithmic aperture size in $0.1^{\prime}$ units was adopted according to the HyperLeda convention.

\section{Magnitudes and colors}

The estimated apparent magnitudes and the colors of the galaxies in the sample are presented in Table 3. Entries are as follows: Col. (1) gives the CIG number, Col. (2) gives the logarithmic aperture size in $0.1^{\prime}$ units as defined in HyperLeda, and Cols. (3)-(6) give the observed integrated apparent magnitudes in the $B, V, R$, and $I$ bands. Finally, Cols. (7)-(9) give the observed $(B-V),(B-R)$, and $(B-I)$ color indices.

The fraction of dust seems to be higher for bigger galaxies, according to empirical (e.g., Giovanelli et al. 1995;
Wang \& Heckman 1996; Tully et al. 1998) and theoretical (e.g., Shustov et al. 1997) arguments. Therefore, the internal extinction correction should depend not only on inclination but also on galaxy scale: $A_{\lambda}^{\mathrm{i}}[\mathrm{mag}]=\gamma_{\lambda} \log (a / b)$, where $a / b$ is the majorto-minor axis ratio, and $\gamma_{\lambda}$ is a scale-dependent coefficient in the given passband $\lambda$. From an empirical analysis, Tully et al. (1998) inferred the coefficients $\gamma_{\lambda}$ in the BRIK bands as a function of the galaxy maximum circular velocity. From their data (given in Tully \& Pierce 2000) we have carried out linear correlations of these coefficients with the corresponding magnitudes not corrected for internal extinction:

$\gamma_{B}[\mathrm{mag}]=-6.30-0.40 M_{B}, \quad M_{B}<-16.7$, $\gamma_{R}[\mathrm{mag}]=-4.20-0.26 M_{B}, \quad M_{R}<-17.7$, $\gamma_{I}[\mathrm{mag}]=-3.40-0.20 M_{I}, \quad M_{I}<-18.0$,

$\gamma_{K}[\mathrm{mag}]=-0.85-0.05 M_{K}, \quad M_{K}<-19.7$.

For values of the magnitudes higher than the limits given in Eq. (2), $\gamma_{\lambda}$ is assumed to be 0 (no extinction correction). For band $V$, the line coefficients of $\gamma_{V}$ were obtained by a simple 

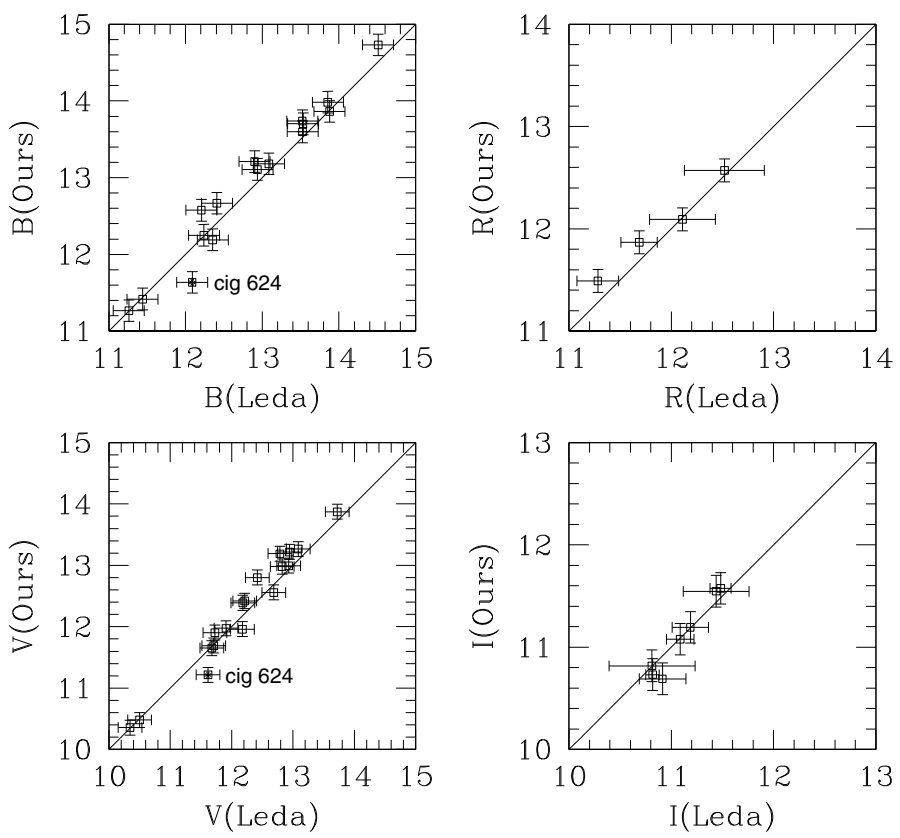

Fig. 1. Comparison between our total $B, V, R$ and $I$ apparent magnitudes and the available photometry of similar aperture from the HyperLeda Database. A discrepant case (CIG 624) is indicated.

interpolation of those in bands $B, R, I$, and $K: \gamma_{V}[\mathrm{mag}]=$ $-4.67-0.29 M_{V}, M_{V}<-17.5$. The $a / b$ ratios estimated at the $B$-band $25 \mathrm{mag} / \operatorname{arcsec}^{2}$ isophote were taken from the HyperLeda database.

Table 4 shows foreground - and internal - extinctioncorrected color indices and absolute magnitudes. Corrections are based on data generated from the dust galaxy maps given in Schlegel et al. (1998) and available in NED. Entries are as follows: Col. (1) gives the identification CIG number, Cols. (2)-(4) give the corrected $(B-V),(B-R)$, and $(B-I)$ color indices. Finally, Cols. (5)-(8) report the corrected absolute magnitudes in the $B, V, R$, and $I$ bands. A Hubble constant value of $70 \mathrm{~km} \mathrm{~s}^{-1} \mathrm{Mpc}^{-1}$ was adopted.

The $(B-V)$-corrected colors span the range of $0.15-1.0 \mathrm{mag}$, comparable to that reported in de Jong (1996), Verheijen (1997) and more recently Hernández-Toledo et al. (2007). A more physical correction applied to the luminosities yields a $B$-band luminosity range $\left(-17.5 \leq M_{B} \leq-22.05\right)$, indicating no faint spirals in this sample, except perhaps for the case of CIG 547.

In Fig. 2 we plot different extinction-corrected colormagnitude diagrams $\left(M_{R}\right.$ vs. $(B-V),(B-R)$, and $(B-$ $I)$ colors) for our sample of isolated galaxies. We find a loose correlation of colors with magnitude. Some authors (e.g., Avila-Reese \& Firmani 2000) have stated that a significant dependence of color on luminosity for normal isolated disks is not expected.

\section{Optical and near-infrared (NIR) morphology}

To discuss the optical morphology we present an image processing scheme that enhances high/low surface brightness morphological components in galaxies. For each isolated galaxy, a mosaic like the one shown in Fig. 3 is presented. Each mosaic includes, from top-left to bottom-right, (1) a logarithmic gray scale $R$-band image to look for both internal and external details; (2) an $R$-band filter-enhanced image to look for internal structure in the form of star-forming regions and/or structure embedded in dusty regions (the filtering-enhancing techniques Sofue 1993, allow to subtract the diffuse background in a convenient way for discussing the different morphological details); (3) a (B-I) color index map to visualize the spatial distribution of dusty and SF regions (light-gray is for blue colors, while dark-gray is for red colors); (4) a composed (filter-enhanced) NIR $J H K$ image which is a combination of the archived $J$-, $H$-, and $K$-band images from 2MASS (Skrutskie et al. 2006) to complement the structural and morphological analysis. At the far right, (5) the ellipticity $\epsilon$ and position angle (PA) radial profiles from the $R$ and composed $J H K$ images to provide evidence of the presence of some structural components like bars, rings and other features. Finally (6), an $R G B$ image from the SDSS database is included (when available) to visualize the color distribution of the different structural components in these galaxies and at the same time to have a wider view of their local environment. This is usefull to identify possible dwarf galaxies interacting with CIG objects but that are not visible in our narrow optical images. The $R G B$ images allow us either to explore the close environment of an isolated galaxy (see square symbols on the $R G B$ images indicating a few objects identified as galaxies, according to SDSS) or take a look at the spatial distribution of different stellar populations, dust lanes and SF regions, according to their color distribution. Notice that there is no radial velocity information for those objects identified as galaxies and that we do not pretend to give an exhaustive list of such objects (see Verley et al. 2007).

We identify a bar signature if the ellipticity radial profile $\epsilon$ rises to a maximum $\epsilon_{\max }$ required to be above that of the outer disk, while the PA radial profile shows a plateau (within $\pm 20^{\circ}$ ) along the bar (Wozniak et al. 1995).

All the images are oriented according to the standard (northeast) astronomical convention. The NIR images are approximately at the same scale as the optical images. For the sake of not crowding, the major diameter (in arcmin) of the optical images is specified in the caption for each galaxy. In some cases, not all the foreground stars in each field have been removed.

In the following discussion, we use the fact that the median value of the $(B-V)$ color declines systematically as the morphological type $T$ increases along the morphological sequence (Roberts \& Haynes 1994).

\subsection{Comments on individual objects}

CIG 72. The galaxy was previously classified as SA(rs)b Sy1.9 by NED. In the composed $J H K$ and the optical filter-enhanced images we find evidence of a bar structure, as well as an inner ring. The $B-V$ color index corresponds to those for $\mathrm{Sa} / \mathrm{Sab}$ types. The geometric profiles confirm the presence of a bar structure at a radius of approx. 16 arcsec. We classify this object as $\mathrm{SB}(\mathrm{r}) \mathrm{b}$. Notice the degree of global asymmetry. This object has been flagged as possibly interacting by Sulentic et al. (2006). We notice an asymmetric pattern in the arms.

CIG 89. NED reports this galaxy as E6? In the optical and NIR images we see a disk structure. The whole disk shows uniform colors in the $B-I$ color map. The total $B-V$ color index is similar to the $\mathrm{S} 0 \mathrm{a} / \mathrm{Sa}$ types. Our classification for this galaxy is $\mathrm{S} 0$.

CIG 103. According to NED this is a $\mathrm{SAB}(\mathrm{rs}) \mathrm{c}$ galaxy. The filter-enhanced images show evidence of an ovally-shaped bulge region. The $B-V$ color index is representative of the ScdSd types. The geometric profile in both the $J H K$ and $R$ bands show weak evidence of a bar in the first 10 arcsec. We classify this galaxy as $\mathrm{SAB}(\mathrm{r}) \mathrm{cd}$. 
Table 3. Apparent magnitudes and color indices.

\begin{tabular}{|c|c|c|c|c|c|c|c|c|}
\hline$\overline{\mathrm{CIG}}$ & $\overline{\log (\mathrm{A})}$ & $\overline{\bar{B}}$ & $\overline{\bar{V}}$ & $\overline{\bar{R}}$ & $\overline{\overline{I I}}$ & $\overline{B-V}$ & $\overline{B B-R}$ & $\overline{B B-I}$ \\
\hline CIG 72 & 1.181 & 14.449 & 13.619 & 13.090 & 12.381 & 0.830 & 1.359 & 2.068 \\
\hline CIG 89 & 1.385 & 12.210 & 11.310 & 10.739 & 9.744 & 0.900 & 1.470 & 2.466 \\
\hline CIG 103 & 1.497 & 13.359 & 12.440 & 11.811 & 11.078 & 0.919 & 1.548 & 2.280 \\
\hline CIG 109 & 1.376 & 12.667 & 11.975 & 11.490 & 10.790 & 0.691 & 1.177 & 1.876 \\
\hline CIG 135 & 1.223 & 14.662 & 13.762 & 13.190 & 12.419 & 0.900 & 1.472 & 2.244 \\
\hline CIG 145 & 1.284 & 15.020 & 13.886 & 13.228 & 12.363 & 1.134 & 1.792 & 2.657 \\
\hline CIG 156 & 1.102 & 13.597 & 12.967 & 12.520 & 11.812 & 0.630 & 1.078 & 1.786 \\
\hline CIG 191 & 1.223 & 14.497 & 13.481 & 13.327 & 11.922 & 1.016 & 1.170 & 2.575 \\
\hline CIG 199 & 1.327 & 14.481 & 13.889 & 13.424 & 12.761 & 0.592 & 1.057 & 1.720 \\
\hline CIG 309 & 1.403 & 11.417 & 10.481 & 9.920 & 8.994 & 0.936 & 1.497 & 2.422 \\
\hline CIG 314 & 1.591 & 12.249 & 11.693 & 11.285 & 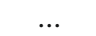 & 0.555 & 0.963 & 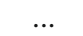 \\
\hline CIG 392 & 1.102 & 14.952 & 14.077 & 13.544 & 12.833 & 0.875 & 1.408 & 2.119 \\
\hline CIG 448 & 1.482 & 12.575 & 11.961 & 11.474 & 10.818 & 0.614 & 1.101 & 1.758 \\
\hline CIG 477 & 1.306 & 12.575 & 11.904 & 11.366 & 10.732 & 0.671 & 1.210 & 1.844 \\
\hline CIG 491 & 1.066 & 14.602 & 13.915 & 13.411 & 12.776 & 0.687 & 1.191 & 1.826 \\
\hline CIG 500 & 1.272 & 13.911 & 13.018 & 12.471 & 11.812 & 0.893 & 1.440 & 2.099 \\
\hline CIG 507 & 1.306 & 15.299 & 14.698 & 14.206 & 13.386 & 0.601 & 1.093 & 1.913 \\
\hline CIG 512 & 1.428 & 13.623 & 13.011 & 12.508 & 11.890 & 0.613 & 1.115 & 1.733 \\
\hline CIG 525 & 1.248 & 13.863 & 13.190 & 12.673 & 12.059 & 0.673 & 1.190 & 1.805 \\
\hline CIG 539 & 1.248 & 14.171 & 13.307 & 12.774 & 12.094 & 0.864 & 1.397 & 2.077 \\
\hline CIG 547 & 1.403 & 13.597 & 12.995 & 12.571 & 12.082 & 0.602 & 1.026 & 1.515 \\
\hline CIG 575 & 1.357 & 13.180 & 12.558 & 12.093 & 11.547 & 0.622 & 1.088 & 1.633 \\
\hline CIG 616 & 1.445 & 14.380 & 13.245 & 12.646 & 11.886 & 1.135 & 1.735 & 2.495 \\
\hline CIG 624 & 1.482 & 11.634 & 11.216 & 10.814 & 10.123 & 0.418 & 0.820 & 1.512 \\
\hline CIG 630 & 1.385 & 14.105 & 13.440 & 12.948 & 12.412 & 0.665 & 1.157 & 1.693 \\
\hline CIG 631 & 1.357 & 13.176 & 12.932 & 12.449 & 11.575 & 0.244 & 0.727 & 1.601 \\
\hline CIG 712 & 1.357 & 13.209 & 12.420 & 11.870 & 11.195 & 0.789 & 1.339 & 2.013 \\
\hline CIG 716 & 1.181 & 14.730 & 13.874 & 13.344 & 12.636 & 0.856 & 1.387 & 2.094 \\
\hline CIG 744 & 1.428 & 14.550 & 14.045 & 13.686 & 13.193 & 0.505 & 0.864 & 1.357 \\
\hline CIG 766 & 1.357 & 12.189 & 11.651 & 11.240 & 10.691 & 0.538 & 0.949 & 1.497 \\
\hline CIG 772 & 1.403 & 13.846 & 13.203 & 12.705 & 12.175 & 0.643 & 1.141 & 1.672 \\
\hline CIG 800 & 1.223 & 14.263 & 13.450 & 12.890 & 12.218 & 0.813 & 1.373 & 2.045 \\
\hline CIG 805 & 1.248 & 14.297 & 13.551 & 13.022 & 12.434 & 0.746 & 1.275 & 1.863 \\
\hline CIG 812 & 1.445 & 13.107 & 12.389 & 11.848 & 11.211 & 0.718 & 1.259 & 1.896 \\
\hline CIG 838 & 1.338 & 14.026 & 13.218 & 12.709 & 12.189 & 0.808 & 1.317 & 1.837 \\
\hline CIG 840 & 1.306 & 13.701 & 12.981 & 12.462 & 11.909 & 0.720 & 1.240 & 1.792 \\
\hline CIG 858 & 1.248 & 14.374 & 13.493 & 12.917 & 12.226 & 0.881 & 1.457 & 2.148 \\
\hline CIG 861 & 1.181 & 15.428 & 14.685 & 14.190 & 13.590 & 0.743 & 1.237 & 1.838 \\
\hline CIG 862 & 1.223 & 13.984 & 13.267 & 12.735 & 12.222 & 0.718 & 1.249 & 1.763 \\
\hline CIG 947 & 1.489 & 11.265 & 10.355 & $\ldots$ & $\ldots$ & 0.910 & $\cdots$ & $\ldots$ \\
\hline
\end{tabular}

CIG 109. NED classified this galaxy as SA(rs)b. The geometric profiles resemble a bar but a careful examination of our images indicate that two strong knots at opposite sides in the bulge region produce an almost constant PA. The $B-V$ color index is representative of Sbc-Sc types. The $B-I$ color map shows a central region with a mixture of dust and SF regions, and also evidence of an outer and smoother structure (arms?). We classify this object as $(\mathrm{R}) \mathrm{SA}(\mathrm{s}) \mathrm{ab}$.

CIG 135. NED classification for this galaxy is SBa. The composed $J H K$ image shows evidence of a bar and an inner ring more clearly than the optical filter-enhanced one. The outer arms are fragmented, resembling tidal disturbances. The $B-V$ color index is representative of the $\mathrm{Sab} / \mathrm{Sb}$ types. We classify this galaxy as SB(r)ab. This object has been flagged as possibly interacting by Sulentic et al. (2006).

CIG 145. According to NED this is a Sab galaxy. In spite of the high inclination, the $R$ band filter-enhanced image show a prominent dust lane and a structure that resembles an arm. The $B-V$ color index is probably reddened and is characteristic of the $\mathrm{S} 0 \mathrm{a} / \mathrm{Sa}$ types. We classify this galaxy as $\mathrm{Sa}$.

CIG 156. The NED classification for this galaxy is $\mathrm{SB}(\mathrm{r}) \mathrm{ab}$ pec. The optical and near-infrared images as well as the geometric profiles show a strong bar. The shape of the arms resembles an outer ring. The $B-I$ color map shows that in general the galaxy has blue colors, except in the nuclear region where it is reddened. The $B-V$ color is consistent with the $\mathrm{Sbc} / \mathrm{Sc}$ types. We classify this object as (R)SBbc. This object has been flagged as possibly interacting by Sulentic et al. (2006).

CIG 191. The NED classification for this object is $\left(R^{\prime}\right) S B a$. We observe a bar structure in the filter-enhanced images. The $B-V$ color index is representative of $\mathrm{S} 0 \mathrm{a} / \mathrm{Sa}$ galaxies. The geometric profiles also confirm the existence of a bar in both $R$ and $J H K$ filter-enhanced images. We classify this galaxy as (R)SBa.

CIG 199. NED reports this object as Scd. The optical and filter-enhanced images show a set of clumpy and blue arms. The $R$ filter-enhanced image as well as the geometric profiles show weak evidence of an inner bar. The $R G B$ color image shows something similar to an inner pseudo-ring and makes more evident the fragmentation of the outer arms. The squares show the position of three objects classified as galaxies in the field of CIG 199. The $B-V$ color index is representative of the $\mathrm{Sm} / \mathrm{Im}$ types. We classify this galaxy as $\mathrm{SAB}(\mathrm{r}) \mathrm{cd}$.

CIG 309. The NED classification for this galaxy is $S A(r) a b$. Tight arms with strong inner star forming regions and dust lanes 
Table 4. Corrected colors and absolute magnitudes.

\begin{tabular}{|c|c|c|c|c|c|c|c|}
\hline$\overline{\text { CIG }}$ & $\overline{(B-V)_{\mathrm{c}}}$ & $\overline{(B-R)_{\mathrm{c}}}$ & $\overline{(B-I)_{\mathrm{c}}}$ & $\overline{M_{B}}$ & $\overline{M_{V}}$ & $\overline{M_{R}}$ & $\overline{M_{I}}$ \\
\hline CIG 72 & 0.689 & 1.170 & 1.766 & -20.496 & -21.185 & -21.665 & -22.261 \\
\hline CIG 89 & 0.763 & 1.264 & 2.157 & -20.371 & -21.135 & -21.636 & -22.529 \\
\hline CIG 103 & 0.471 & 0.877 & 1.270 & -20.241 & -20.712 & -21.118 & -21.511 \\
\hline CIG 109 & 0.588 & 1.054 & 1.657 & -17.890 & -18.478 & -18.944 & -19.547 \\
\hline CIG 135 & 0.648 & 1.094 & 1.674 & -20.777 & -21.425 & -21.871 & -22.451 \\
\hline CIG 145 & 0.797 & 1.332 & 1.898 & -20.190 & -20.987 & -21.522 & -22.088 \\
\hline CIG 156 & 0.519 & 0.922 & 1.548 & -19.826 & -20.346 & -20.749 & -21.375 \\
\hline CIG 191 & 0.793 & 0.842 & 2.101 & -21.214 & -22.007 & -22.056 & -23.315 \\
\hline CIG 199 & 0.370 & 0.799 & 1.293 & -20.192 & -20.563 & -20.992 & -21.486 \\
\hline CIG 309 & 0.854 & 1.389 & 2.249 & -20.377 & -21.231 & -21.765 & -22.626 \\
\hline CIG 314 & 0.345 & 0.730 & & -21.569 & -21.914 & -22.300 & $\ldots$ \\
\hline CIG 392 & 0.820 & 1.346 & 2.008 & -20.487 & -21.307 & -21.833 & -22.495 \\
\hline CIG 448 & 0.450 & 0.941 & 1.457 & -19.576 & -20.026 & -20.518 & -21.033 \\
\hline CIG 477 & 0.593 & 1.121 & 1.688 & -19.446 & -20.038 & -20.567 & -21.134 \\
\hline CIG 491 & 38 & 1.081 & 1.635 & -20 & -21.154 & -21.648 & -22.201 \\
\hline CIG 500 & 13 & 1 . & 1.541 & -22.043 & -22.656 & -23.179 & -23.584 \\
\hline 5 & 403 & 0.900 & 1.572 & -19.807 & -20.210 & -20.707 & -21.379 \\
\hline G 512 & 568 & 1.062 & 1.643 & -18.747 & -19.315 & -19.810 & -20.390 \\
\hline CIG 525 & 86 & 1.086 & 1.635 & -21.523 & -22.109 & -22.609 & -23.158 \\
\hline CIG 539 & 0.778 & 1.298 & 1.901 & -20.732 & -21.510 & -22.029 & -22.633 \\
\hline CIG 547 & 0.554 & 0.970 & 1.410 & -17.568 & -18.122 & -18.538 & -18.978 \\
\hline CIG 575 & 0.552 & 1.008 & 1.496 & -20.094 & -20.646 & -21.102 & -21.589 \\
\hline CIG 616 & 0.985 & 1.578 & 2.168 & -21.292 & -22.277 & -22.870 & -23.461 \\
\hline CIG 624 & 0.347 & 0.750 & 1.388 & -18.191 & -18.538 & -18.940 & -19.578 \\
\hline CIG 630 & 0.540 & 1.021 & 1.449 & -20.260 & -20.800 & -21.281 & -21.709 \\
\hline CIG 631 & 0.127 & 0.604 & 1.415 & -20.047 & -20.174 & -20.651 & -21.463 \\
\hline CIG 712 & 0.710 & 1.243 & 1.847 & -19.433 & -20.143 & -20.676 & -21.280 \\
\hline CIG 716 & 0.776 & 1.280 & 1.925 & -21.405 & -22.181 & -22.685 & -23.330 \\
\hline CIG 744 & 471 & 0.825 & 38 & -18.637 & -19.108 & -19.462 & -19.925 \\
\hline CIG 766 & & & 56 & -19 & -19.689 & -20.099 & -20.537 \\
\hline 772 & & & 27 & -21 & -21 & -22.326 & -22.707 \\
\hline 800 & & 1.097 & 1.591 & -21 . & -22.050 & -22.557 & -23.051 \\
\hline 805 & & & 1.589 & -20 & -21.128 & -21.625 & -22.103 \\
\hline CIG 812 & 9 & 1.015 & 1.516 & -20.991 & -21.530 & -22.006 & -22.507 \\
\hline CIG 838 & 0.631 & 1.076 & 1.453 & -21.499 & -22.130 & -22.575 & -22.952 \\
\hline CIG 840 & 4 & 1.090 & 1.552 & -21.043 & -21.647 & -22.134 & -22.595 \\
\hline G 858 & 0.716 & 1.245 & 1.798 & -20.925 & -21.641 & -22.171 & -22.723 \\
\hline CIG 861 & 0.543 & 0.993 & 1.425 & -20.392 & -20.935 & -21.386 & -21.817 \\
\hline CIG 862 & 0.616 & 1.097 & 1.533 & -20.686 & -21.301 & -21.783 & -22.219 \\
\hline CIG 947 & 0.796 & $\ldots$ & $\ldots$ & -20.251 & -21.048 & $\ldots$ & $\cdots$ \\
\hline
\end{tabular}

can be appreciated forming a ring that encloses a uniformly redder and soft bulge. Our optical images as well as the $R G B$ color image show how the spatial distribution of the inner star forming regions and dust lanes trace the arms. A few small galaxies (square symbols) can be appreciated in the neighborhood of this galaxy. The total $B-V$ color index is representative of the E/S0 types. We classify this galaxy as SA(r)a.

CIG 314. NED classifies this galaxy as SAB(rs)c. A set of multiple fragmented arms with star formation and blue colors can be seen in our optical images as well as in the color image from SDSS, while the central region appears redder. The composed $J H K$ image shows an inner ring, and this is also appreciated from the corresponding geometric profiles. A few dwarf galaxies (square symbols) are indicated in the $R G B$ image. The total $B-V$ color index is similar to the $\mathrm{Sm} / \mathrm{Im}$ types. Our classification for this galaxy is $\mathrm{SA}(\mathrm{r}) \mathrm{d}$.

CIG 392. NED classifies this galaxy as S0. The logarithmic, filter-enhanced $R$ band and $R G B$ images show a pair of tightly wounded arms, an elongated central region and an inner ring that appears blue in the $B-I$ color map and the $R G B$ image. The composed $J H K$ image gives strong evidence about the bar and the ring, confirmed by the geometric profiles. The total
$B-V$ color index is representative of the $\mathrm{S} 0 \mathrm{a} / \mathrm{Sa}$ types. We classify this galaxy as $\mathrm{SB}(\mathrm{r})$ a. Notice a filamentary structure coming out of the ring in the southern part.

CIG 448. The NED classification for this object is $\mathrm{SAB}(\mathrm{rs}) \mathrm{c}$. This inclined galaxy shows a set of multiple and clumpy arms that appear blue in both the $B-I$ color map and the $R G B$ image. These images also allow to appreciate dust lanes along the disk. Notice how the bulge region is emphasized in the $R G B$ and $J H K$ images. Contrary to the optical images, the $J H K$ image shows two main arms and weak evidence of a bar, instead. A few dim galaxies are appreciated in the neighborhood. The total $B-V$ color index is intermediate between the $\mathrm{Scd} / \mathrm{Sd}$ and the $\mathrm{Sm} / \mathrm{Im}$ types. We classify this galaxy as SABc.

CIG 477. SA(s)c HII is the classification reported by NED. While our optical and the $R G B$ images show a multiple and fragmented set of arms with a small bulge, the near-infrared image describes three arms and a slightly more prominent bulge. A field star near the center is masking some details inside that region. There is a faint dwarf galaxy in the field, from the SDSS image. The total $B-V$ color index is intermediate between $\mathrm{Sab} / \mathrm{Sb}$ and $\mathrm{Sbc} / \mathrm{Sc}$ types. We classify this galaxy as Sc. 


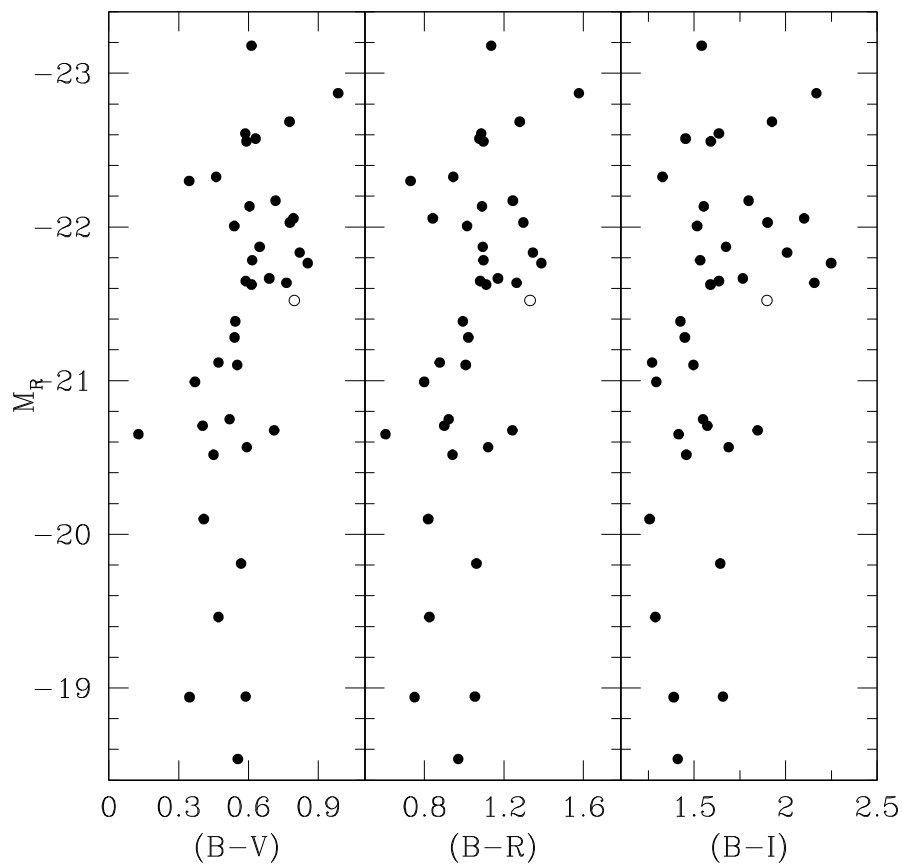

Fig. 2. Color-magnitude diagrams for the 40 isolated spirals after galactic and internal extinction corrections. Galaxies with inclination higher than $80^{\circ}$ are shown with (open) circles.

CIG 491. The NED classification for this galaxy is $\mathrm{SAB}(\mathrm{r}) \mathrm{ab}$ ? This galaxy shows a set of multiple and fragmented arms as well as an apparent inner ring, as seen in the $R$ filterenhanced image. Although the $R$ filter-enhanced image seems to show an ovally-distorted central region, neither the composed $J H K$ image nor its corresponding geometric profile confirm this. There is no information in the image from SDSS about faint galaxies close to CIG 491. The total $B-V$ color is similar to the $\mathrm{Sbc} / \mathrm{Sc}$ types. $\mathrm{SABc}(\mathrm{r})$ is our classification for this object.

CIG 500. NED reports this galaxy as (R')SAB(r)ab. A welldefined bar encircled by a ring structure is shown in the optical, $J H K$ and $R G B$ images. The arms appear to emerge from the opposite sides of the bar. From the $R G B$ image, a few dwarf galaxies can be seen projected along the face of this galaxy. The total $B-V$ color index corresponds to the $\mathrm{Sa} / \mathrm{Sab}$ types. We classify this galaxy as $\mathrm{SB}(\mathrm{r}) \mathrm{b}$.

CIG 507. NED reports this galaxy as Sbc. Although this is a highly inclined galaxy, the optical and $R G B$ images show blue, fragmented and clumpy arms and a non-prominent bulge. The composed $J H K$ image shows an elongated central region, and the geometric profiles resemble a large-scale bar-like structure. If not due to projection effects, this may be suggesting the presence of a bar. There are several dwarf galaxies identified in the image from SDSS. The total $B-V$ color index is similar to the $\mathrm{Sm} / \mathrm{Im}$ types. We classify this galaxy as SABcd. This object has been flagged as possibly interacting by Sulentic et al. (2006).

CIG 512. According to NED this is a SB(s)cd galaxy. A pair of arms emerge from opposite sides of a bar. These arms become fragmented and clumpy in the outer regions, as can be seen in the optical images and more clearly in the image from SDSS. Notice the blue color of the bar in the $B-I$ color map and the $R G B$ image. The image from SDSS also shows some faint galaxies. The total $B-V$ color index is characteristic of the Sbc/Sc types. SBcd is our classification for this galaxy.

CIG 525. NED reports this object as $\mathrm{SB}(\mathrm{r}) \mathrm{b}$. A symmetric multi-arm spiral pattern is seen emerging from opposite sides of a bar. Both the ring and the bar are confirmed by the geometric profiles, the $J H K$ and the $R G B$ images. We also notice two dwarf galaxy candidates at the end of the north-east set of arms in the optical images, but the image from SDSS only identifies one. The total $B-V$ color index is similar to the $\mathrm{Sbc/Sc}$ types. Our classification for this galaxy is $\mathrm{SB}(\mathrm{r}) \mathrm{bc}$.

CIG 539. NED reports this object as Sb. A set of two tightly wounded arms forming a ring are appreciated in all of the images. In contrast to the $R G B$ image, the diffuse outskirts are only seen in our optical images, illustrating their deeper nature. The $R$-band filter-enhanced and $J H K$ images show clear evidence of a slightly twisted bar. The $R G B$ image shows 3 dwarf galaxies in the field. The total $B-V$ color index is characteristic of the $\mathrm{S} 0 \mathrm{a} / \mathrm{Sa}$ types. We classify this galaxy as SB(r)ab. This object has been flagged as possibly interacting by Sulentic et al. (2006).

CIG 547. The NED classification for this galaxy is $\mathrm{SAB}(\mathrm{s}) \mathrm{d}$. Two adjacent bright knots near the central region may give the appearance of an ovally-distorted region. However, from our images and profiles there is no evidence of a bar-like structure. Both the $R$ band filter-enhanced and the $R G B$ image shows a pattern of blue fragmented clumpy arms and strong dust lane structures. A couple of red faint galaxies are seen in the SDSS image at the south-west set of arms. The total $B-V$ color index is characteristic of the $\mathrm{Sbc/Sc}$ morphological types. We classify this galaxy as $\mathrm{Sd}$.

CIG 575. This galaxy is reported by NED as SAB(rs)c SBNG. The optical images show a set of fragmented arms and only weak evidence of a bar. The geometric profiles and the $J H K$ image confirm this, and the $R G B$ image shows strong dust lane structures. From this image, there is no information about possible dwarf galaxies. The total $B-V$ color index is representative of the $\mathrm{Sbc/Sc}$ types. Our classification for this object is SABc.

CIG 616. NED reports this object as S0/a. This is a highly inclined galaxy. However the optical images show weak evidence of outer spiral blue arms as judged by the filter-enhanced and $B-I$ images, and a chain of SF regions at the south-west side. The optical filter-enhanced and composed JHK images show an inner region that resembles either a spiral pattern or a bar structure. There are a couple dim galaxies shown in the $R G B$ image. The total $B-V$ color index is similar to the E/S0 types. We classify this galaxy as SABab.

CIG 624. $\mathrm{SAB}(\mathrm{s}) \mathrm{d} \mathrm{HII}$ is the NED classification for this galaxy. A fragmented spiral arm pattern and regions of star formation all along the disk are appreciated both in our optical and $R G B$ images. The total $B-V$ color index is similar to the $\mathrm{Sm} / \mathrm{Im}$ types. SABdm is our classification for this galaxy. Note that the angular scale of this galaxy is larger than our CCD size (5.8 arcmin). However a wider view from the SDSS image allows us to barely identify the global spiral and fragmented pattern, and a few dwarf galaxies (green boxes) at the outskirts.

CIG 630. The NED classification for this object is Sb. Both the $R$ band filter-enhanced and the $R G B$ images show two welldefined symmetric arms spiraling twice around the central region. There is no evidence of a bar. Two faint galaxies are identified in the image from SDSS, one of them apparently over an arm of CIG 630 . The $B-V$ total color index is representative of the $\mathrm{Sbc} / \mathrm{Sc}$ types. We classify this galaxy as $\mathrm{SA}(\mathrm{rs}) \mathrm{bc}$.

CIG 631. (R)SA(rs)b is the NED classification for this object. A couple of tight arms emerge from the central region forming an apparent ring. These arms appear fragmented in the inner region and become smoother towards the external parts. The apparent ring is clearly seen in the $R G B$ and composed $J H K$ images, and also confirmed by the geometric profiles. The image 

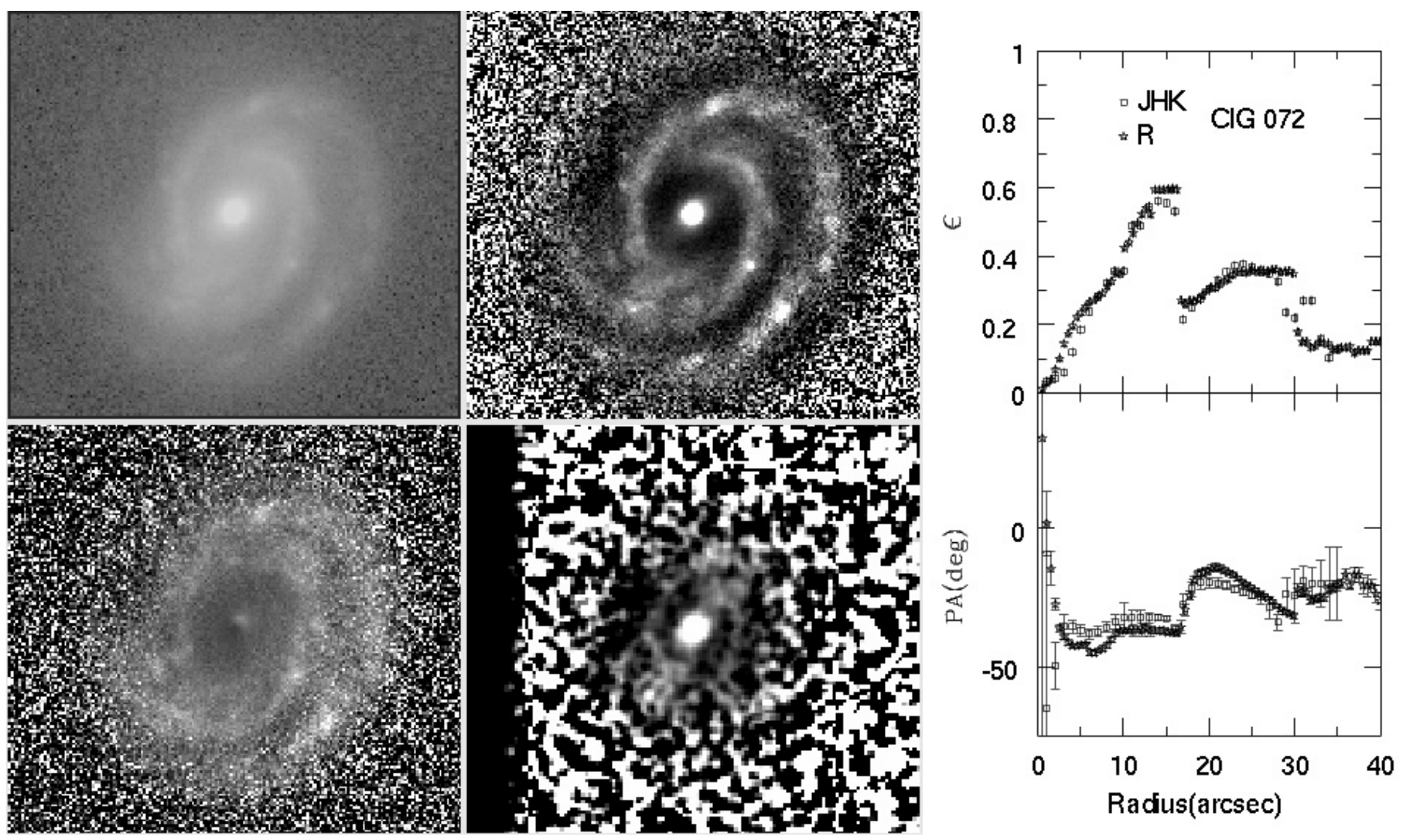

Fig. 3. CIG 72 Mosaic. Upper-left: a gray scale $R$-band image displayed in logarithmic scale. Upper-right: an $R$ band filter-enhanced image. Lowerleft: a $(B-I)$ color index map. Lower-right: a composed (filter-enhanced) NIR JHK image. Right-most panel: the photometric $\epsilon$ and PA radial profiles from the $R$-band and composed $J H K$-band images. Images are oriented according to the astronomical convention. The major diameter of the galaxy in the optical images is $1.3 \mathrm{arcmin}$.

from SDSS gives no information about possible dwarf galaxies. The total $B-V$ color index is similar to the $\mathrm{Sm} / \mathrm{Im}$ types. We classify this galaxy as SA(r)bc.

CIG 712. This object is reported as (R)SB(r)ab: LINER by NED. The optical, $R G B$ and near-infrared images show a strongly elongated inner ring along the bar and another roundshaped ring encircling the first. The geometric profiles confirm a large-scale bar and the rings. The inner ring shows blue $B-I$ colors. The optical images show evidence of a diffuse outer set of arms. The $R G B$ image shows 5 galaxies in the field, and one of them, flagged with a "B", superposed on a bright star. The magnitudes in the SDSS ugriz system for "B" are 15.27, 13.76, $12.16,14.80$ and 13.68 , respectively. The total $B-V$ color index is consistent with $\mathrm{S} 0 \mathrm{a} / \mathrm{Sa}$ and $\mathrm{Sab} / \mathrm{Sb}$ types. We classify this galaxy as $\mathrm{SB}(\mathrm{r}) \mathrm{b}$.

CIG 716. NED reports this object as $\mathrm{SA}(\mathrm{rs}) \mathrm{bc}$. Two symmetric and continuous arms emerge from an inner ring. However this pattern becomes fragmented and strongly asymmetric in the outer regions. The absence of a clear underlying disk and their morphology, suggests a possible tidal origin of these pseudoarms. Five dwarf galaxies can be appreciated in the image from SDSS. The total $B-V$ color index is characteristic of the $\mathrm{S} 0 \mathrm{a} / \mathrm{Sa}$ types. We classify this galaxy as SA(r)b.

CIG 744. NED reports this galaxy as Sc. Our optical and $R G B$ images allow us to appreciate a set of 3 arms emerging from an inner ring that encloses a faint bulge, barely visible in the image from SDSS. Notice some filamentaty structurew emerging perpendicular to the north-west arm. There is no evidence of a bar either in the images or the geometric profiles. The total $B-V$ color index is representative of the $\mathrm{Scd} / \mathrm{Sd}$ types.
The image from SDSS shows several dwarf galaxies. We classify this galaxy as Sd. This object has been flagged as possibly interacting by Sulentic et al. (2006).

CIG 766. The NED classification for this galaxy is SA(s)c HII. A pattern of multiple, fragmented and dusty structures along the arms are shown above an underlying smooth disk in our optical and $R G B$ images. In contrast, the near-infrared image shows evidence of a single inner spiral pattern that probably bifurcates outwards and also suggests for a elongated central region. A star close to the central region is masking some morphological details, as seen in the optical and $R G B$ images, where 5 dwarf galaxies can be appreciated. The north-eastern arms appear brighter than their south-western contraparts. The total $B-V$ color index is characteristic of the $\mathrm{Sm} / \mathrm{Im}$ types. SABcd is our classification for this galaxy.

CIG 772. NED reports this galaxy as Scd. A set of multiple arms that become fragmented in the outer regions can be seen in the optical images. The $R$ band filter-enhanced image shows an s-shaped central region. We find no evidence of a bar in any of the images, and the geometric profiles confirm this. The total $B-V$ color index is similar to the $\mathrm{Scd} / \mathrm{Sd}$ types. We classify this galaxy as $\mathrm{SA}(\mathrm{s}) \mathrm{cd}$.

CIG 800. $\mathrm{SBb}$ is the NED classification for this galaxy. The optical images show two main blue arms that bifurcate outwards, with an s-shaped central region. The composed JHK image shows the main arms and a elongated central region suggested as a bar by the geometric profiles. The total $B-V$ color index corresponds to the $\mathrm{Sab} / \mathrm{Sb}$ or $\mathrm{Sbc} / \mathrm{Sc}$ types. We classify this galaxy as a $\mathrm{SB}(\mathrm{s}) \mathrm{bc}$. 
CIG 805. According to NED this is an Sb galaxy. An inner ring and two arms that bifurcate and become diffuse outwards are appreciated in the optical and $R G B$ images. The composed $J H K$ image reveals what might be interpreted as an ovally distorted central region, also seen in the $R G B$ image, but there is not clear evidence of a bar in the geometric profiles. The image from SDSS shows some dwarf galaxies in the field. The total $B-V$ color index is representative of the $\mathrm{Sab} / \mathrm{Sb}$ types. Our classification for this galaxy is $\mathrm{SAB}(\mathrm{rs}) \mathrm{bc}$.

CIG 812. NED classifies this galaxy as Sbc. A set of multiple and fragmented arms that appear blue in the $B-I$ color map, are appreciated. An inner ring, confirmed in the geometric profiles, is also appreciated. The total $B-V$ color index is characteristic of the $\mathrm{Sbc} / \mathrm{Sc}$ types. We classify this galaxy as $\mathrm{SA}(\mathrm{r}) \mathrm{c}$.

CIG 838. This galaxy is reported as Sbc in NED. This is a clear case of a galaxy that appears multi-armed in the optical images, but that shows only two main arms in the near-infrared. We find evidence of a bar in the optical images that is confirmed by both the $J H K$ image and the geometric profiles. The $B-I$ color map shows the two main arms forming a clumpy pseudo-ring. The total $B-V$ color index is similar to the $\mathrm{Sab} / \mathrm{Sb}$ types. We classify this galaxy as SB(r)bc. Notice the apparently disturbed end of the arm at the west. This object has been flagged as possibly interacting by Sulentic et al. (2006).

CIG 840. The NED classification for this galaxy is $\mathrm{SB}(\mathrm{s}) \mathrm{b}$. There are several field-stars. A pair of two main arms emerge from an inner ring. These arms become fragmented in the outer regions. The $R$ band filter-enhanced image gives evidence of a bar, which is confirmed in the $J H K$ image and the geometric profiles. The total $B-V$ color index is similar to the $\mathrm{Sa} / \mathrm{Sab}$ types. We classify this galaxy as $\mathrm{SB}(\mathrm{r}) \mathrm{bc}$.

CIG 858. NED reports this galaxy as SBb. Two prominent arms are shown in the optical images that appear blue in the $B-$ $I$ color map. We find evidence of a bar in both the optical and the $J H K$ images. This is reinforced by the geometric profiles. A dust lane dividing the southern arm into two components is evident in the optical images. The total $B-V$ color index is consistent with $\mathrm{SOa} / \mathrm{Sa}$ and $\mathrm{Sab} / \mathrm{Sb}$ types. We classify this galaxy as $\mathrm{SBb}$.

CIG 861. The NED classification for this galaxy is SBbc. The optical images show two prominent arms and an elongated central region that is not aligned with the bar. The composed $J H K$ image shows only the central region, and a bar that is confirmed by the geometric profiles. The total $B-V$ color index is representative of the $\mathrm{Sbc/Sc}$ types. Our classification for this galaxy is SBbc.

CIG 862. NED reports this galaxy as SBbc. A pair of arms emerging from the south side of a bar are appreciated in the optical images. In contrast, the $J H K$ image shows the arms emerging from opposite sides of the bar. These arms become fragmented and clumpy in the outer regions. The geometric profiles confirm the bar. The total $B-V$ color index is similar to the $\mathrm{Sab} / \mathrm{Sb}$ types. $\mathrm{SBbc}$ is our classification for this galaxy.

CIG 947. The NED classification for this galaxy is (R)SA(r)ab; Sy LINER. In the optical images we appreciate a tight spiral structure and a ring in the inner regions. The $B-I$ color map shows uniform colors inside, and redder colors in the arms. The total $B-V$ color index is similar to the $\mathrm{S} 0 \mathrm{a} / \mathrm{Sa}$ types. The composed $J H K$ image shows a smooth central region. We classify this galaxy as $\mathrm{SA}(\mathrm{r}) \mathrm{ab}$.

\section{Optical morphology: results}

Table 5 is a summary of the morphological results found in this work. Column (1) gives the CIG catalog number, Col. (2) gives the Hubble type as reported in NED, Col. (3) gives the Hubble type as estimated in this work, Col. (4) indicates the presence of bars/rings, and Col. (5) reports the bar ellipticity (corrected for inclination). Finally, Col. (6) gives the apparent inclination, taken from LEDA.

NED contains morphological information on subtypes for all these isolated galaxies. From our image analysis, we find that $37.5 \%$ of the galaxies are earlier than Sbc and $62.5 \%$ are of Sbc type or later. The catalog information concerning bars (confirmed and presumed) comprised 19 galaxies before this work, and we were able to add this information to 7 other galaxies. This indicates that up to $65 \%$ of the isolated galaxies in this subsample show evidence of barred structure: for $40 \%$ the evidence is clear (SB galaxies), and for $25 \%$ the bars are weak or suspected (SAB galaxies). The bar fraction is $68 \%$ for latetypes and $60 \%$ for early-types. We have measured the $R$-band and $J H K$ isophotal ellipticities associated with a bar and estimated the maximum ellipticity, $\epsilon_{\max }$. This quantity (corrected by inclination) is related to a measure of bar strength, such as gravitational bar torque (Laurikainen et al. 2002). Column 5 in Table 5 gives the values of $\epsilon_{\max }$ for our sample. Among barred galaxies, the average value of $\epsilon_{\max }$ is $0.35 \pm 0.13$. Two earlytype galaxies and four late-type galaxies have $\epsilon_{\max } \geq 0.4$, which is commonly considered as evidence of a strong bar. Similarly, the catalog information for rings in our sample previously comprised 16 galaxies; in this work, this information has been added to 7 other galaxies, now accounting for $57.5 \%$ of the sample. Notice in Table 5, that galaxies with inclination higher than $80^{\circ}$ may not have a reliable classification.

\subsection{Morphology, bars, and rings in isolated galaxies}

The reclassification presented here (see Table 5) preserves the optically observed morphology but takes into account the NIR bar morphology. In general, the results concerning differences in morphology when passing from the optical to the NIR bands (Eskridge et al. 2000) agree with those in our subsample of isolated galaxies. While the reported fraction of barred galaxies in the optical is almost a factor of 2 smaller than that in the NIR (Eskridge et al. 2000), in our case the fraction is actually small $(\sim 20 \%)$.

The fraction of galaxies in our sample with clear evidence of optical/IR bars (SB galaxies) is $40 \%$, while $25 \%$ more show some evidence of weak bars (SAB galaxies). These fractions are in agreement with estimates from other samples of galaxies (Eskridge et al. 2000; Hernández-Toledo et al. 2007; Verley et al. 2007). We also report the presence of inner $(r)$ and outer $(R)$ rings when possible, but a detailed ring morphology (Buta 1986, 1995) was not attempted. The fraction of galaxies with rings in our sample is $57.5 \%$. Note that the observed fraction of bars and rings in the present paper can hardly be a bias of our observing procedure, since we simply selected objects according to their availability in the sky.

Bars can be characterized as strong when they are long (relative to the scale-length of the disk), have large $m=2,4$ Fourier density amplitudes (typical values for the relative component $m=2$ between $0.4-0.8$, Athanassoula \& Misiriotis 2002), and also when they have high ellipticities since the orbital families of bars strongly depend on the underlying gravitational potential. On the other hand, high-resolution $N$-body simulations of isolated disks embedded in CDM halos show that extended strong bars almost always form, but they slow down as a result of angular momentum transport to the disk and halo (Debattista \& Sellwood 2000; Athanassoula \& Misiriotis 2002; 
Table 5. Final morphological classification.

\begin{tabular}{|c|c|c|c|c|c|}
\hline CIG & Type (NED) & Type (This work) & Bars/Rings & Bar ellipticity $\epsilon_{\max }$ & Inclination \\
\hline CIG 72 & SA(rs)b Sy1.9 & $\mathrm{SB}(\mathrm{r}) \mathrm{b}$ & $\mathrm{B} / \mathrm{R}$ & 0.286 & 54.77 \\
\hline CIG 89 & E6? & S0 & & & 43.92 \\
\hline CIG 103 & $\mathrm{SAB}(\mathrm{rs}) \mathrm{c}$ & $\mathrm{SAB}(\mathrm{r}) \mathrm{cd}$ & $\mathrm{B} / \mathrm{R}$ & 0.464 & 57.55 \\
\hline CIG 109 & $\mathrm{SA}(\mathrm{rs}) \mathrm{b}$ & (R)SA(s)ab & $\mathrm{R}$ & & 68.21 \\
\hline CIG 135 & SBa & SB(r)ab & $B / R$ & 0.327 & 51.15 \\
\hline CIG 145 & Sab & $\mathrm{Sa}$ & & & 80.69 \\
\hline CIG 156 & SB(r)ab pec & (R)SBbc & $B / R$ & 0.482 & 42.06 \\
\hline CIG 191 & (R')SBa & (R)SBa & $\mathrm{B} / \mathrm{R}$ & 0.255 & 65.86 \\
\hline CIG 199 & Scd & $\mathrm{SAB}(\mathrm{r}) \mathrm{cd}$ & $\mathrm{B} / \mathrm{R}$ & 0.321 & 72.21 \\
\hline CIG 309 & $\mathrm{SA}(\mathrm{r}) \mathrm{ab}$ & $\mathrm{SA}(\mathrm{r}) \mathrm{a}$ & $\mathrm{R}$ & & 40.60 \\
\hline CIG 314 & $\mathrm{SAB}(\mathrm{rs}) \mathrm{c}$ & SA(r)d & $\mathrm{R}$ & & 67.33 \\
\hline CIG 392 & S0 & $\mathrm{SB}(\mathrm{r}) \mathrm{a}$ & $B / R$ & 0.265 & 39.23 \\
\hline CIG 448 & $\mathrm{SAB}(\mathrm{rs}) \mathrm{c}$ & $\mathrm{SABc}$ & B & - & 72.8 \\
\hline CIG 477 & SA(s)c HII & $\mathrm{Sc}$ & & & 47.55 \\
\hline CIG 491 & SAB(r)ab? & $\mathrm{SAB}(\mathrm{r}) \mathrm{c}$ & $\mathrm{B} / \mathrm{R}$ & - & 52.68 \\
\hline CIG 500 & $\left(\mathrm{R}^{\prime}\right) \mathrm{SAB}(\mathrm{r}) \mathrm{ab}$ & $\mathrm{SB}(\mathrm{r}) \mathrm{b}$ & $\mathrm{B} / \mathrm{R}$ & - & $\ldots$ \\
\hline CIG 507 & Sbc & SABcd & B & - & 78.86 \\
\hline CIG 512 & $\mathrm{SB}(\mathrm{s}) \mathrm{cd}$ & SBcd & B & 0.254 & 29.2 \\
\hline CIG 525 & $\mathrm{SB}(\mathrm{r}) \mathrm{b}$ & $\mathrm{SB}(\mathrm{r}) \mathrm{bc}$ & $\mathrm{B} / \mathrm{R}$ & 0.372 & 42.57 \\
\hline CIG 539 & $\mathrm{Sb}$ & SB(r)ab & $\mathrm{B} / \mathrm{R}$ & 0.243 & 49.37 \\
\hline CIG 547 & $\mathrm{SAB}(\mathrm{s}) \mathrm{d}$ & $\mathrm{Sd}$ & & & 44.34 \\
\hline CIG 575 & $\mathrm{SAB}(\mathrm{rs}) \mathrm{c}$ SBNG & SABc & B & - & 44.86 \\
\hline CIG 616 & $\mathrm{~S} 0 / \mathrm{a}$ & SABab & B & - & 70.95 \\
\hline CIG 624 & SAB(s)d HII & SABdm & B & 0.203 & 53.20 \\
\hline CIG 630 & $\mathrm{Sb}$ & $\mathrm{SA}(\mathrm{rs}) \mathrm{bc}$ & $\mathrm{R}$ & & 58.25 \\
\hline CIG 631 & (R)SA(rs)b & $\mathrm{SA}(\mathrm{r}) \mathrm{bc}$ & $\mathrm{R}$ & & 50.8 \\
\hline CIG 712 & (R)SB(r)ab: LINER: & $\mathrm{SB}(\mathrm{r}) \mathrm{b}$ & $\mathrm{B} / \mathrm{R}$ & 0.467 & 46.77 \\
\hline CIG 716 & $\mathrm{SA}(\mathrm{rs}) \mathrm{bc}$ & $\mathrm{SA}(\mathrm{r}) \mathrm{b}$ & $\mathrm{R}$ & & 37.48 \\
\hline CIG 744 & $\mathrm{Sc}$ & $\mathrm{Sd}$ & & & 32.9 \\
\hline CIG 766 & SA(s)c HII & SABcd & B & - & 70.99 \\
\hline CIG 772 & Scd & $\mathrm{SA}(\mathrm{s}) \mathrm{cd}$ & & & 64.66 \\
\hline CIG 800 & $\mathrm{SBb}$ & $\mathrm{SB}(\mathrm{s}) \mathrm{bc}$ & B & 0.513 & 66.35 \\
\hline CIG 805 & $\mathrm{Sb}$ & $\mathrm{SAB}(\mathrm{rs}) \mathrm{bc}$ & $\mathrm{B} / \mathrm{R}$ & 0.285 & 56.86 \\
\hline CIG 812 & Sbc & $\mathrm{SA}(\mathrm{r}) \mathrm{c}$ & $\mathrm{R}$ & & 41.09 \\
\hline CIG 838 & Sbc & $\mathrm{SB}(\mathrm{r}) \mathrm{bc}$ & $\mathrm{B} / \mathrm{R}$ & 0.289 & 51.48 \\
\hline CIG 840 & $\mathrm{SB}(\mathrm{s}) \mathrm{b}$ & $\mathrm{SB}(\mathrm{r}) \mathrm{bc}$ & $\mathrm{B} / \mathrm{R}$ & 0.348 & 46.46 \\
\hline CIG 858 & $\mathrm{SBb}$ & $\mathrm{SBb}$ & B & 0.601 & 59.23 \\
\hline CIG 861 & SBbc & SBbc & B & 0.541 & 64.43 \\
\hline CIG 862 & SBbc & SBbc & B & 0.156 & 24.96 \\
\hline CIG 947 & (R)SA(r)ab; Sy LINER & $\mathrm{SA}(\mathrm{r}) \mathrm{ab}$ & $\mathrm{R}$ & & 36.2 \\
\hline
\end{tabular}

Valenzuela \& Klypin 2003); eventually, the bars may dissolve, forming a pseudo bulge (e.g., Avila-Reese et al. 2005; Berentzen et al. 2006).

For our sample, we have estimated the bar de-projected maximum ellipticity, $\epsilon_{\max }$. We do not find differences in $\epsilon_{\max }$ as a function of morphological type.

\subsection{Distorted morphology and bright/dark galaxies}

As discussed in Karachentsev et al. (2006) and according to recent cosmological models, apart from dark halos with normal (luminous) galaxies in their centers, completely dark clumps (sub-halos) should also exist (van den Bosch et al. 2003; Tully 2005; Yang et al. 2005). Apparently, the completely dark galaxies (subhalos) may be detected via gravitational effects (Trentham et al. 2001), thus providing the possibility that the phenomena of interaction will occur even in the case of an isolated galaxy. Hence, a relative number of peculiar shapes among our isolated galaxies may be expected.

We inspected our images to identify visible distortions, asymmetries and structures resembling a tidal origin and/or possible double nuclei or recent merger candidates. Thin open and relatively featureless structures not associated to any underlying disk, and distorted spiral arms, were also interpreted as interaction-induced features. We have classified our sample according to moderate and strong distortions. In Fig. 4 we show 5 cases, among our 40 galaxies, where we have found moderated distortions: CIG 109, CIG 135, CIG 539, CIG 631, and CIG 744 (see Figs. B.3, B.4, B.19, B.25 and B.28 in the electronic version), in the form of slightly distorted arms, low surface brightness and asymmetric bright halos, and a peculiar case of a galaxy with a central clumpy region surrounded by a smooth symmetric envelope.

In contrast, Fig. 5 shows 4 cases of galaxies where the evidence of morphological distortions was stronger: CIG 72, CIG 716, CIG 812, and CIG 838 (see Figs. 3, B.27, B.33, and B.34 in the electronic version), in the form of clear asymmetries and thin filamentary arms resembling tidal features, similar to those features reported in Karachenstsev et al. (2007).

Table 6 reports some structural, photometric and gas content properties for our 40 CIG galaxies that are useful to understand something about the physical nature of these 9 morphologicallydistorted isolated galaxies. Column (1) gives the original CIG number, Col. (2) gives the absolute $I$ band magnitude (from 

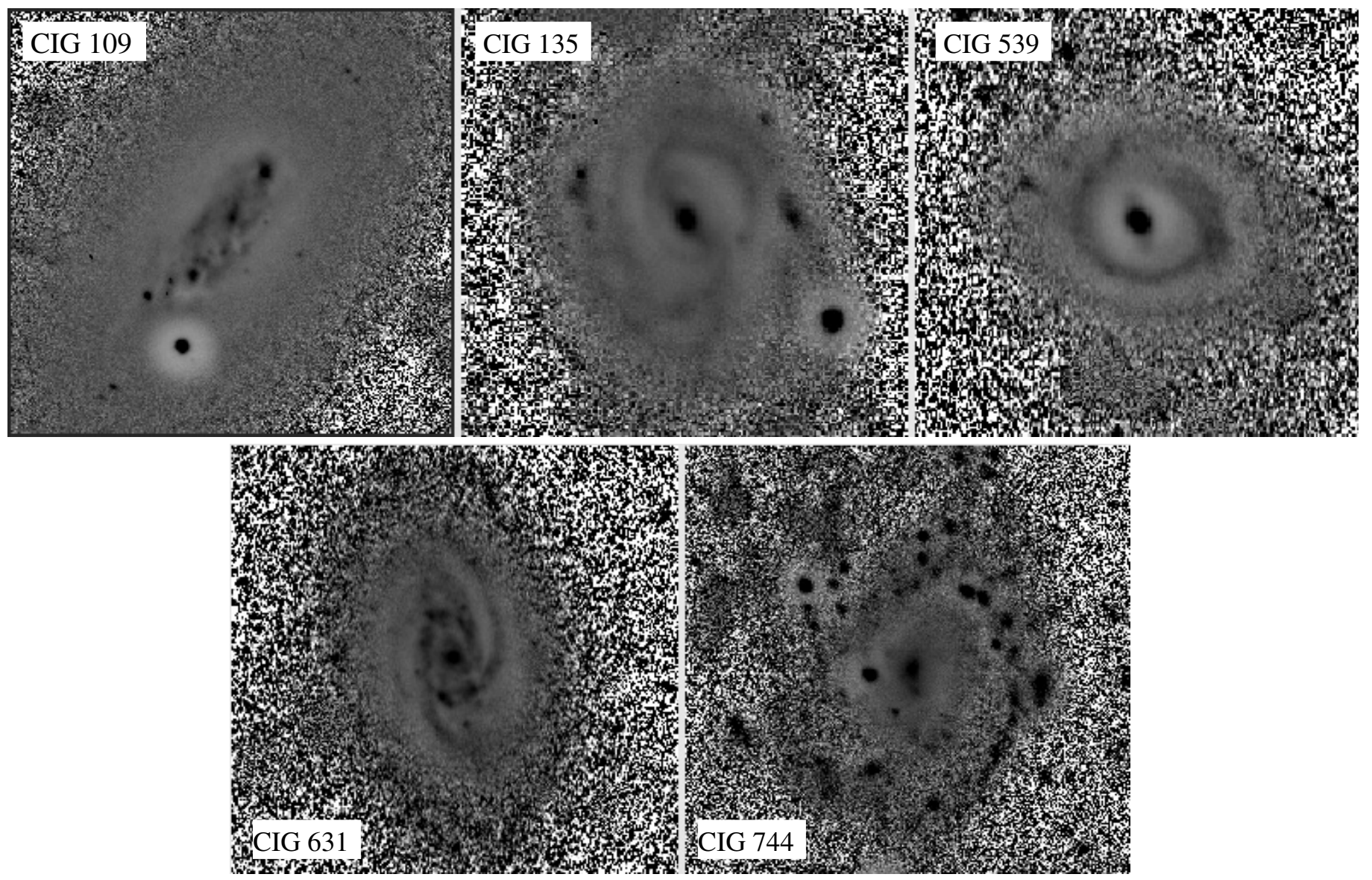

Fig. 4. Galaxies with moderated morphological distortions, CIG 109, CIG 135, CIG 539, CIG 631 and CIG 744. $R$-band filter-enhanced images.

our photometry data), Col. (3) gives the $M_{\mathrm{HI}} / L_{I}$ ratio estimated from our photometry and from data available in the HyperLeda database, Col. (4) gives the central disk surface brightness in the $I$ band (from our surface photometry) and Col. (5) is the category in which the 9 distorted galaxies fall (in accordance to Karachenstsev et al. 2007): (A) galaxies with ripples, plumes, loops or tidal tails caused apparently by a recent merging/close encounter; (B) galaxies of asymmetric shape and star formation, (C) galaxies with peculiar or distorted arms.

In most of the cases, the morphological distortions of the galaxies in Table 6 are moderate and could be evidencing some level of dynamical disturbance.

Although the CIG selection criteria warranties that these galaxies have not suffered perturbations of similar size galaxies over some gigayears, it is now known that the presence of small neighbors could cause a notable effect on the observed morphology. Verley et al. (2007) quantified the isolation degree for all the CIG sample providing 2 parameters (the local number density of neighbor galaxies of comparable or smaller size and the corresponding tidal strength) to estimate the influence of neighbor galaxies on the evolution, and in particular on the observed morphology. Furthermore, by using the complementarity of these parameters, the CIG sample was refined from 950 to 750 galaxies proposed as more dominated by their intrinsic properties.

Our results yield 9 distorted galaxy candidates, 5 of them are also in the list given by Sulentic et al. (2006) of possible interacting candidates in the CIG sample. These 9 distorted candidates were first reviewed for the local number density and tidal strength parameters, finding that 4 of them violate the refinement criteria proposed by Verley et al. (2007) (CIG 72, 539, 631 and 812 ).

Figure 6 shows a plot of the local number density $\left(\eta_{\mathrm{k}}\right)$ versus the tidal strength $(Q)$ for the refined sample of 791 more likely isolated galaxies from the CIG galaxies. The data has been taken from the published Table 4 in Verley et al. (2007). The solid line and the adjacent dashed lines represent a least square fit to the data along with the corresponding 2 sigma region. Solid triangles represent the position of the morphologically distorted galaxies (CIG 135, CIG 744 and CIG 838) found in our study that do not violate the refinement criteria proposed by Verley et al. (2007) (that is, $Q>-2$ and $\eta_{\mathrm{k}}>2.4$ ). The solid pentagon symbol represents the position of CIG 716. Notice that since there is no estimate of $\eta_{\mathrm{k}}$ for this galaxy, we adopted the mean value for the given $Q$. Figure 6 shows that all our candidates fall within the 2 sigma dispersion limits despite of being morphologically distorted.

From Table 6, we see that CIG 744 has $M_{\mathrm{HI}} / L_{I}>1$. From our surface photometry, the disk parameters $\mu_{0}$ (central surface brightness) and $h$ (scale length) were estimated. We also have explored their trends with other global properties like the gas content or the absolute luminosity. Although it is not the scope of this paper to present a detailed analysis of these correlations, here we will focus on a correlation found between the central disk surface brightness and the content of gas as devised by the $M_{\mathrm{HI}} / L_{B}$ and $M_{\mathrm{HI}} / L_{I}$ ratios.

Figure 7 shows the mass-to-light ratio as a function of disk central surface brightness in the $B$ and $I$ bands. A strong correlation between the gas content of the spirals in our study and their surface brightness is appreciated. To reinforce the correlation, 


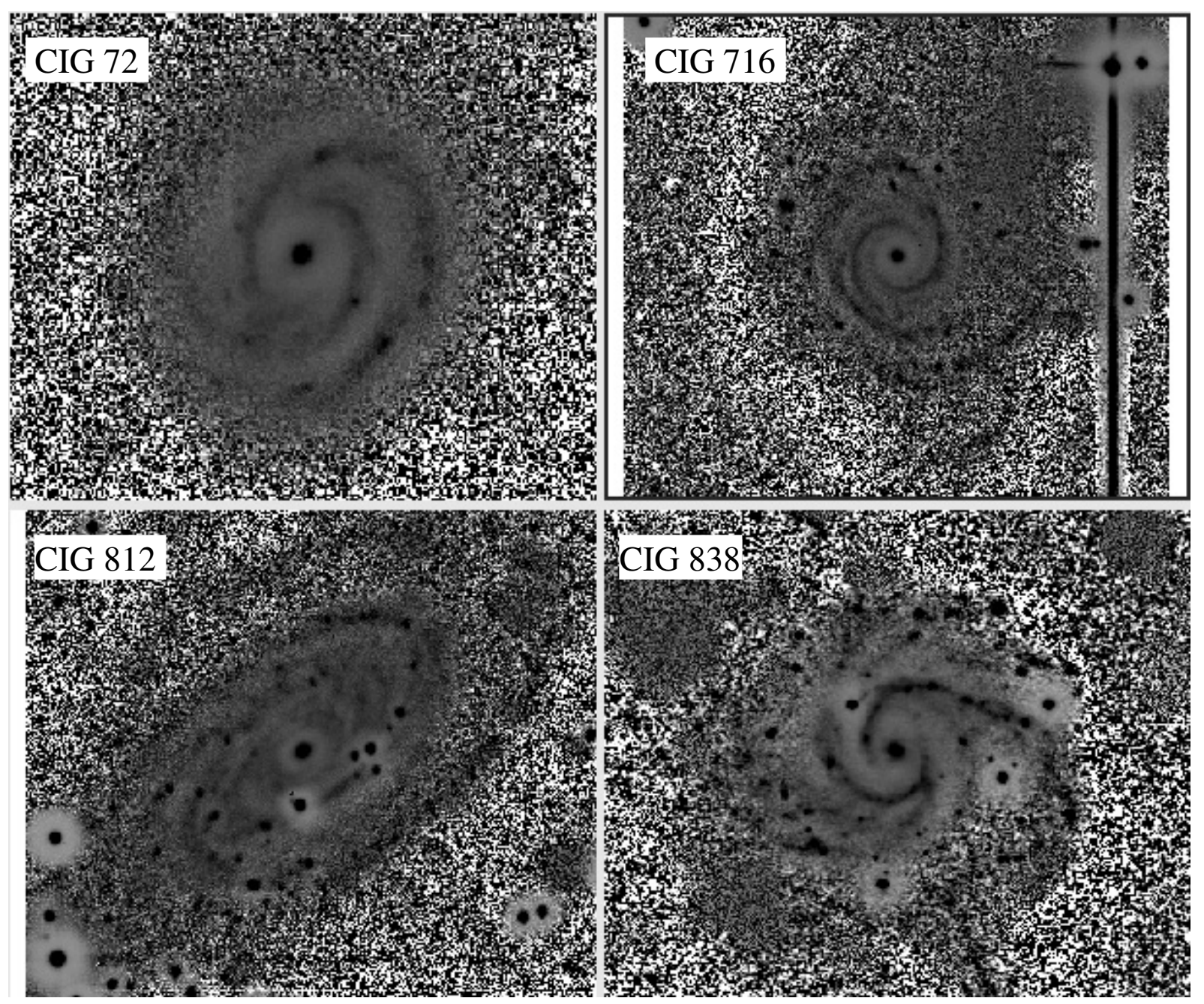

Fig. 5. Galaxies with strong morphological distortions, CIG 72, CIG 716, CIG 812 and CIG 838. $R$-band filter-enhanced images.

we have included the data in McGaugh \& de Blok (1997) for a sample of field spiral galaxies. Figure 7 also shows the loci of our 9 morphologically distorted galaxies. Surprisingly CIG 744 is an example of a galaxy in our sample with a very low surface brightness disk and with intrinsically high gas-content.

As discussed in McGaugh \& de Blok (1997), there could be some bias behind that correlation but from Tables 5 and 6, it is clear that the galaxies in our study show a wide range in luminosity, span a wide range of morphologies from Sa to $\mathrm{Sm} / \mathrm{Im}$ and also (Fig. 5) a wide range in surface brightness, allowing us to have a less-biassed interpretation. This correlation reflects real differences in the gas content of all these galaxies and emphasizes the role of the surface brightness of a disk as a fundamental parameter and good indicator of the evolutionary status of a galaxy (Zavala et al. 2003; and Avila-Reese et al. 2008). According to McGaugh \& de Blok (1997) lower surface brightness and lower luminosity spirals have consumed less of their gas and are less evolved than brighter systems and perhaps form late. On line to this, CIG 744 is a gas-rich but possibly a lessevolved system than the other galaxies in our sample. Although it is still possible to invoke the existence of dark objects in the neighborhood of CIG 744 as an explanation for its observed distorted morphology, it is first necessary to carefully review the intrinsic physical properties of this galaxy and furthermore, explore other possible causes, to mention; minor mergers in its neighborhood. We notice that from the SDSS images, several dwarf galaxies can still be appreciated, and that, in addition, this object has been flagged as possibly interacting by Sulentic et al. (2006).

\section{Physical morphology}

Physical morphology has emerged as a useful framework for classifying galaxies on the basis of their underlying physical properties (Morgan \& Osterbrock 1969; Abraham et al. 1996; Conselice 1997; Bershady et al. 2000). More recently, Conselice (2003, hereafter C03) has shown that the major ongoing and past formation modes of galaxies can be distinguished by using three model-independent structural (photometric) parameters, which allow to have a robust classification system. These parameters are the concentration of stellar light $(C)$, its asymmetric distribution $(A)$, and a measure of its clumpiness $(S)$.

We present the $C A S$ parameters measured at various passband for our observed sample of isolated galaxies. The measurement of the CAS parameters for the isolated spiral galaxies was carried out in several steps:

(1) close field stars were removed from each image; (2) sky background was removed from the images; (3) the center of each galaxy was considered as the barycenter of the light distribution and the starting point for measurements; (4) the CAS parameters for all the spiral isolated galaxies were estimated directly, i.e. isolated galaxies were not influenced by light contamination 
Table 6. Luminosity, gas content, central disk surface brightness and category of morphology distortion.

\begin{tabular}{ccccc}
\hline \hline CIG & $M_{\text {I }}$ & $M_{\text {HI }} / L_{I}$ & $\mu_{0}^{\text {I }}$ & Category \\
\hline CIG 72 & -22.261 & 0.083 & 19.25 & A \\
CIG 89 & -22.529 & - & - & - \\
CIG 103 & -21.511 & 0.125 & 19.5 & - \\
CIG 109 & -19.547 & 0.167 & 18.25 & B \\
CIG 135 & -22.451 & 0.175 & 19.0 & A \\
CIG 145 & -22.088 & 0.024 & 18.75 & - \\
CIG 156 & -21.375 & 0.028 & 17.5 & - \\
CIG 191 & -23.315 & 0.044 & 18.0 & - \\
CIG 199 & -21.486 & 0.283 & 19.0 & - \\
CIG 309 & -22.626 & 0.006 & - & - \\
CIG 314 & - & - & - & - \\
CIG 392 & -22.495 & - & 19.0 & - \\
CIG 448 & -21.033 & 0.080 & 17.5 & - \\
CIG 477 & -21.134 & 0.073 & 17.75 & - \\
CIG 491 & -22.201 & 0.142 & 18.75 & - \\
CIG 500 & -23.584 & 0.045 & 19.75 & - \\
CIG 507 & -21.379 & 0.256 & 20.0 & - \\
CIG 512 & -20.390 & 0.333 & 21.0 & - \\
CIG 525 & -23.158 & 0.123 & 19.75 & - \\
CIG 539 & -22.633 & 0.317 & 18.5 & A \\
CIG 547 & -18.978 & 0.124 & 20.0 & - \\
CIG 575 & -21.589 & 0.134 & 18.5 & - \\
CIG 616 & -23.461 & - & 19.5 & - \\
CIG 624 & -19.578 & 0.255 & - & - \\
CIG 630 & -21.709 & 0.163 & 18.75 & - \\
CIG 631 & -21.463 & 0.143 & 18.0 & B \\
CIG 712 & -21.280 & 0.155 & 18.75 & - \\
CIG 716 & -23.330 & 0.291 & 20.5 & A \\
CIG 744 & -19.925 & 1.052 & 22.75 & C \\
CIG 766 & -20.537 & 0.146 & 18.0 & - \\
CIG 772 & -22.707 & 0.002 & 19.0 & - \\
CIG 800 & -23.051 & 0.068 & 18.5 & - \\
CIG 805 & -22.103 & 0.275 & 19.25 & - \\
CIG 812 & -22.507 & 0.304 & 19.0 & A, B \\
CIG 838 & -22.952 & 0.207 & 19.5 & A \\
CIG 840 & -22.595 & 0.093 & 19.25 & - \\
CIG 858 & -22.723 & 0.121 & 19.0 & - \\
CIG 861 & -21.817 & - & 19.25 & - \\
\hline & -22.219 & 0.081 & 19.0 & - \\
\hline & - & - & - & - \\
\hline CIG 947 & & &
\end{tabular}

from any other galaxy of similar size in the neighborhood (isolation criteria); and (5) galaxies with high inclinations or axis ratios could introduce systematic biased trends in the values of the CAS parameters (C03). Isolated galaxies whose apparent axial ratios yield "inclinations" higher than $80^{\circ}$ are represented by open circles in the corresponding plots.

\subsection{CAS results}

In the present analysis we use the $R$ band $C A S$ parameters and their errors (Table 7). The $C A S$ values in the other bands can be provided upon request. Column (1) in Table 7 gives the CIG catalog number, and Cols. (2)-(4) report the $C A S$ values with their respective errors, in the $R$-band.

By sorting the sample into early- and late-type spirals ( $\mathrm{SaSb}$ and $\mathrm{SbcSm}$, respectively), the corresponding average

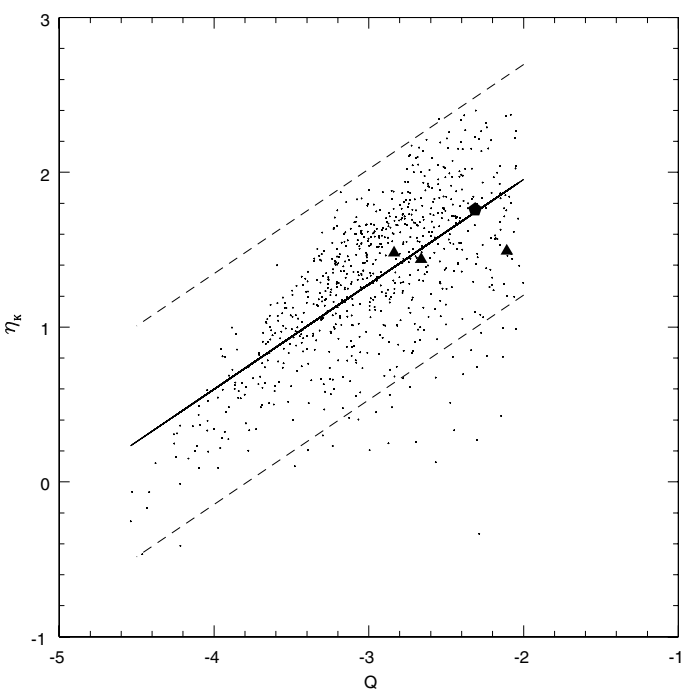

Fig. 6. Local number density $\eta_{\mathrm{k}}$ versus tidal strength $Q$. Solid triangle symbols represent the position of the morphologically distorted galaxies that do not violate the $Q>-2$ rule; the solid pentagon symbol represents CIG 716. Since there is no estimate of $\eta_{\mathrm{k}}$ for this galaxy, we adopted the mean value for the given $Q$.
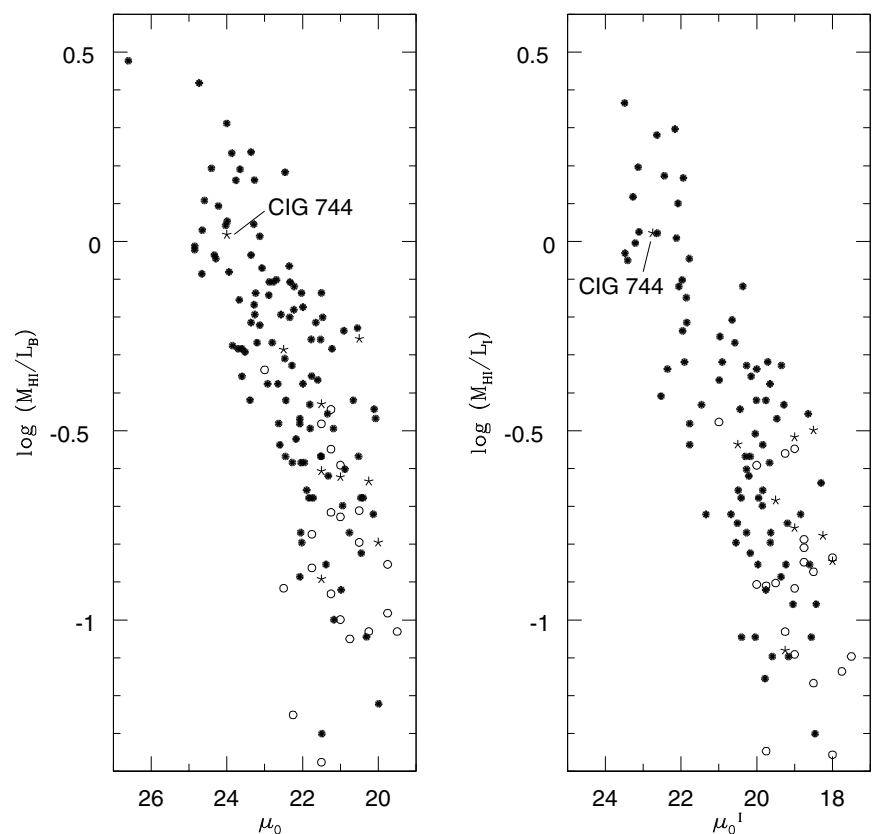

Fig. 7. HI mass-to-light ratio as a function of disk central surface brightness: $B$ band (left), I band (right). Solid circles are for data taken from McGaugh \& de Blok (1997) for a sample of field spiral galaxies; open circles represent the CIG galaxies from this study and star symbols represent our 9 morphologically distorted galaxies.

and standard deviation values of the $C A S$ parameters are: $\langle C(R)\rangle(\mathrm{SaSb})=3.74 \pm 0.40,\langle A(R)\rangle(\mathrm{SaSb})=0.09 \pm 0.06$, $\langle S(R)\rangle(\mathrm{SaSb})=0.23 \pm 0.21$, and $\langle C(R)\rangle(\mathrm{SbcSm})=3.19 \pm 0.36$, $\langle A(R)\rangle(\mathrm{SbcSm})=0.16 \pm 0.07,\langle S(R)\rangle(\mathrm{SbcSm})=0.38 \pm 0.23$. Our mean values are consistent with those reported in $\mathrm{C} 03$ for the Frei et al. (1996) sample of non-interacting galaxies and also with those reported in Hernández-Toledo et al. (2007). Note that irregulars were included in SbcSm class, while in C03 these galaxies are separated. 
Table 7. $R$-Band $C A S$ parameters for isolated spiral galaxies.

\begin{tabular}{|c|c|c|c|}
\hline CIG & $C(R)$ & $A(R)$ & $S(R)$ \\
\hline CIG 72 & $3.209 \pm 0.097$ & $0.091 \pm 0.019$ & $-0.05 \pm 0.002$ \\
\hline CIG 89 & $4.28 \pm 0.061$ & $0.006 \pm 0.008$ & $0.09 \pm 0.01$ \\
\hline CIG 103 & $3.052 \pm 0.044$ & $0.245 \pm 0.016$ & $0.60 \pm 0.011$ \\
\hline CIG 109 & $3.491 \pm 0.075$ & $0.206 \pm 0.007$ & $0.54 \pm 0.006$ \\
\hline CIG 135 & $3.443 \pm 0.128$ & $0.039 \pm 0.008$ & $0.07 \pm 0.002$ \\
\hline CIG 145 & $3.409 \pm 0.139$ & $0.275 \pm 0.011$ & $0.26 \pm 0.004$ \\
\hline CIG 156 & $3.403 \pm 0.086$ & $0.252 \pm 0.003$ & $0.62 \pm 0.008$ \\
\hline CIG 191 & $4.131 \pm 0.169$ & $0.075 \pm 0.009$ & $0.17 \pm 0.003$ \\
\hline CIG 199 & $3.292 \pm 0.104$ & $0.107 \pm 0.019$ & $0.37 \pm 0.01$ \\
\hline CIG 309 & $3.892 \pm 0.06$ & $0.011 \pm 0.008$ & $0.05 \pm 0.004$ \\
\hline CIG 314 & $3.306 \pm 0.058$ & $0.17 \pm 0.014$ & $0.16 \pm 0.008$ \\
\hline CIG 392 & $3.824 \pm 0.233$ & $0.096 \pm 0.007$ & $0.08 \pm 0.002$ \\
\hline CIG 448 & $3.357 \pm 0.094$ & $0.208 \pm 0.006$ & $0.33 \pm 0.004$ \\
\hline CIG 477 & $3.672 \pm 0.132$ & $0.21 \pm 0.004$ & $0.56 \pm 0.005$ \\
\hline CIG 491 & $3.05 \pm 0.152$ & $0.092 \pm 0.013$ & $0.02 \pm 0.002$ \\
\hline CIG 500 & $3.519 \pm 0.106$ & $0.021 \pm 0.03$ & $-0.07 \pm 0.01$ \\
\hline CIG 507 & $3.483 \pm 0.115$ & $0.108 \pm 0.036$ & $0.38 \pm 0.02$ \\
\hline CIG 512 & $2.602 \pm 0.035$ & $0.026 \pm 0.066$ & $-0.06 \pm 0.03$ \\
\hline CIG 525 & $3.828 \pm 0.109$ & $0.174 \pm 0.009$ & $0.46 \pm 0.008$ \\
\hline CIG 539 & $4.205 \pm 0.196$ & $0.056 \pm 0.006$ & $0.06 \pm 0.002$ \\
\hline CIG 547 & $2.827 \pm 0.049$ & $0.058 \pm 0.038$ & $0.37 \pm 0.01$ \\
\hline CIG 575 & $3.167 \pm 0.089$ & $0.058 \pm 0.013$ & $0.06 \pm 0.003$ \\
\hline CIG 616 & $4.433 \pm 0.19$ & $0.11 \pm 0.004$ & $0.08 \pm 0.002$ \\
\hline CIG 624 & $3.328 \pm 0.041$ & $0.308 \pm 0.012$ & $0.71 \pm 0.008$ \\
\hline CIG 630 & $3.094 \pm 0.104$ & $0.154 \pm 0.01$ & $0.46 \pm 0.01$ \\
\hline CIG 631 & $2.783 \pm 0.114$ & $0.091 \pm 0.007$ & $0.25 \pm 0.005$ \\
\hline CIG 712 & $3.635 \pm 0.046$ & $0.182 \pm 0.014$ & $0.44 \pm 0.014$ \\
\hline CIG 716 & $3.849 \pm 0.05$ & $0.106 \pm 0.052$ & $0.07 \pm 0.025$ \\
\hline CIG 744 & $3.898 \pm 0.083$ & $0.203 \pm 0.062$ & $0.18 \pm 0.011$ \\
\hline CIG 766 & $2.96 \pm 0.076$ & $0.243 \pm 0.005$ & $0.60 \pm 0.006$ \\
\hline CIG 772 & $3.129 \pm 0.089$ & $0.065 \pm 0.018$ & $0.14 \pm 0.005$ \\
\hline CIG 800 & $2.675 \pm 0.091$ & $0.238 \pm 0.008$ & $0.59 \pm 0.009$ \\
\hline CIG 805 & $3.066 \pm 0.098$ & $0.169 \pm 0.022$ & $0.17 \pm 0.006$ \\
\hline CIG 812 & $3.543 \pm 0.063$ & $0.164 \pm 0.018$ & $0.60 \pm 0.009$ \\
\hline CIG 838 & $3.26 \pm 0.067$ & $0.291 \pm 0.023$ & $0.68 \pm 0.017$ \\
\hline CIG 840 & $2.52 \pm 0.065$ & $0.235 \pm 0.005$ & $0.59 \pm 0.014$ \\
\hline CIG 858 & $3.388 \pm 0.125$ & $0.053 \pm 0.013$ & $0.43 \pm 0.006$ \\
\hline CIG 861 & $3.403 \pm 0.311$ & $-0.053 \pm 0.277$ & $0.11 \pm 0.001$ \\
\hline CIG 862 & $2.97 \pm 0.101$ & $0.323 \pm 0.01$ & $0.57 \pm 0.008$ \\
\hline CIG 947 & $3.748 \pm 0.066$ & $0.083 \pm 0.011$ & $0.05 \pm 0.003$ \\
\hline
\end{tabular}

Figure 8 shows the cumulative distribution function of the $C A S$ parameters at the $B, R, J$ and $K$ bands. The concentration $C$ becomes higher from bluer to redder bands. In the case of the asymmetry $A$ and clumpiness $S$ parameters, their values decrease from bluer to redder bands.

In Fig. 9 we plot the average and standard deviation values of the $C A S$ parameters versus wavelength for our sample, sorted into early- and late-type spirals (SaSb (left panel) and SbcSm (right panel), respectively). The CAS parameters of later types show, on average, slightly more dependence on wavelength than the early types. Among the $C A S$ parameters, clumpiness is the most sensitive to wavelength, although a high scatter is observed in this parameter.

Despite the small number of galaxies in our current sample, we introduce an indicative discussion of the measured $C A S$ parameters in different color bands and their trends with other galaxy properties.

Figure 10 shows the loci of the isolated $\mathrm{SaSb}$ and $\mathrm{SbcSm}$ galaxies in the projected planes of the $R$-band $C A S$ space.

The large boxes indicate the amplitude of variation of the $C A S$ parameters (lower and upper limits) from the $B$ up to the $K$ bands. For comparison, the $R$-band averages
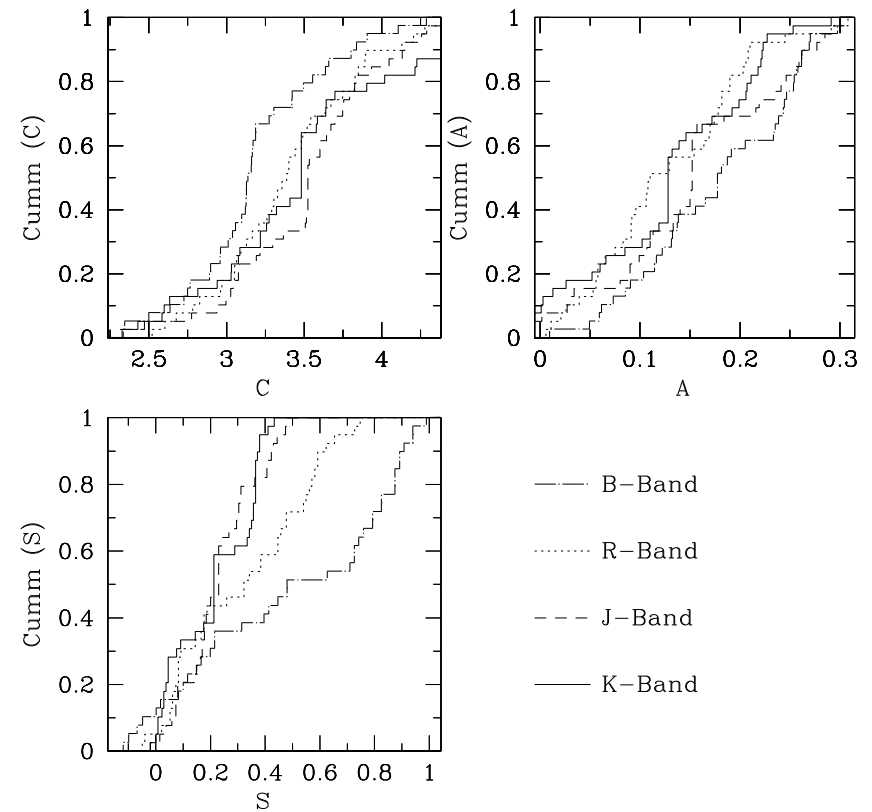

-.. B-Band

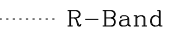

$---J-B a n d$

K-Band

Fig. 8. Cumulative distribution function of $C A S$ parameters in the bands $B, R, J$ and $K$ bands.

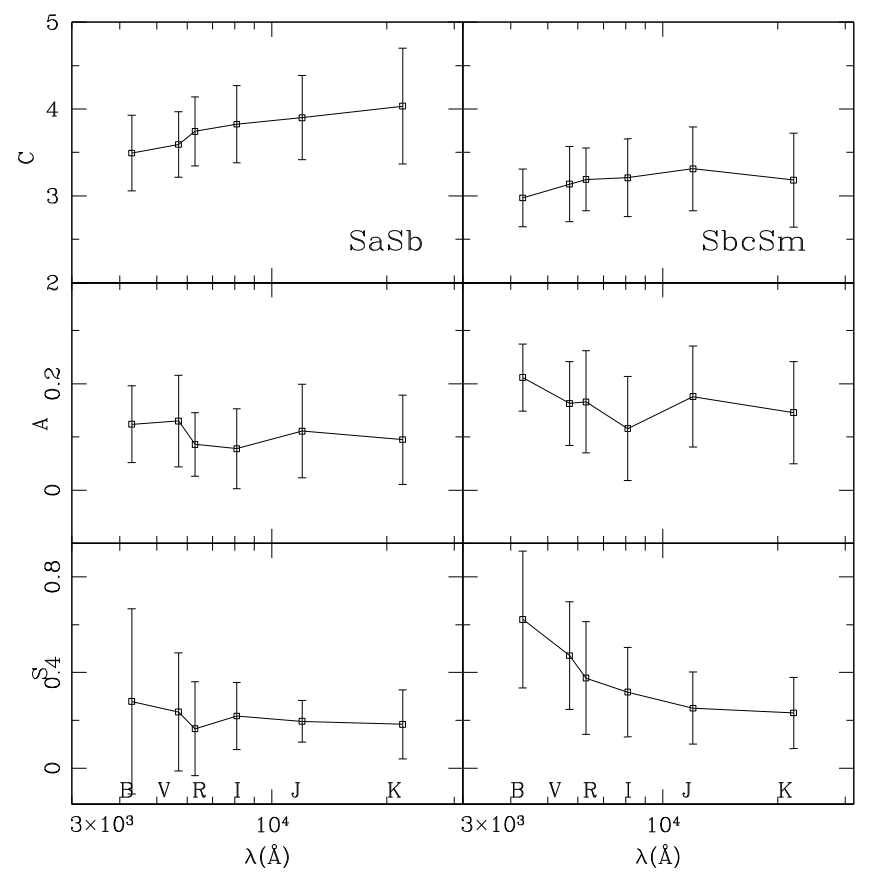

Fig. 9. Average and standard deviation values of the CAS parameters as a function of the central wavelength $\lambda(\AA)$. Left and right columns are for the subsamples of early $(\mathrm{SaSbc})$ and late $(\mathrm{ScSm})$ isolated galaxies.

and standard deviations of galaxies in interacting $\mathrm{S}+\mathrm{S}$ pairs (Hernández-Toledo et al. 2005), and starburst and ultra luminous infrared galaxies (ULIRGs) (C03) are also plotted.

As appreciated in Fig. 10, the representative values of the $C A S$ parameters for our isolated galaxies are significantly lower than the correspondent values observed in interacting/starburst galaxies (Hernández-Toledo et al. 2005). 


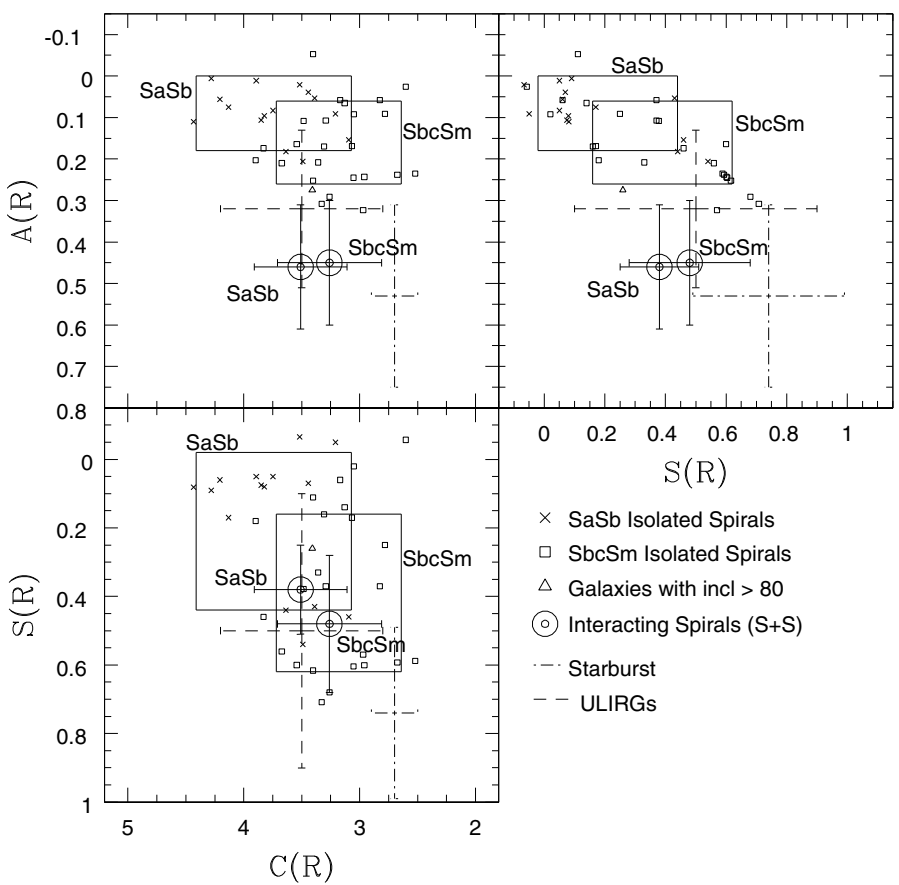

Fig. 10. Loci of the $R$-band $C A S$ values and their $1 \sigma$ dispersion for our isolated $\mathrm{SaSb}$ (crosses) and $\mathrm{SbcSm}$ (open squares) galaxies in the $C A S$ planes. The large boxes illustrate the amplitude of variation of the $C A S$ values from all the bands $(B, V, R, I$ to $J$ and $K$, as estimated in this work). The corresponding $R$-band values for the interacting $\mathrm{SaSb}$ and $\mathrm{SbcSm}$ galaxies are shown with circles and solid error bars (Hernández-Toledo et al. 2005). Dot-dash-dot and long-dash error bars are for ULIR and starburst galaxies, respectively (C03).

\subsubsection{Concentration}

The quantitative measure of $C$ in our isolated spirals span the range $2.8 \leq C(R) \leq 4.2$. The average and standard deviation values are $\langle C(R)\rangle=3.4 \pm 0.47$. We have also found that in general $C$ systematically increases with the passband (Fig. 9).

According to our current understanding of galaxy formation, disks generally form from the inside out within growing CDM halos (Avila-Reese 2006). Their concentrations (or surface brightnesses) depend mainly on the spin parameter of the halo. CDM halos span a wide log-normal distribution of the spin parameter; hence, one also expects a wide range of concentrations for the disks. Most likely, the observed distribution of concentrations for isolated disk galaxies is not as wide as we would expect from theory. It should also be taken into account that internal secular processes after disk formation rearrange the mass (light) distribution, and that the presence of a big bulge in earlytype spirals tends to increase their $C$-parameter with respect to galaxies with smaller bulges. In the case of ellipticals (and probably the bulges of early-type galaxies), theory suggests that they are more concentrated due to the violent and dissipative processes that are at the basis of their formation: major mergers of gaseous disks.

\subsubsection{Asymmetry}

Some studies have shown that important deviations from axisymmetry exist in the optical and other wavelengths (Rix \& Zaritsky 1995; Richter \& Sancisi 1994; C03). The asymmetry parameter $A$ has been shown to be sensitive mainly to galaxy interactions and mergers but is also influenced by SF clumps, dust lanes, and projection effects (C03;

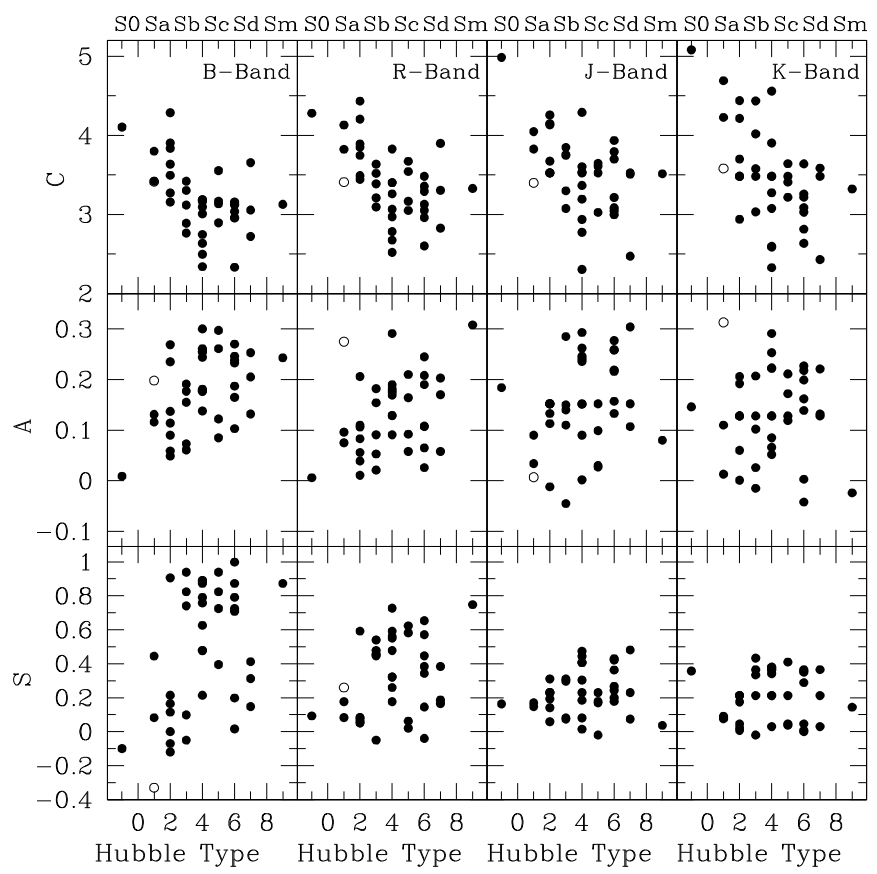

Fig. 11. $C A S$ parameters in the $B, R, J$ and $K$ bands versus the Hubble type. Galaxies with inclination higher than $80^{\circ}$ are shown with (open) circles.

Hernández-Toledo et al. 2005). The quantitative measure of $A$ in the present sample of isolated galaxies roughly spans the range $0.03 \leq A(R) \leq 0.23$, the average and standard deviation being $\langle A(R)\rangle=0.13 \pm 0.08$. The later types are slightly more asymmetric, on average, than the earlier types. The asymmetries reported here are definitively lower than those typical of interacting disk galaxies (see Fig. 10). The $A$-parameter decreases as the passband is redder (Fig. 9). Lower asymmetry values are in part related to SF effects; reflecting the sensitivity of $A$ to the passband.

\subsubsection{Clumpiness}

For our sample of isolated galaxies, $S(R)$ ranges roughly from 0.0 to 0.6 , the average and standard deviation values being $\langle S(R)\rangle=0.31 \pm 0.23$. The $S$-parameter is, on average, higher and more scattered in later types than in earlier types, as seen in Fig. 9 (see also below). Late-type galaxies present more current SF activity than early-type galaxies. Although the parameter $S$ in our isolated galaxies is typically lower than in interacting spirals, the differences are actually small and not as significant as in the case of the asymmetry parameter (Fig. 10).

\subsubsection{Correlations}

Figures 11 and 12 show the $B$-, $R$-, $J$ - and $K$ - band $C A S$ parameters versus morphological type $T$ and corrected total $(B-I)$ color. Nearly edge-on galaxies (inclination $\geq 80^{\circ}$ ) are plotted with open circles.

From Figs. 11 and 12 the trend of the $C$ and $A$ parameters with $T$ and total $(B-I)$ color typically tends to be more robust in the redder bands, emphasizing the merits of IR wavelengths, which are less contaminated from (transient) SF effects and better represent the basic structure of galaxies. The images from the 2MASS have a noisy background, especially in the $K$-band. 


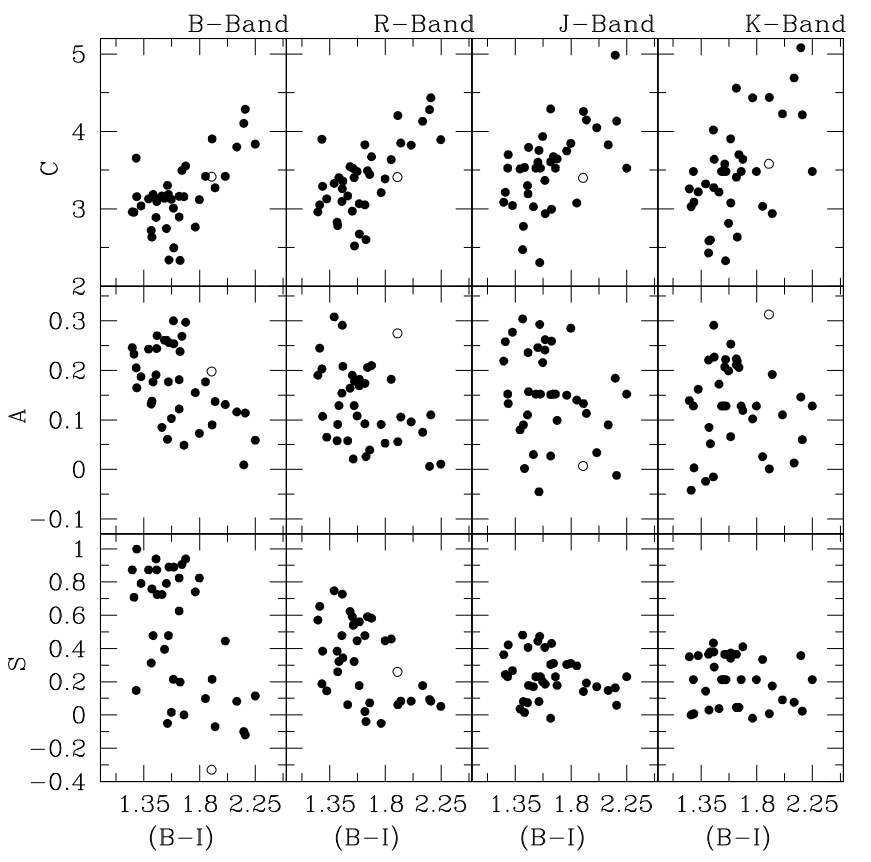

Fig. 12. $C A S$ parameters in the $B, R, J$ and $K$ bands versus the corrected total $(B-I)$ color. Galaxies with inclination higher than $80^{\circ}$ are shown with (open) circles.

Therefore, the $K$-band data discussed here should be taken only as indicative.

The morphological type is led mainly by the bulge-to-disk ratio. The global color is also affected by this ratio. Therefore, it is expected that earlier types will be more concentrated and redder. However, the $C$-parameter and the global color are not too sensitive to the bulge-to-disk ratio for galaxies with intermediate-to-low values of this ratio (say, Sb types and later); therefore, in these cases, the measured $C$ and global color reflect mostly the pure disk concentration and color. Thus, that $C$ depends on $T$ for late types mainly implies a connection between the spiral arm properties and the disk concentration. The dependence of $C$ on color would mainly imply that less concentrated disks have a more constant SF history, probably because their gas surface densities are low.

Concerning asymmetry, the observed dependence on $T$ indicates that most of the asymmetry of our isolated spirals is associated with the natural flocculency in later type galaxies, as well as with SF, which is more active for later types (as it is also evidenced by the trend of higher $S$-values as the types are later and the colors bluer). In this interpretation, the effect of large-scale perturbations (cf. interactions) is neglected.

Finally Fig. 13 shows a plot of the $C A S$ parameters vs. the tidal strength $Q$ parameter (as given in Verley et al. 2007). Galaxies with inclination higher than $80^{\circ}$ are shown with open circles. The disturbed candidates found in this study are shown as open-star symbols. Since values of $Q$ higher than -2 are indicative of galaxies being in interacting states (Athanassoula 1984), it is reasonable to expect some visible signs of morphological distortions in their optical images. Figure 13 shows no trend of $Q$ with any of the $C A S$ parameters.

Among our 9 morphologically distorted candidates with available $Q$, even those with a $Q>-2$ do not show a significantly higher $A$ or $S$ values, confirming that the $C A S$ parameters are not sensitive enough for detecting low intensity distortions.

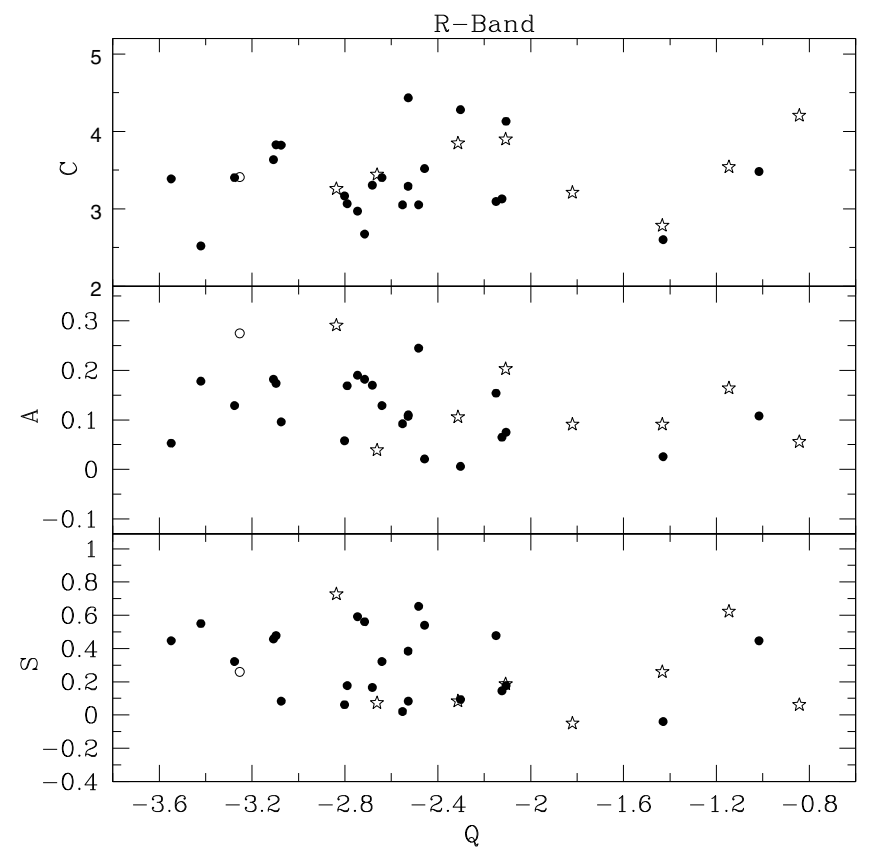

Fig. 13. $C A S$ parameters in the $R$ band versus tidal strength $Q$ (as reported by Verheijen 1997). Galaxies with inclination higher than $80^{\circ}$ are shown with open circles, disturbed candidates are shown with open stars.

\section{Summary and conclusions}

We present results of our BVRI CCD photometry for a set of 40 isolated galaxies selected from the CIG (Karachentseva 1973). We have shown that our derived parameters are generally in good agreement with the aperture photometry reported in the HyperLeda database and other individual photometric works. In addition, we present multi-aperture photometry (Appendix A) in order to facilitate further comparisons and contribute to the existing databases of aperture photometry (e.g., HyperLeda).

We have reevaluated the morphology of each of the galaxies based on our mosaic $R$-band and filter-enhanced $R$-band images, two-dimensional $(B-I)$ color maps, composed NIR JHK images from the 2MASS archives, photometric $\epsilon$ and PA radial profiles, and $R G B$ images from SDSS. Emphasis on structural features such as bars, rings and global disturbances was given.

We have demonstrated the convenience of analyzing the morphology through the combination of optical, near-infrared and SDSS RGB images.

The sample morphological types range from $\mathrm{Sa}$ to $\mathrm{Sm}$, $37.5 \%$ of the galaxies being SaSb, and $62.5 \%$ being of Sbc or later types. After our reclassification, we found that $\sim 40 \%$ of the galaxies are clearly barred (SB), while $\sim 25 \%$ more show some evidence of a suspected bar (SAB). The bar fraction is $68 \%$ for late-types and $60 \%$ for early-types. The average and standard deviation values of the $R$-band de-projected maximum ellipticity of the bars, $\epsilon_{\max }$, is $0.35 \pm 0.1$ for both early and late types, indicating that there is no trend of $\epsilon_{\max }$ with the morphological type. We have also found that $57.5 \%$ of the isolated galaxies in our sample show ring structures.

From our image analysis we found 9 candidates of isolated galaxies (CIG 539, 631, 744, 109, 135, 72, 716, 812, 838) with disturbed morphology. After reviewing those galaxies for the presence of equal and smaller size galaxies in their neighborhood (cf. Verley et al. 2007), we found CIG 744 having 
$M_{\mathrm{HI}} / L_{B}>1$ as a possible candidate to interact with a gasrich dark object. However, after studying the HI mass-to-light ratio as a function of disk central surface brightness for our 40 CIG galaxies, CIG 744 is interpreted rather as an intrinsically gas-rich but possibly less evolved system, instead.

We have also calculated the $B V R I-, J, H$ and $K$-band $C A S$ parameters for the sample. The $C A S$ averages and standard deviations in the $R$-band for the $\mathrm{SaSb}$ and $\mathrm{SbcSm}$ subsamples are: $\langle C(R)\rangle(\mathrm{SaSb})=3.74 \pm 0.40,\langle A(R)\rangle(\mathrm{SaSb})=0.09 \pm 0.06$, $\langle S(R)\rangle(\mathrm{SaSb})=0.23 \pm 0.21$ and $\langle C(R)\rangle(\mathrm{SbcSm})=3.19 \pm 0.36$, $\langle A(R)\rangle(\mathrm{SbcSm})=0.16 \pm 0.07,\langle S(R)\rangle(\mathrm{SbcSm})=0.38 \pm 0.23$, respectively. These values are in good agreement with previous results for a similarly selected subsample of isolated galaxies and other studies of non-interacting galaxies. The results of the $S$ parameter should be taken only as indicative due to the noisy nature of the $J, H$ and $K$ images.

While $C$ systematically increases from bluer to redder bands, both $A$ and $S$ decrease. The $C$ and $A$ parameters present more robust trends with the morphological type $T$ and the total $(B-$ I) color in the redder bands, suggesting that the basic structure of galaxies is revealed better towards the infrared bands. The $C$-parameter tends to be higher for earlier type and redder galaxies, while $A$ and $S$ tend to be lower.

Through the loci of our isolated galaxies in the projected planes of the $C A S$ space, we have tested the ability of these diagrams to differentiate between isolated and interacting/starburst spirals. The observed values of the CAS parameters for these isolated galaxies are always lower than the expected values for interacting galaxies. The distortions seen in our nine morphologically distorted candidates are not strong enough to be detected by the $C A S$ parameters.

This paper is a step in the building of a well-defined sample of local isolated galaxies with uniform and detailed photometric information. This is relevant because it provides a fair database for comparison with model predictions, as well as with observed samples of galaxies in other environments and at higher redshifts.

Through our image procedures we were able to detect a set of morphologically distorted galaxy candidates. However, a more careful and detailed analysis involving our surface photometry and other physical properties is required. A study like this will provide better constraints on the end product of galaxy-galaxy merging and how this is changing the morphological properties of the isolated galaxy population.

Acknowledgements. H.M.H.T. thanks the staff of the Observatorio Astronómico Nacional in San Pedro Mártir, Baja California, for the help with the observation runs. This work was funded by CONACyT grant 42810 to H.M.H.T. This research has made use of the NASA/IPAC Extragalactic Database (NED) which is operated by the Jet Propulsion Laboratory, California Institute of Technology, under contract with the National Aeronautics and Space Administration. We acknowledge use of the HyperLeda database (http:// leda.univ-lyon 1.fr). This publication makes use of data products from the Two Micron All Sky Survey, which is a joint project of the University of Massachusetts and the Infrared Processing and Analysis Center, California Institute of Technology, funded by the National Aeronautics and Space Administration and the National Science Foundation. Funding for the Sloan Digital Sky Survey (SDSS) has been provided by the Alfred P. Sloan Foundation, the Participating Institutions, the National Aeronautics and Space Administration, the National Science Foundation, the US Department of Energy, the Japanese Monbukagakusho, and the Max Planck Society. The SDSS Web site is http://www.sdss.org/. The SDSS is managed by the Astrophysical Research Consortium (ARC) for the Participating Institutions. The Participating Institutions are The University of Chicago, Fermilab, the Institute for Advanced Study, the Japan Participation Group, The Johns Hopkins University, Los Alamos National Laboratory, the Max-Planck-Institute for Astronomy (MPIA), the Max-Planck-Institute for Astrophysics (MPA), New
Mexico State University, University of Pittsburgh, Princeton University, the United States Naval Observatory, and the University of Washington. S.O.E. thanks CONACyT through grant 42810 for the financial support during the preparation of this paper.

\section{References}

Abraham, R. G., Tanvir, N. R., Santiago, B. X., et al. 1996, MNRAS, 279, L47 Athanassoula, E. 1984, Phys. Rep., 114, 321

Athanassoula, E., \& Misiriotis, A. 2002, MNRAS, 330, 35

Avila-Reese, V. 2006, preprint [arXiv: astro-ph/0605212]

Avila-Reese, V., \& Firmani, C. 2000, RMxAA, 36, 23A

Avila-Reese, V., Carrillo, A., Valenzuela, O., \& Klypin, A. 2005, MNRAS, 361, 997

Avila-Reese, V., Zavala, J., Firmani, C., \& Hernández-Toledo, H. 2008, AJ, submitted

Berentzen, I., Shlosman, I., \& Jogee, S. 2006, ApJ, 637, 582

Bershady, M. A., Jangren, A., \& Conselice, C. J. 2000, AJ, 119, 2645

Buta, R. 1986, ApJS, 61, 609

Buta, R. 1995, ApJS, 96, 39

Chevalier, C., \& Ilovaisky, S. A. 1991, A\&A, 90, 225

Conselice, C. J. 1997, PASP, 109, 1251

Conselice, C. J. 2003, ApJS, 147, 1 (C03)

Debattista, V. P., \& Sellwood, J. A. 2000, ApJ, 543, 704

de Jong, R. S. 1996, A\&A, 313, 377

Dressler, A. 1980, ApJ, 236, 351

Eskridge, P. B., Frogel, J. A., Pogge, R. W., et al. 2000, AJ, 119, 536

Firmani, C., \& Avila-Reese, V. 2000, MNRAS, 315, 457

Frei, Z., Guhathakurta, P., Gunn, J. E., \& Tyson, J. A. 1996, AJ, 111, 174

Giovanelli, R., Haynes, M. P., Salzer, J. J., et al. 1995, AJ, 110, 1059

Hernández-Toledo, H. M., Dultzin-Hacyan, D., González, J. J., \& Sulentic, J. 1999, AJ, 118, 108

Hernández-Toledo, H. M., Avila-Reese, V., Conselice, C. J., \& Puerari, I. 2005, AJ, 129, 682

Hernández-Toledo, H. M., Zendejas-Dominguez, J., \& Avila-Reese, V. 2007, AJ, 135,2286

Karachentsev, I. D., Karachentseva, V. E., \& Huchtmeier, W. K. 2006, A\&A, 451,817

Karachentsev, I. D., Karachentseva, V. E., \& Huchtmeier, W. K. 2007, ed. D. I. Makarov, \& S. S. Kaisin [arXiv: astro-ph/0708.1046v1]

Karachentseva, V. E. 1973, Astrof. Issledovanija Byu. Spec. Ast. Obs., 8, 3

Klypin, A. A., Kravtsov, A. V., Valenzuela, O., \& Prada, F. 1999, ApJ, 522, 82 Landolt, A. U. 1992, AJ, 104, 340L

Laurikainen, E., Salo, H., \& Rautiainen, P. 2002, MNRAS, 331, 880

McGaugh, S. S., \& de Blok, W. J. G. 1997, ApJ, 481, 689

Moore, B., Ghigna, S., Governato, F., et al. 1999, ApJ, 524, L19

Morgan, W. W., \& Osterbrock, D. E. 1969, AJ, 74, 515

Park, C., Gott, J. R., \& Choi, Y. [arXiv:0708.4118]

Pisano, D. J., Wilcots, E. M., \& Liu, C. T. 2002, ApJS, 142, 161

Postman, M., \& Geller, M. J. 1984, ApJ, 281, 95

Prugniel, P. 1987, CDS Bull., 33, 17

Richter, O., \& Sancisi, R. 1994, A\&A, 290, L9

Rix, H., \& Zaritsky, D. 1995, ApJ, 447, 82

Roberts, M. S., \& Haynes, M. P. 1994, ARA\&A, 32, 115

Schlegel, D. J., Finkbeiner, D. P., \& Davis, M. 1998 ApJ, 500, 525

Shustov, B., Wiebe, D., \& Tutukov, A. 1997, A\&A, 317, 397

Skrutskie, M. F., Cutri, R. M., Stiening, R., et al. 2006, AJ, 131, 1163

Sofue, Y. 1993, PASP, 105, 308

Sulentic, J. W., Verdes-Montenegro, L., Bergond, G., et al. 2006, A\&A, 449, 937

Trentham, N., Möller, O., \& Ramirez-Ruiz, E. 2001, MNRAS, 322, 658

Tully, R. B. 2005, ApJ, 618, 214

Tully, R. B., \& Pierce, M. J. 2000, ApJ, 533, 744

Tully, R. B., Pierce, M. J., Huang, J., et al. 1998, AJ, 115, 2264

Valenzuela, O., \& Klypin, A. 2003, MNRAS, 345, 406

van den Bosch, F., Yang, X., \& Mo, H. J. MNRAS, 340, 771

Verheijen, M. A. W. 1997, Ph.D. Thesis,

Verley, S., Leon, S., Verdes-Montenegro, L., et al. 2007, A\&A, 472, 121

Wang, B., \& Heckman, T. M. 1996, ApJ, 457, 645

Whitford, A. E. 1936, ApJ, 83, 424

Wozniak, H., Friedli, D., Martinet, L., Martin, P., \& Bratschi, P. 1995, A\&AS, 111, 115

Yang, X., Mo, H. J., \& van den Bosch, F. C. 2005, MNRAS, 358, 217

Zavala, J., Avila-Reese, V., Hernández-Toledo, H., \& Firmani, C. 2003, A\&A, 412, 633 


\section{Appendix A: Aperture photometry}

Since the birth of galaxy photometry (Whitford 1936), the amount of photometric data has increased exponentially (Prugniel 1987). However, these data are inhomogeneous in both quality and format: photographic, photoelectric, or more recently, CCD observations. The data are usually presented as centered aperture photometry through circular or elliptical apertures or as photometric profiles. In order to take into account the continuously growing amount of photometric data and at the same time make different photometric data reports somehow comparable, we present in Table A.1 our estimations of integrated magnitudes in two additional concentric circular apertures. Column (1) is the original CIG number, Cols. (2) and (7) give the logarithm of the aperture radius (in units of $0.1^{\prime}$, see the HyperLeda convention) for each isolated spiral galaxy. Columns (3)-(6) and (8)-(11) give their corresponding magnitudes in the $B, V$, $R$, and $I$ bands. Typical uncertainties in the magnitudes are 0.11 , $0.12,0.11$, and 0.12 in $B, V, R$, and $I$ bands, respectively. 
H. M. Hernández-Toledo and S. Ortega-Esbrí: Broad-band BVRI photometry of isolated spiral galaxies, Online Material p 2

Table A.1. Magnitudes at two different circular apertures. $A 2>A 1$.

\begin{tabular}{|c|c|c|c|c|c|c|c|c|c|c|}
\hline$\overline{\mathrm{CIG}}$ & $\overline{\log A 2}$ & $\overline{\bar{B}}$ & $\overline{\bar{V}}$ & $\overline{\bar{R}}$ & $\overline{I I}$ & $\overline{\log A 1}$ & $\overline{\bar{B}}$ & $\bar{V}$ & $\bar{R}$ & $I$ \\
\hline CIG 72 & 1.00518 & 14.513 & 13.692 & 13.163 & 12.462 & 0.70415 & 15.235 & 14.339 & 13.781 & 13.071 \\
\hline CIG 89 & 1.209 & 12.475 & 11.530 & 10.962 & 10.026 & 0.908 & 12.956 & 11.951 & 11.386 & 10.520 \\
\hline CIG 103 & 1.32045 & 13.497 & 12.564 & 11.932 & 11.161 & 1.01942 & 14.245 & 13.257 & 12.613 & 11.800 \\
\hline CIG 109 & 1.20016 & 12.734 & 12.037 & 11.563 & 10.869 & 0.89913 & 13.091 & 12.446 & 11.958 & 11.302 \\
\hline CIG 135 & 1.04657 & 14.701 & 13.797 & 13.223 & 12.443 & 0.74554 & 15.168 & 14.219 & 13.615 & 12.840 \\
\hline CIG 145 & 1.10784 & 15.046 & 13.919 & 13.264 & 12.404 & 0.80681 & 15.329 & 14.194 & 13.531 & 12.671 \\
\hline CIG 156 & 0.92600 & 13.677 & 13.064 & 12.631 & 11.986 & 0.62497 & 14.150 & 13.564 & 13.172 & 12.609 \\
\hline CIG 191 & 1.04657 & 14.564 & 13.546 & 13.117 & 12.060 & 0.74554 & 14.904 & 13.873 & 13.298 & 12.419 \\
\hline CIG 199 & 1.15131 & 14.519 & 13.921 & 13.451 & 12.796 & 0.85028 & 14.908 & 14.247 & 13.754 & 13.102 \\
\hline CIG 309 & 1.227 & 11.644 & 10.698 & 10.143 & 9.249 & 0.926 & 12.240 & 11.259 & 10.711 & 9.855 \\
\hline CIG 314 & 1.415 & 12.331 & 11.749 & 11.344 & ; & 1.114 & 12.788 & 12.143 & 11.716 & \\
\hline CIG 392 & 0.92600 & 14.997 & 14.132 & 13.592 & 12.892 & 0.62497 & 15.290 & 14.428 & 13.876 & 13.197 \\
\hline CIG 448 & 1.30621 & 12.631 & 12.011 & 11.518 & 10.849 & 1.00518 & 12.904 & 12.260 & 11.749 & 11.078 \\
\hline CIG 477 & 1.13012 & 12.619 & 11.953 & 11.412 & 10.775 & 0.82909 & 12.986 & 12.297 & 11.745 & 11.111 \\
\hline CIG 491 & 0.88979 & 14.706 & 14.002 & 13.489 & 12.852 & 0.58876 & 15.243 & 14.484 & 13.936 & 13.296 \\
\hline CIG 500 & 1.09626 & 14.158 & 13.247 & 12.689 & 12.018 & 0.79523 & 14.940 & 13.954 & 13.371 & 12.676 \\
\hline CIG 507 & 1.13012 & 15.334 & 14.762 & 14.287 & 13.609 & 0.82909 & 15.674 & 15.086 & 14.639 & 14.083 \\
\hline CIG 512 & 1.25234 & 14.001 & 13.251 & 12.748 & 12.141 & 0.95131 & 15.104 & 14.203 & 13.662 & 13.041 \\
\hline CIG 525 & 1.07213 & 14.033 & 13.332 & 12.817 & 12.188 & 0.77110 & 14.689 & 13.892 & 13.343 & 12.689 \\
\hline CIG 539 & 1.07213 & 14.211 & 13.353 & 12.808 & 12.122 & 0.77110 & 14.568 & 13.660 & 13.092 & 12.406 \\
\hline CIG 547 & 1.22703 & 13.661 & 13.056 & 12.640 & 12.127 & 0.92600 & 14.394 & 13.749 & 13.312 & 12.773 \\
\hline CIG 575 & 1.18127 & 13.262 & 12.623 & 12.149 & 11.569 & 0.88024 & 13.692 & 13.021 & 12.522 & 11.918 \\
\hline CIG 616 & 1.26842 & 14.400 & 13.268 & 12.675 & 11.918 & 0.96739 & 14.579 & 13.657 & 13.034 & 12.251 \\
\hline CIG 624 & 1.306 & 11.970 & 11.538 & 11.138 & 10.491 & 1.005 & 12.714 & 12.208 & 11.785 & 11.182 \\
\hline CIG 630 & 1.10784 & 14.157 & 13.476 & 12.981 & 12.406 & 0.80681 & 14.655 & 13.925 & 13.400 & 12.778 \\
\hline CIG 631 & 1.18127 & 13.207 & 12.962 & 12.475 & 11.599 & 0.88024 & 13.411 & 13.157 & 12.662 & 11.799 \\
\hline CIG 712 & 1.18127 & 13.313 & 12.512 & 11.964 & 11.294 & 0.88024 & 13.958 & 13.118 & 12.553 & 11.878 \\
\hline CIG 716 & 1.00518 & 15.023 & 14.125 & 13.561 & 12.879 & 0.70415 & 15.829 & 14.807 & 14.179 & 13.489 \\
\hline CIG 744 & 1.25234 & 14.672 & 14.137 & 13.798 & 13.298 & 0.95131 & 15.204 & 14.600 & 14.226 & 13.725 \\
\hline CIG 766 & 1.18127 & 12.327 & 11.770 & 11.339 & 10.795 & 0.88024 & 12.880 & 12.292 & 11.841 & 11.295 \\
\hline CIG 772 & 1.22703 & 13.925 & 13.217 & 12.715 & 12.121 & 0.92600 & 14.384 & 13.618 & 13.086 & 12.432 \\
\hline CIG 800 & 1.04657 & 14.322 & 13.515 & 12.950 & 12.285 & 0.74554 & 15.074 & 14.229 & 13.639 & 12.930 \\
\hline CIG 805 & 1.07213 & 14.389 & 13.628 & 13.098 & 12.484 & 0.77110 & 14.887 & 14.090 & 13.540 & 12.896 \\
\hline CIG 812 & 1.26842 & 13.229 & 12.450 & 11.880 & 11.177 & 0.96739 & 13.802 & 12.931 & 12.326 & 11.569 \\
\hline CIG 838 & 1.16153 & 14.120 & 13.347 & 12.825 & 12.246 & 0.86050 & 14.623 & 13.830 & 13.284 & 12.656 \\
\hline CIG 840 & 1.13012 & 13.726 & 13.001 & 12.488 & 11.907 & 0.82909 & 14.659 & 13.890 & 13.340 & 12.680 \\
\hline CIG 858 & 1.07213 & 14.412 & 13.533 & 12.954 & 12.256 & 0.77110 & 14.937 & 13.991 & 13.383 & 12.668 \\
\hline CIG 861 & 1.00518 & 15.454 & 14.712 & 14.204 & 13.624 & 0.70415 & 15.831 & 15.079 & 14.553 & 13.948 \\
\hline CIG 862 & 1.04657 & 14.047 & 13.331 & 12.792 & 12.321 & 0.74554 & 14.642 & 13.874 & 13.306 & 12.708 \\
\hline CIG 947 & 1.313 & 11.560 & 10.660 & $\ldots$ & $\ldots$ & 1.012 & 12.140 & 11.145 & $\ldots$ & $\ldots$ \\
\hline
\end{tabular}


H. M. Hernández-Toledo and S. Ortega-Esbrí: Broad-band BVRI photometry of isolated spiral galaxies, Online Material p 3 Appendix B 
H. M. Hernández-Toledo and S. Ortega-Esbrí: Broad-band BVRI photometry of isolated spiral galaxies, Online Material p 4
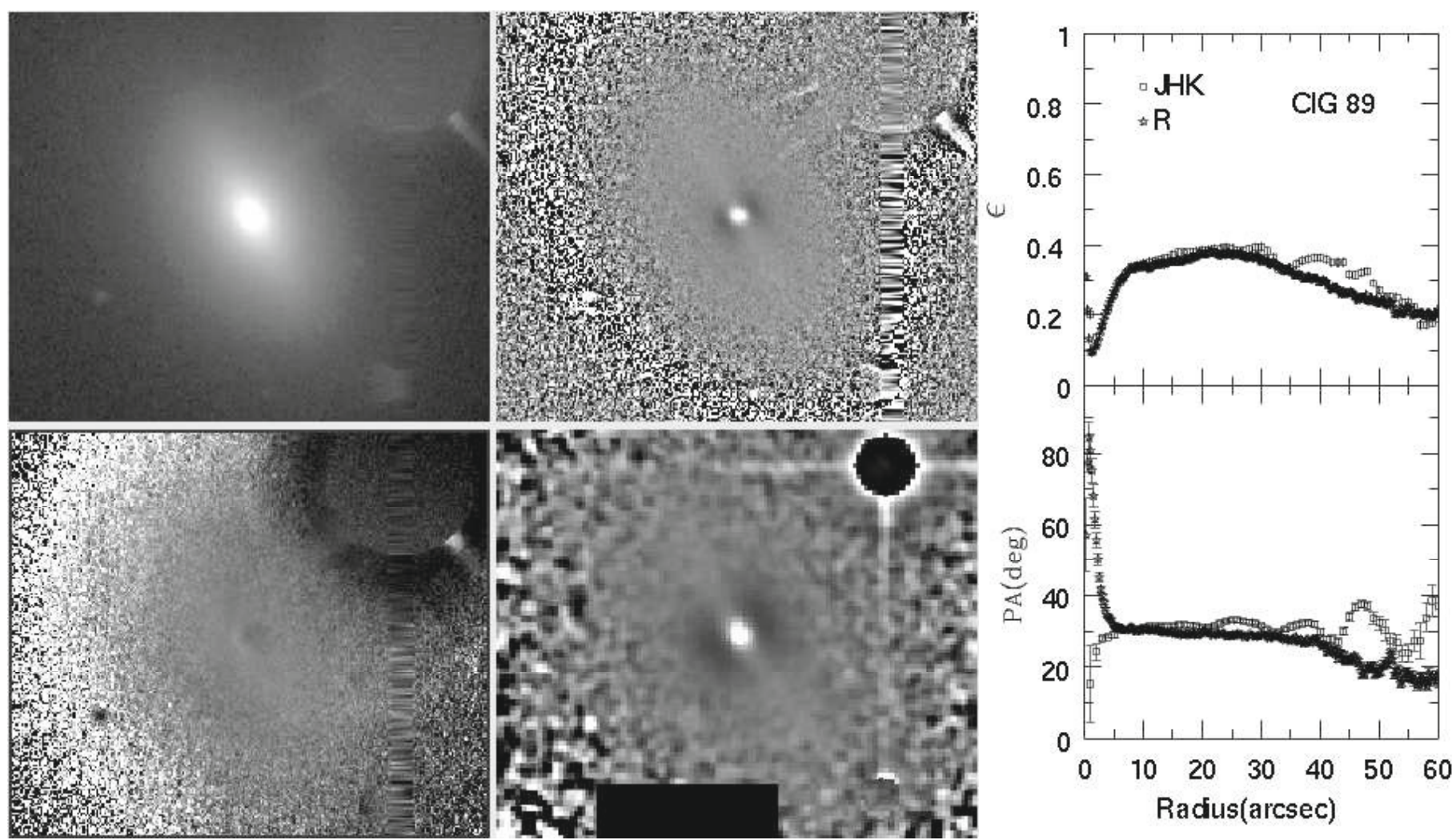

Fig. B.1. CIG 89 Mosaic. Same as Fig. 3. The major diameter of the galaxy in the optical images is 2.6 arcmin.
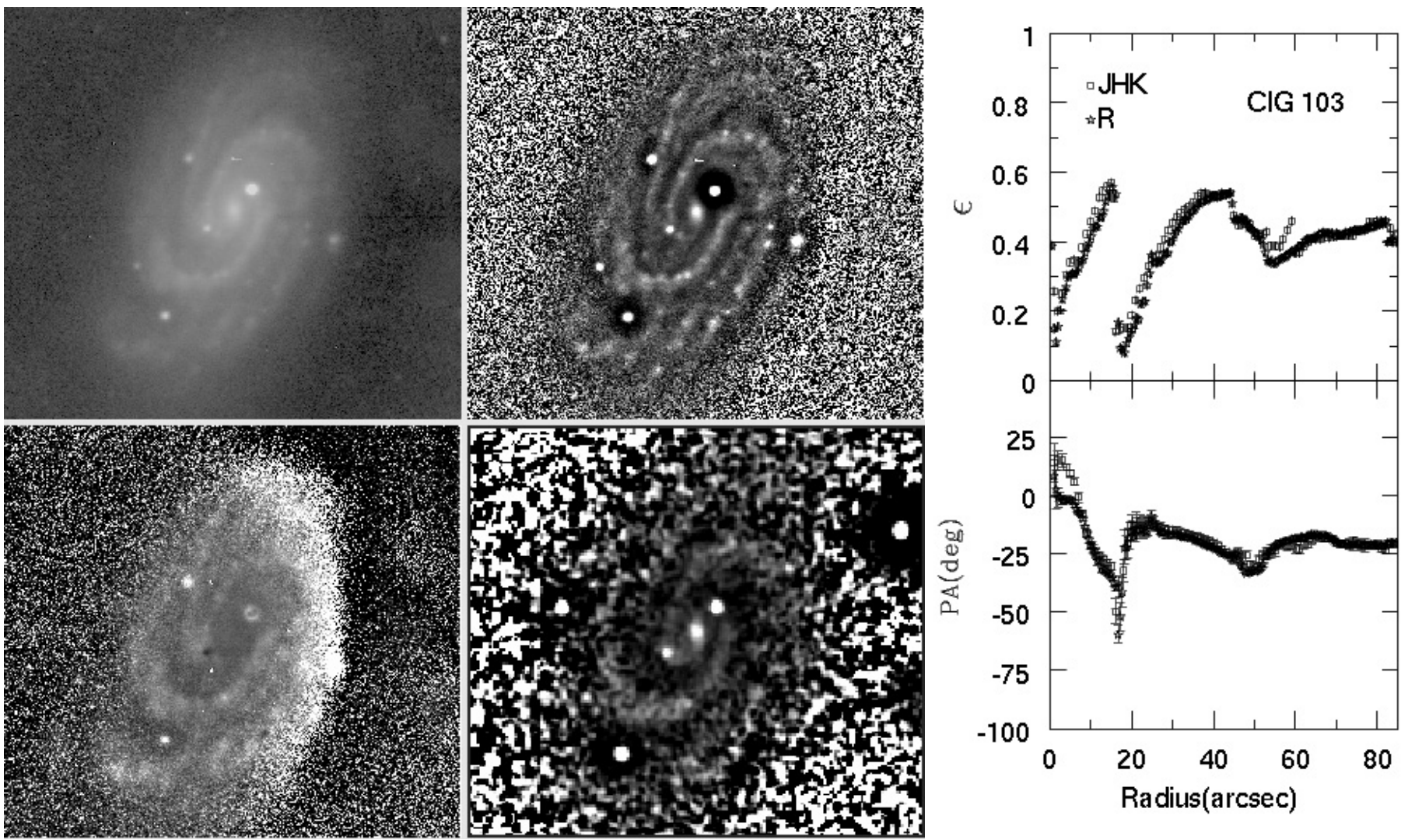

Fig. B.2. CIG 103 Mosaic. Same as Fig. 3. The major diameter of the galaxy in the optical images is $3.5 \operatorname{arcmin}$. 
H. M. Hernández-Toledo and S. Ortega-Esbrí: Broad-band BVRI photometry of isolated spiral galaxies, Online Material p 5
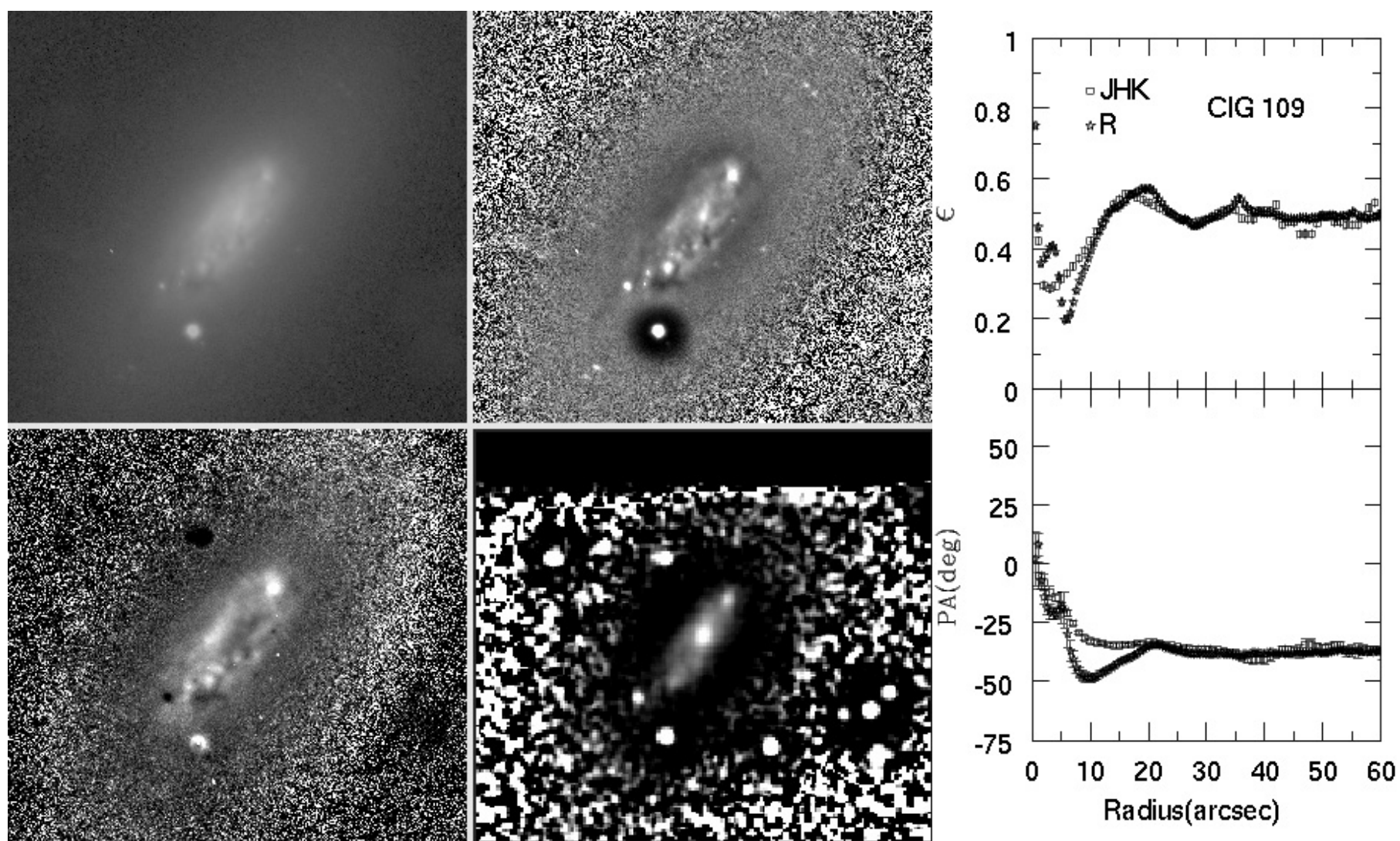

Fig. B.3. CIG 109 Mosaic. Same as Fig. 3. The major diameter of the galaxy in the optical images is 2.4 arcmin.
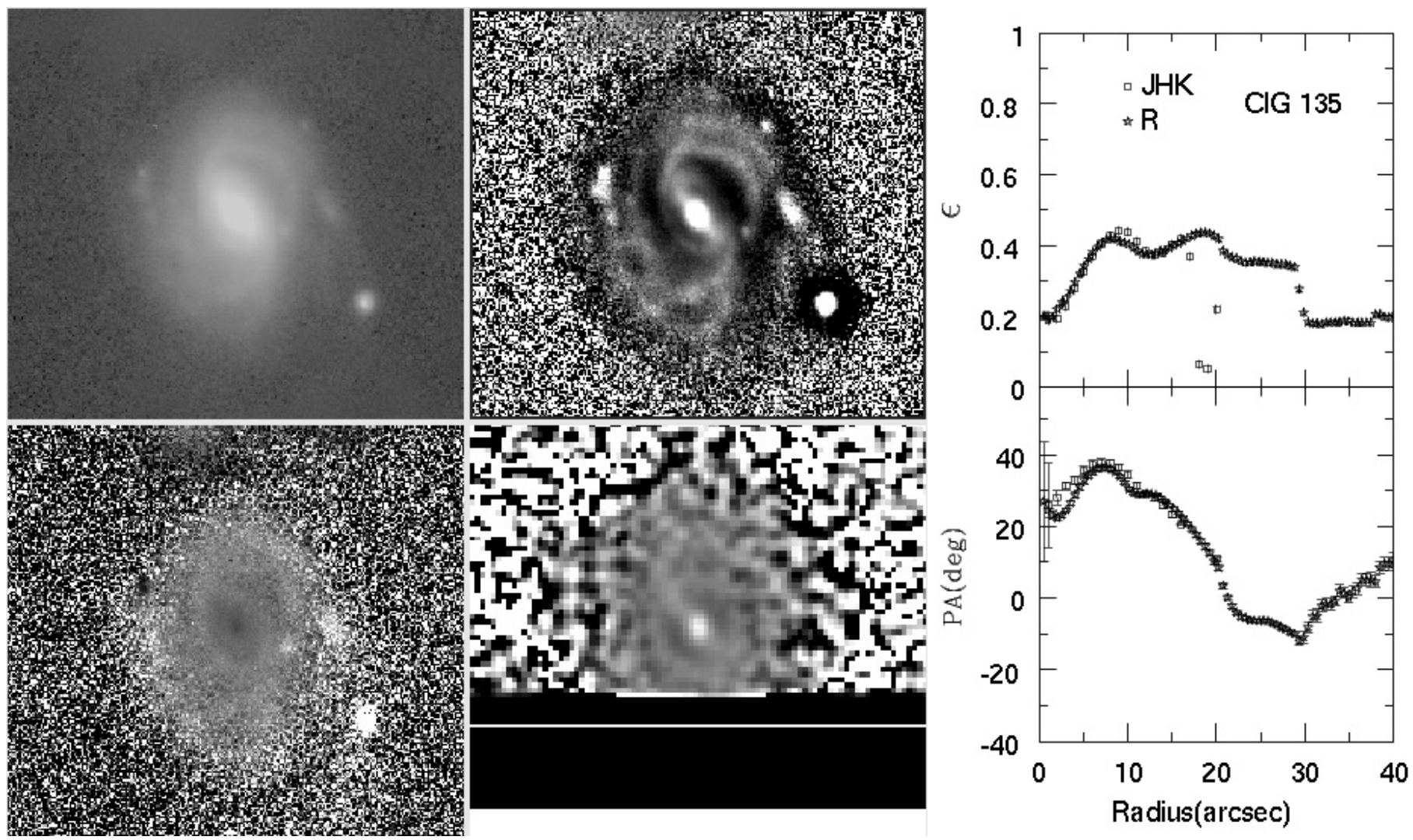

Fig. B.4. CIG 135 Mosaic. Same as Fig. 3. The major diameter of the galaxy in the optical images is $1.1 \mathrm{arcmin}$. 
H. M. Hernández-Toledo and S. Ortega-Esbrí: Broad-band BVRI photometry of isolated spiral galaxies, Online Material $p 6$
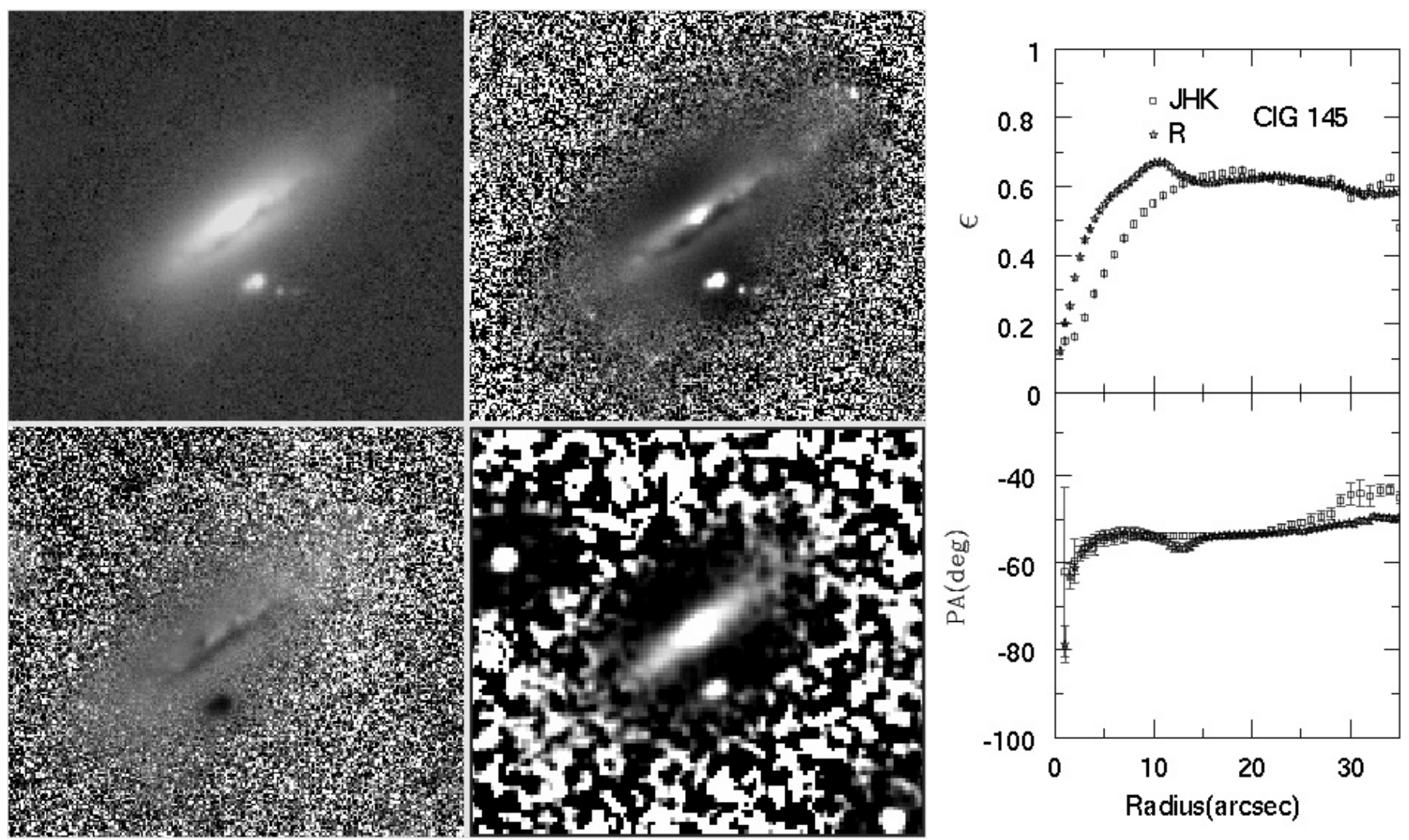

Fig. B.5. CIG 145 Mosaic. Same as Fig. 3. The major diameter of the galaxy in the optical images is $1.3 \mathrm{arcmin}$.
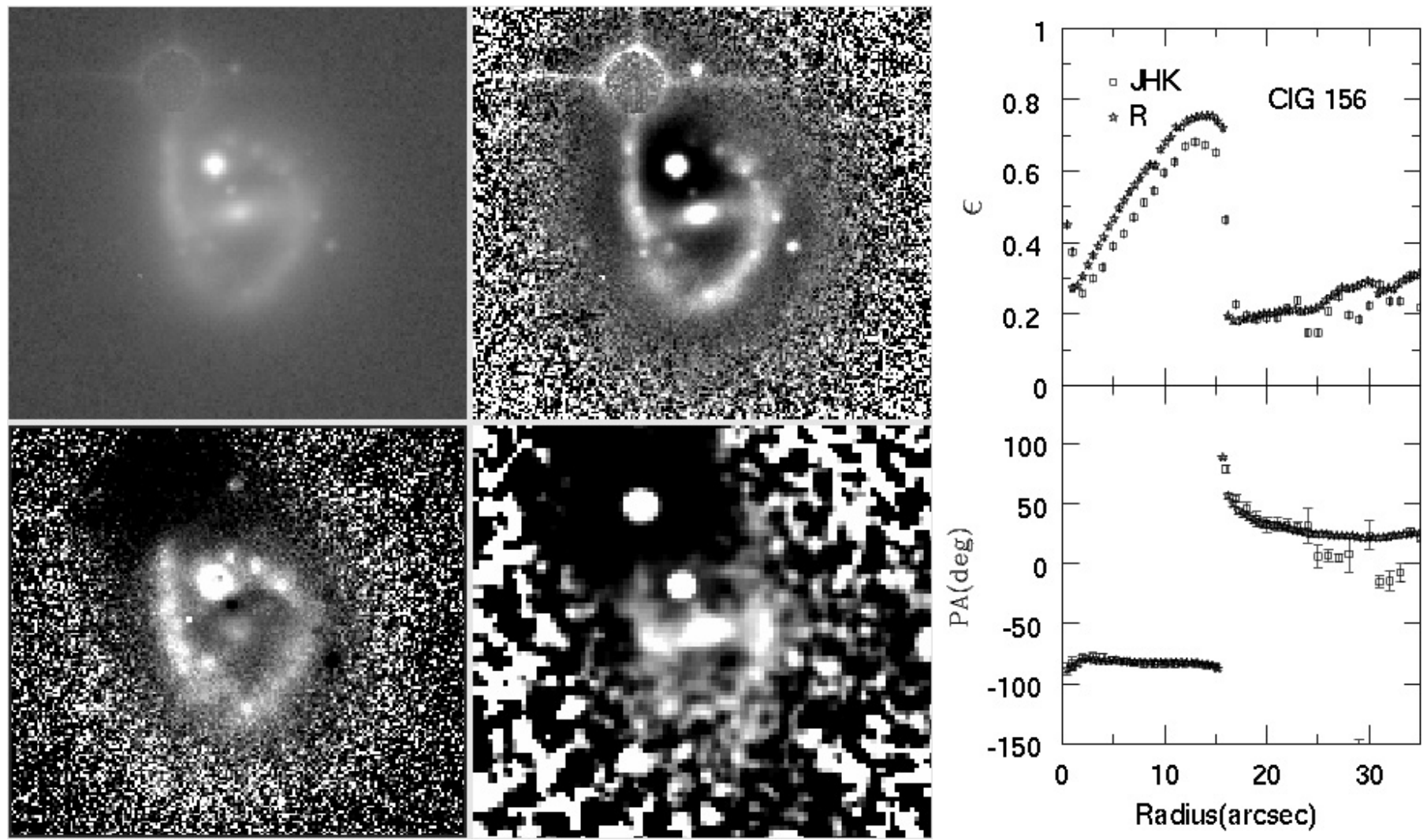

Fig. B.6. CIG 156 Mosaic. Same as Fig. 3. The major diameter of the galaxy in the optical images is 0.8 arcmin. 
H. M. Hernández-Toledo and S. Ortega-Esbrí: Broad-band BVRI photometry of isolated spiral galaxies, Online Material p 7
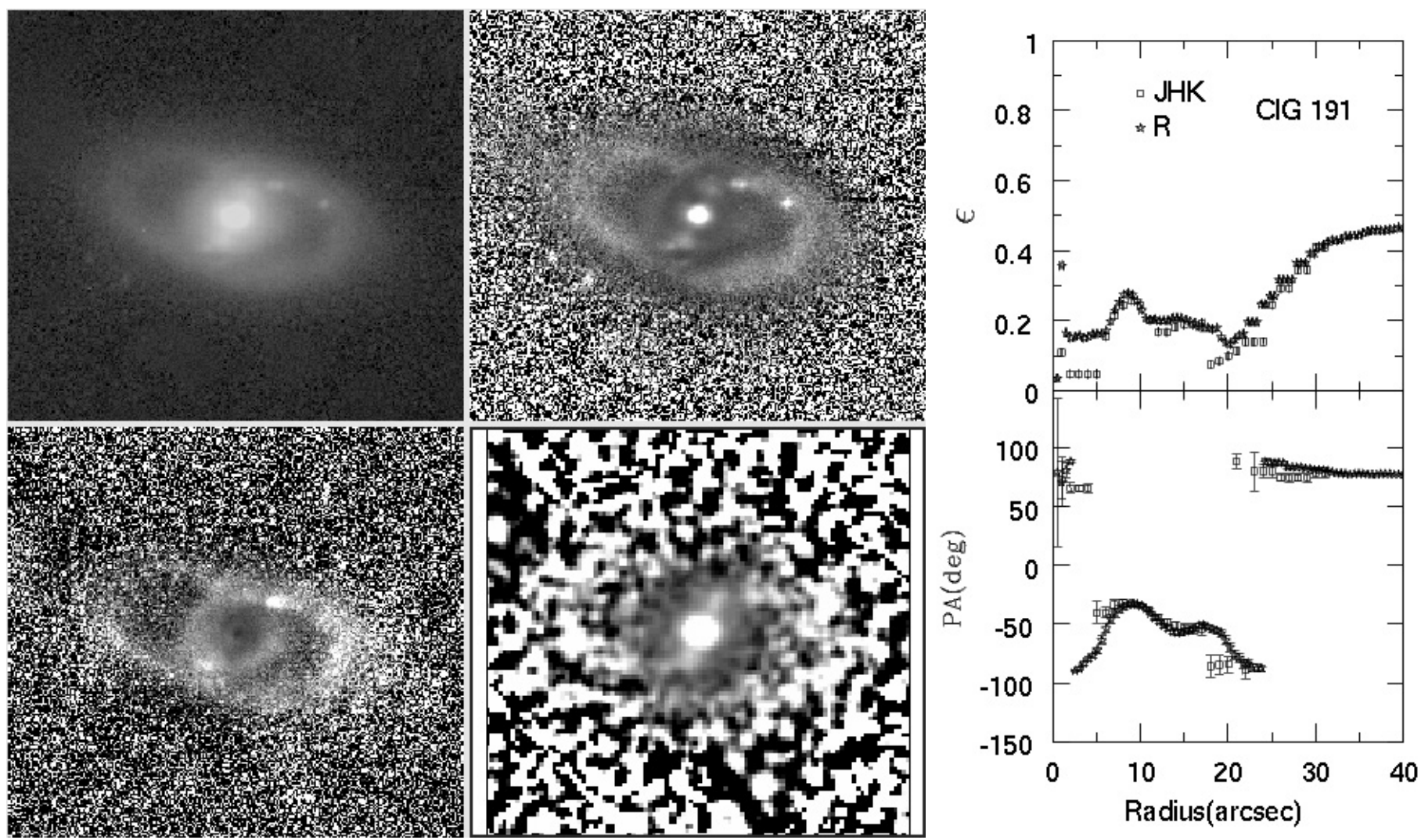

Fig. B.7. CIG 191 Mosaic. Same as Fig. 3. The major diameter of the galaxy in the optical images is $1.2 \mathrm{arcmin}$. 
H. M. Hernández-Toledo and S. Ortega-Esbrí: Broad-band BVRI photometry of isolated spiral galaxies, Online Material p 8
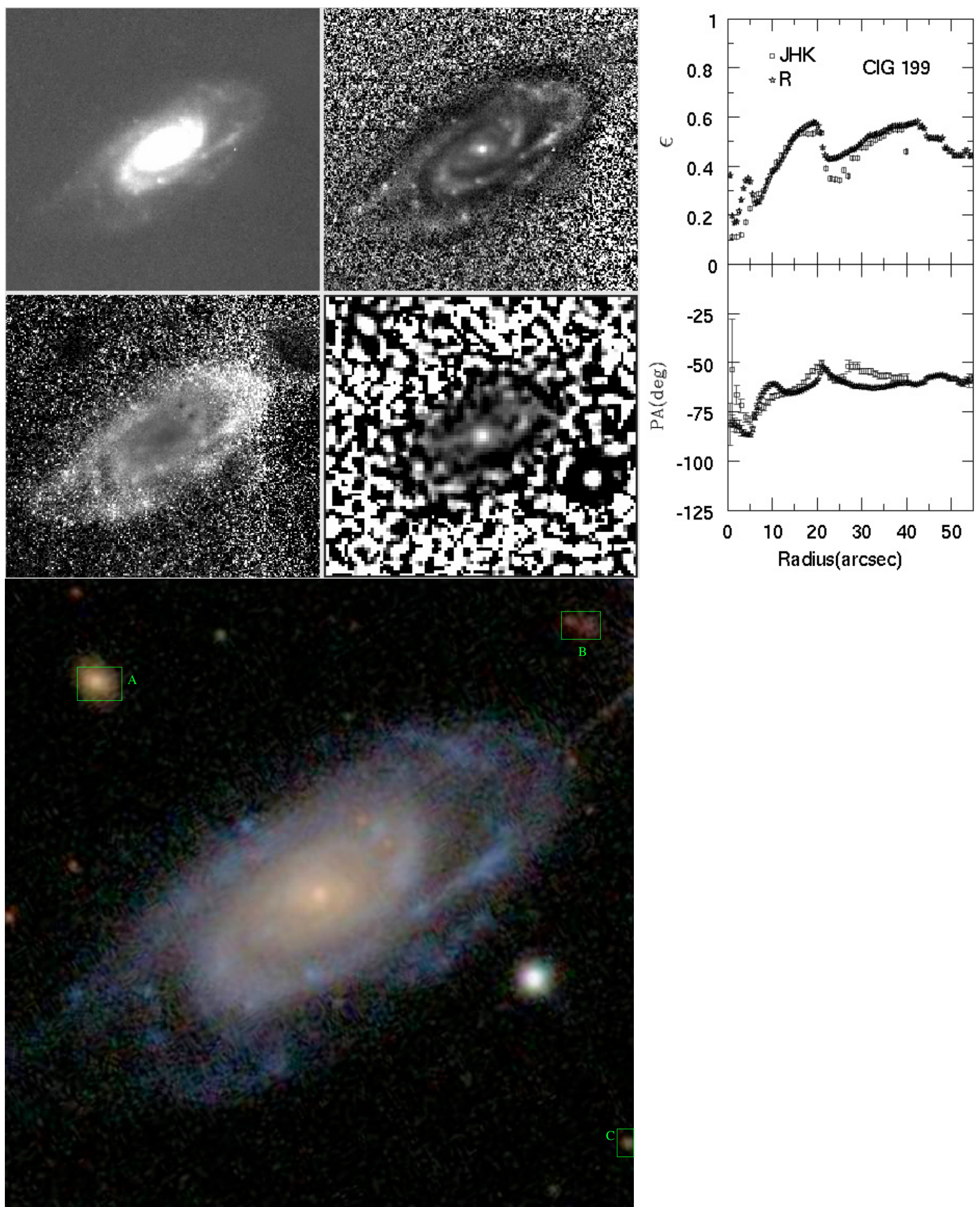

Fig. B.8. CIG 199 Mosaic. Same as Fig. 3. The major diameter of the galaxy in the optical images is $1.5 \mathrm{arcmin}$. 
H. M. Hernández-Toledo and S. Ortega-Esbrí: Broad-band BVRI photometry of isolated spiral galaxies, Online Material p 9


Fig. B.9. CIG 309 Mosaic. Same as Fig. 3. The major diameter of the galaxy in the optical images is $4.3 \mathrm{arcmin}$. 
H. M. Hernández-Toledo and S. Ortega-Esbrí: Broad-band BVRI photometry of isolated spiral galaxies, Online Material p 10
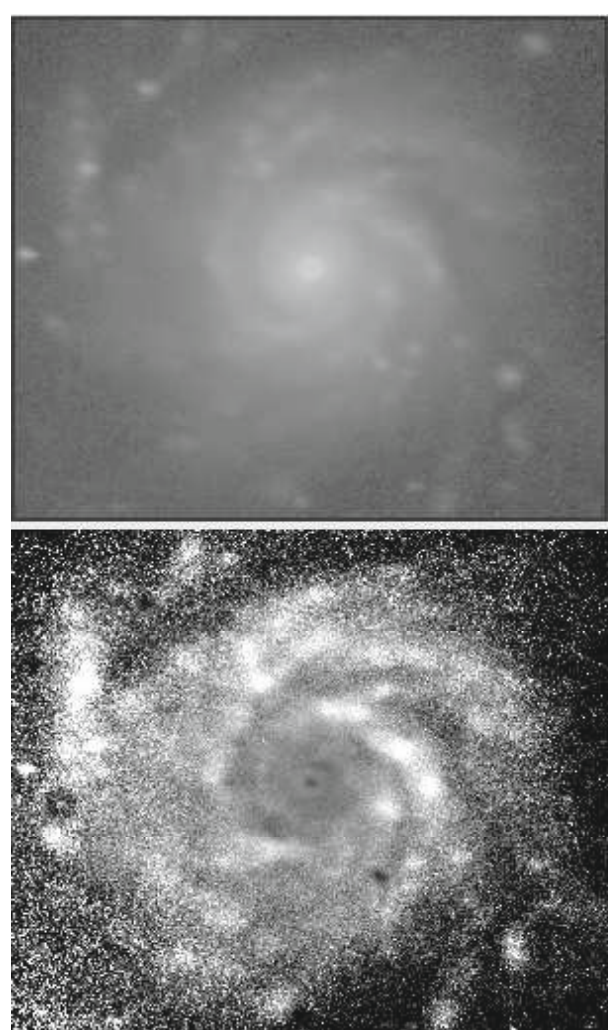
等
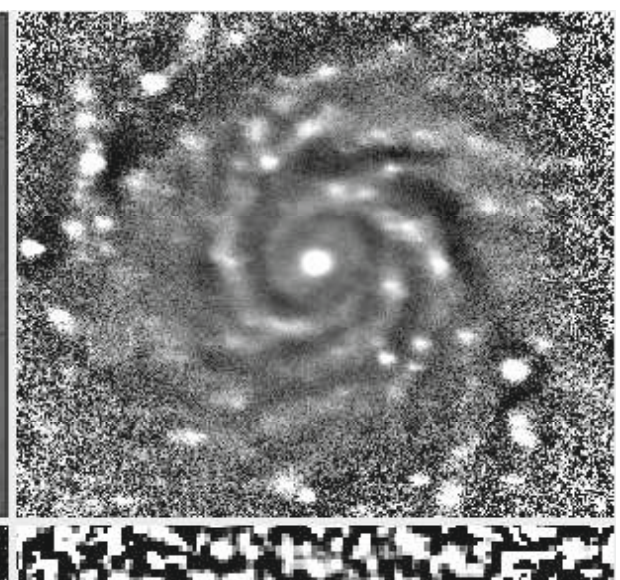

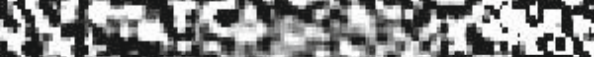
tow If6.

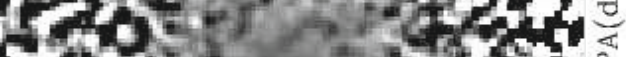
$3 t^{2}+25+2$

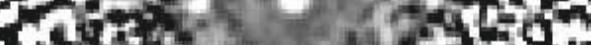

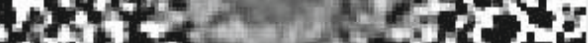

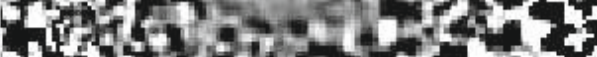

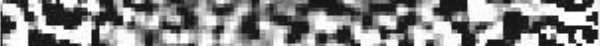
Q7) $2+35,+2 y-7,4$.

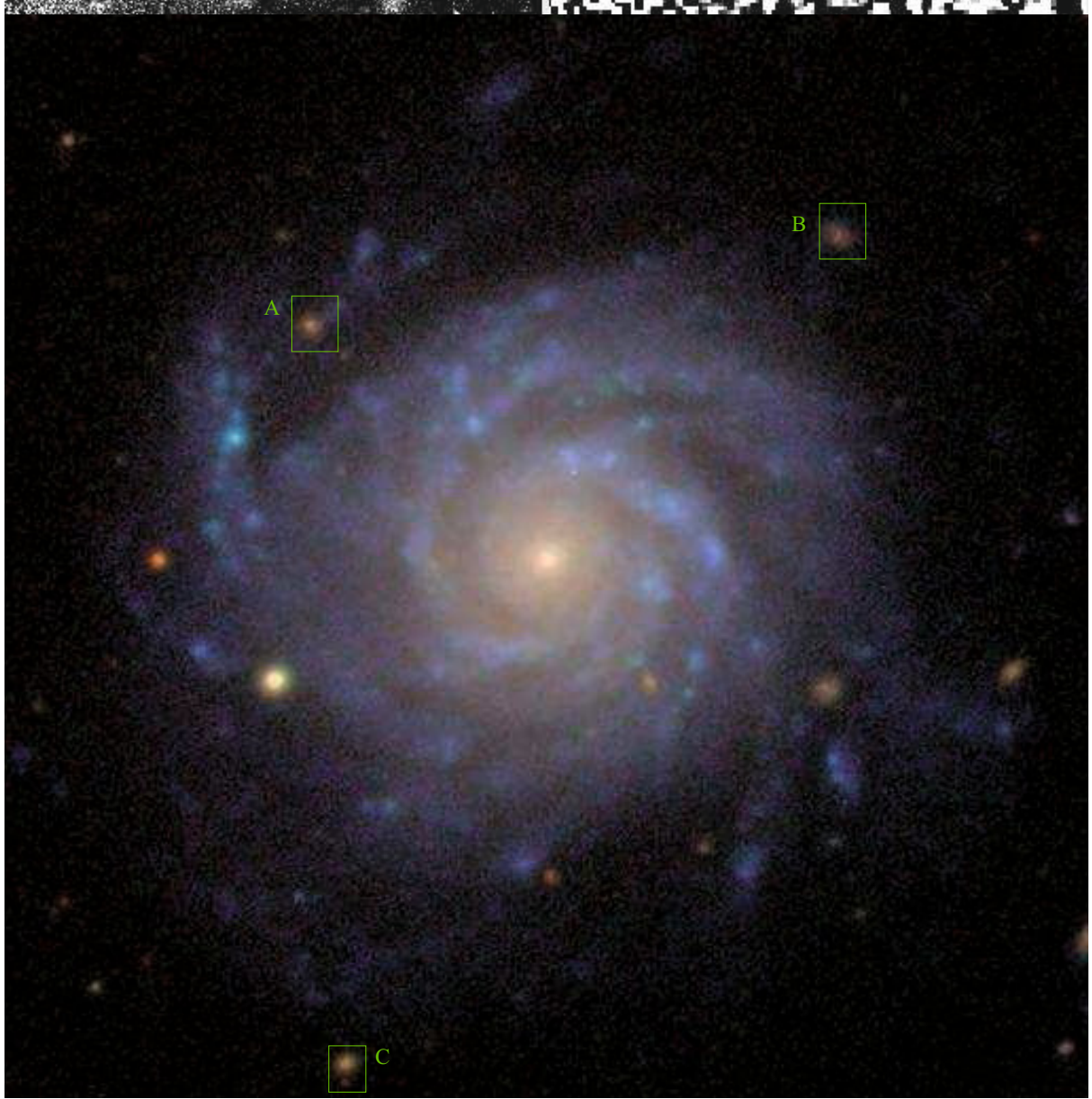

Fig. B.10. CIG 314 Mosaic. Same as Fig. 3. The major diameter of the galaxy in the optical images is 2.3 arcmin.

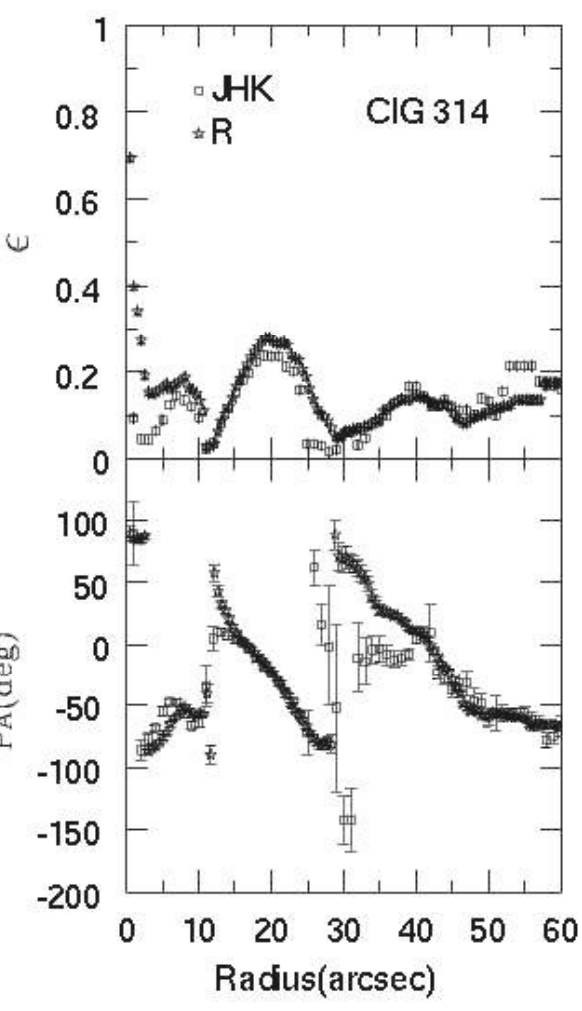


H. M. Hernández-Toledo and S. Ortega-Esbrí: Broad-band BVRI photometry of isolated spiral galaxies, Online Material p 11
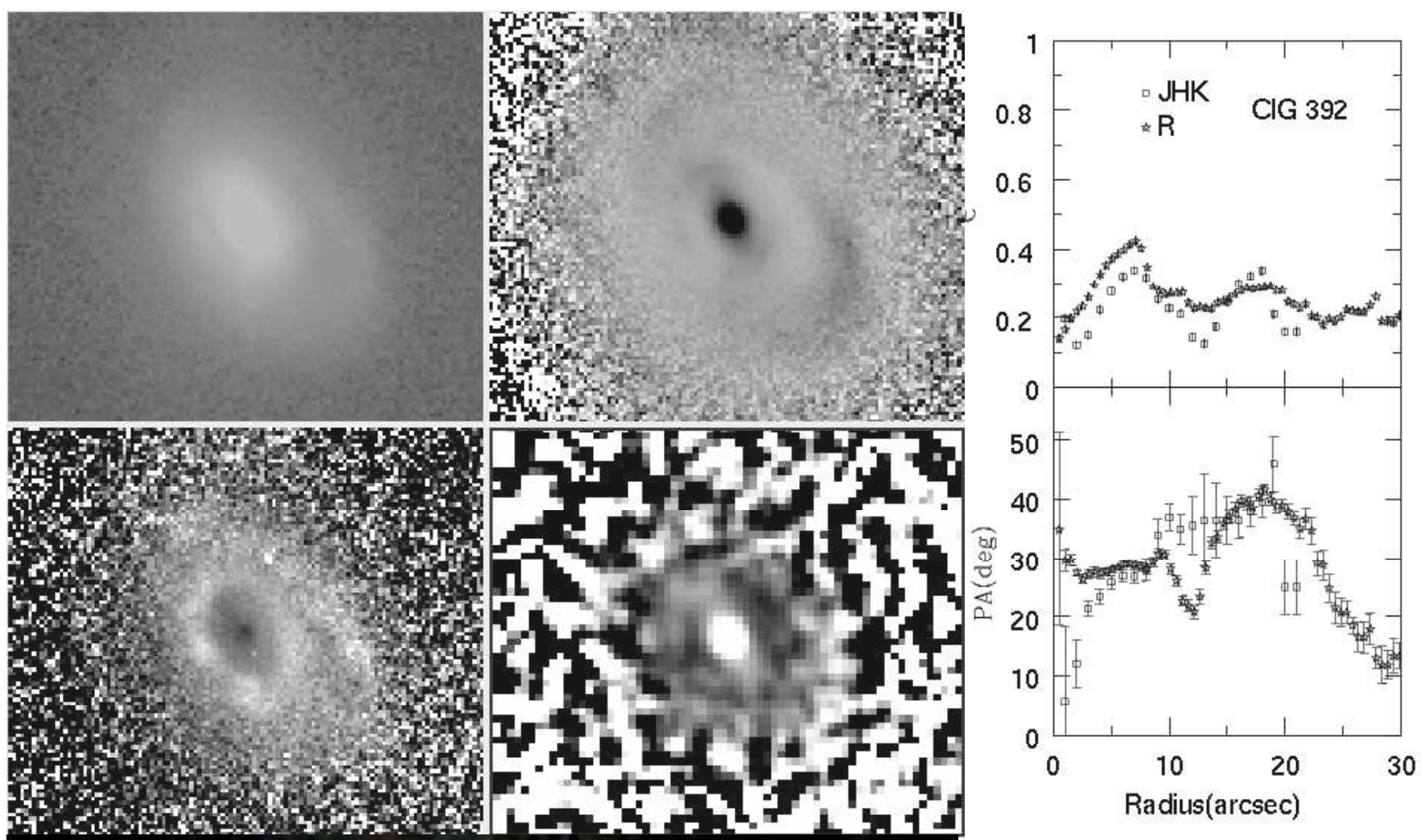

Fig. B.11. CIG 392 Mosaic. Same as Fig. 3. The major diameter of the galaxy in the optical images is 0.8 arcmin. 
H. M. Hernández-Toledo and S. Ortega-Esbrí: Broad-band BVRI photometry of isolated spiral galaxies, Online Material p 12
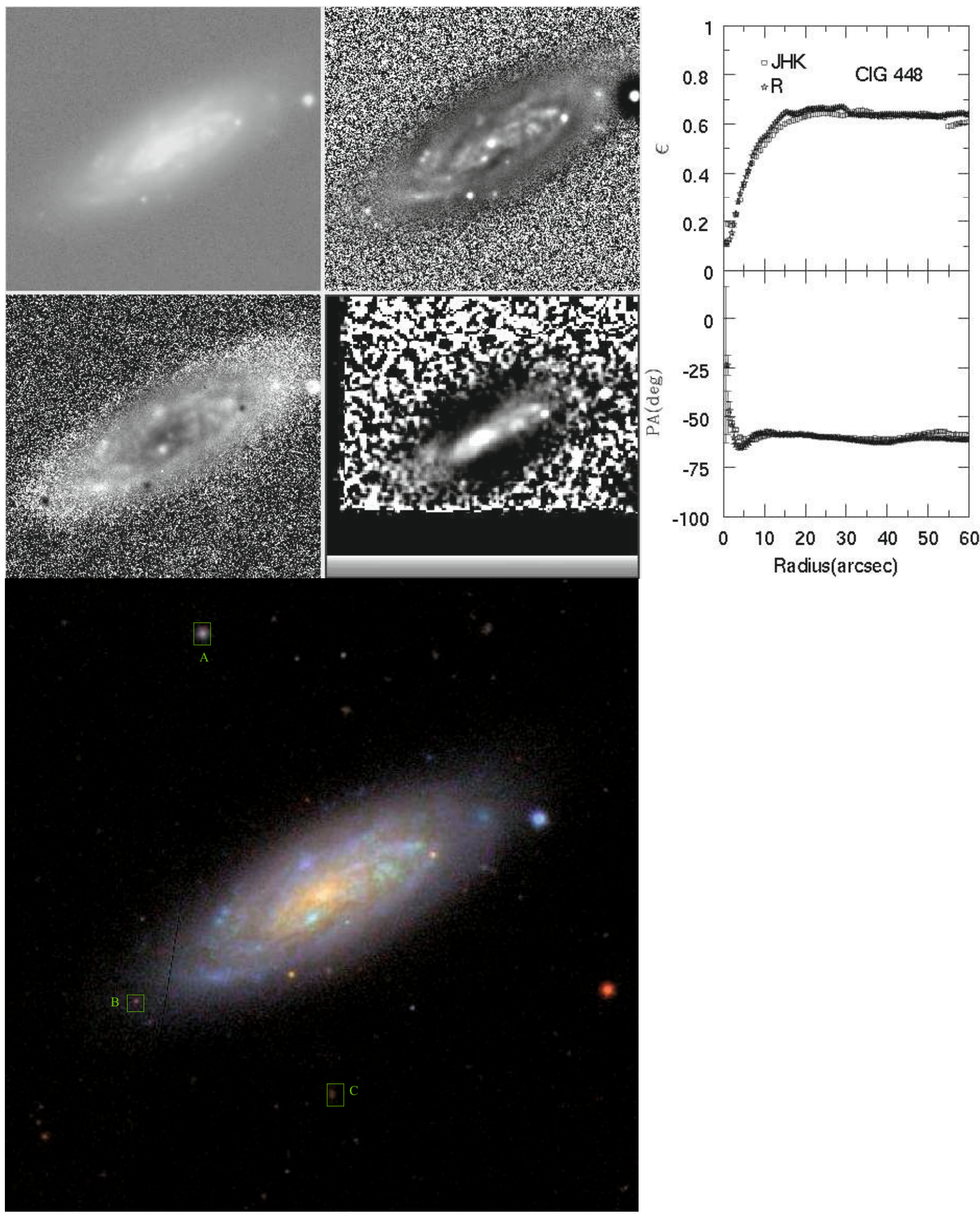

Fig. B.12. CIG 448 Mosaic. Same as Fig. 3. The major diameter of the galaxy in the optical images is 2.5 arcmin. 
H. M. Hernández-Toledo and S. Ortega-Esbrí: Broad-band BVRI photometry of isolated spiral galaxies, Online Material p 13
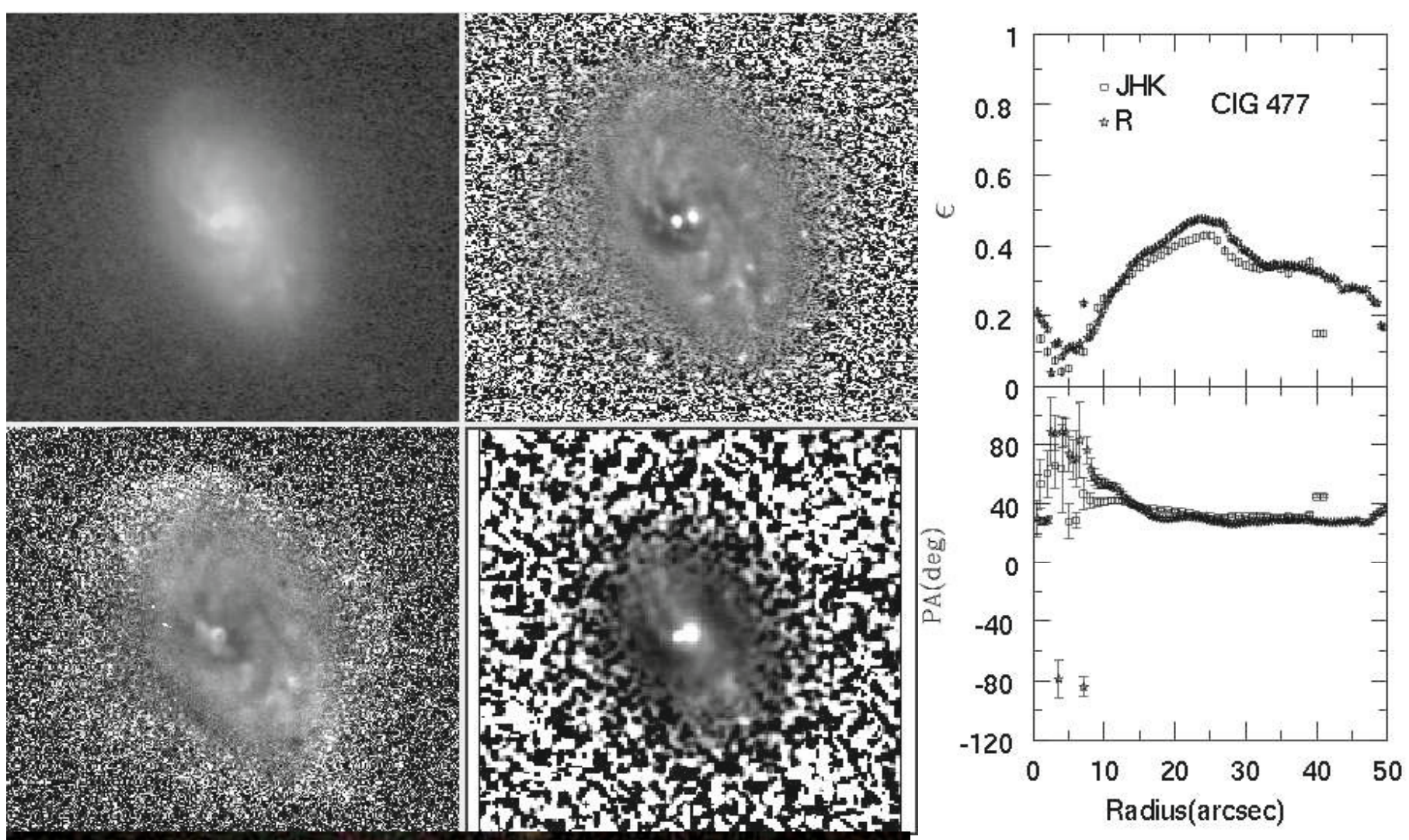

Fig. B.13. CIG 477 Mosaic. Same as Fig. 3. The major diameter of the galaxy in the optical images is 1.5 arcmin. 
H. M. Hernández-Toledo and S. Ortega-Esbrí: Broad-band BVRI photometry of isolated spiral galaxies, Online Material p 14
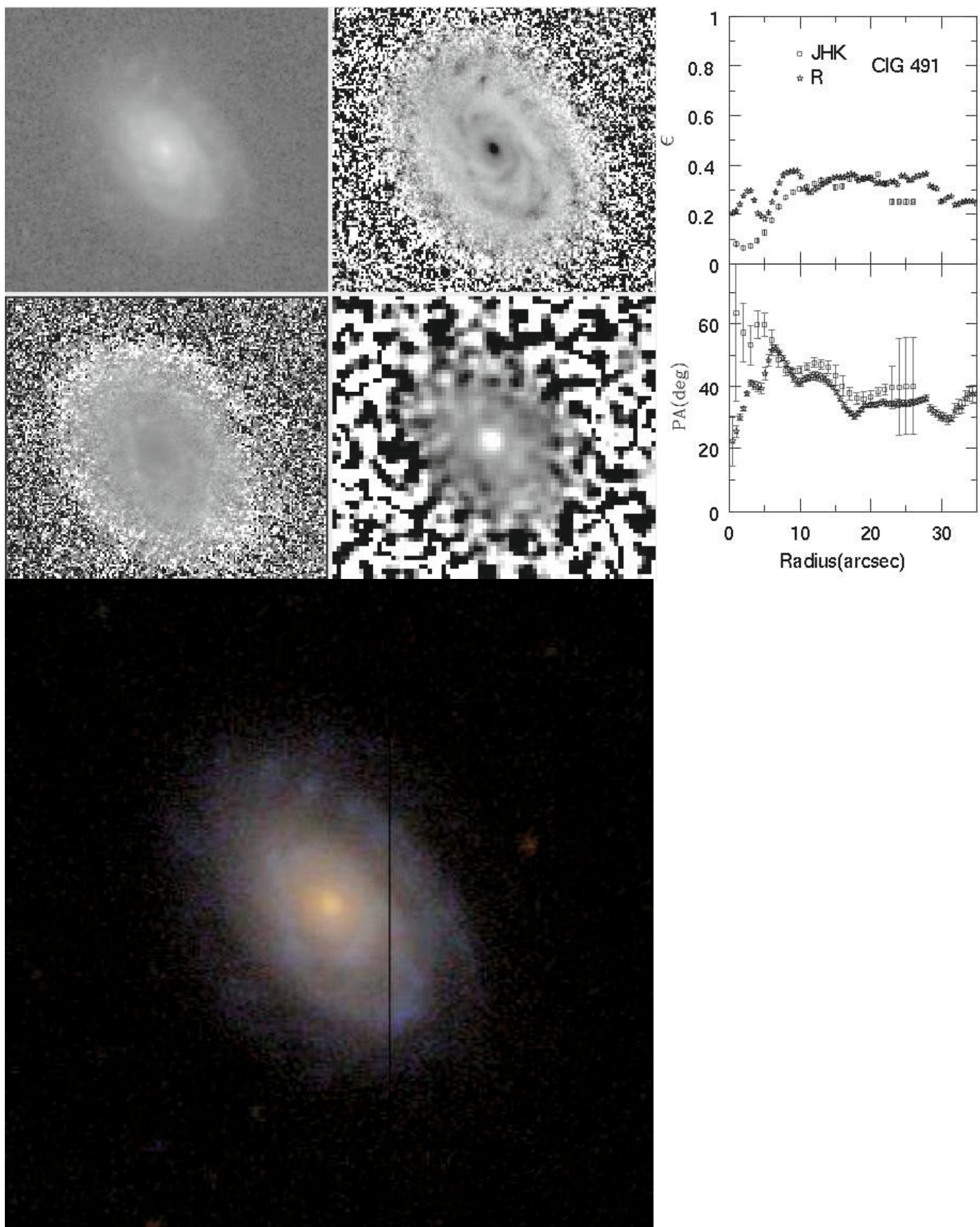

Fig. B.14. CIG 491 Mosaic. Same as Fig. 3. The major diameter of the galaxy in the optical images is $1.0 \operatorname{arcmin}$. 
H. M. Hernández-Toledo and S. Ortega-Esbrí: Broad-band BVRI photometry of isolated spiral galaxies, Online Material p 15
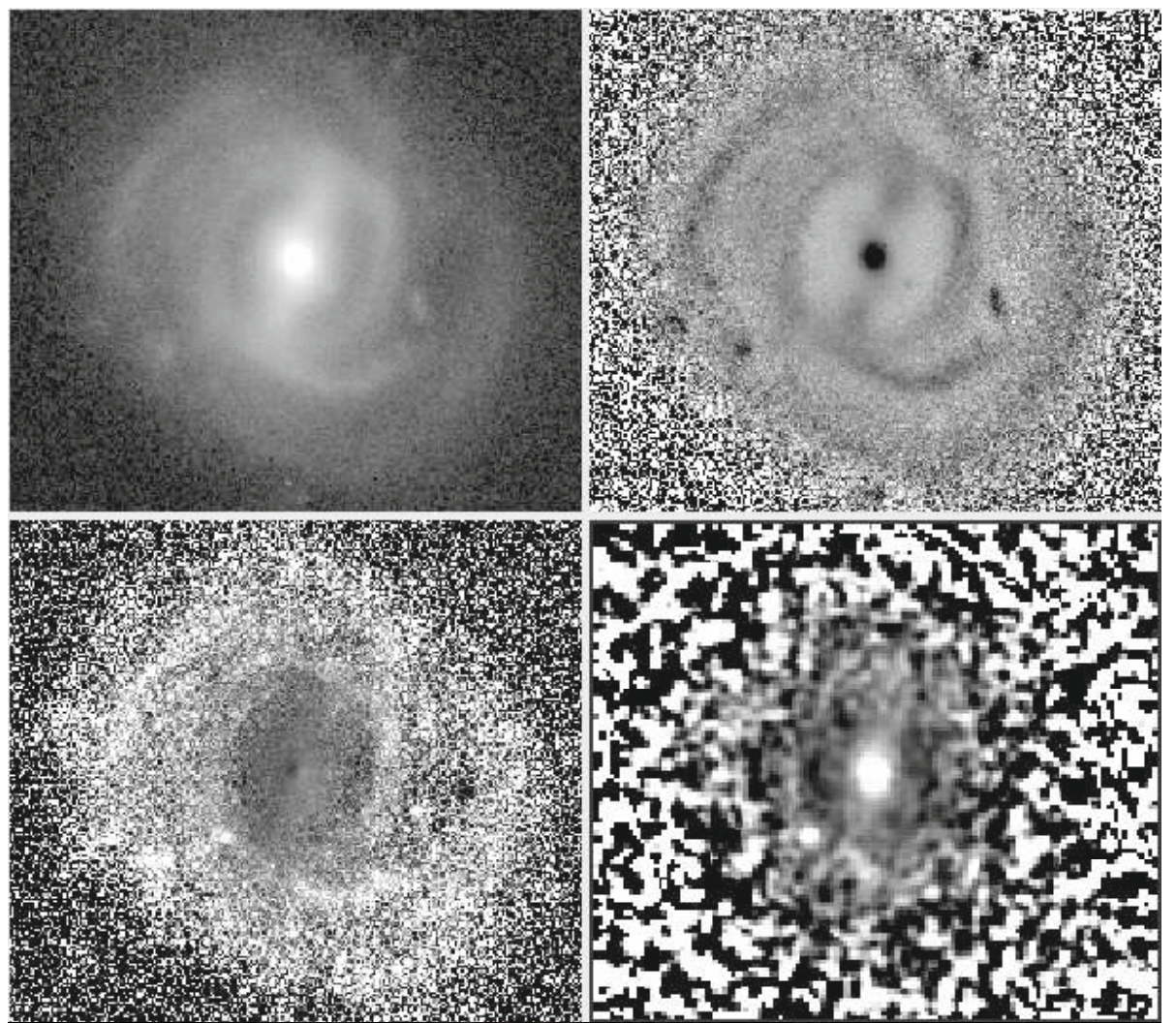

,
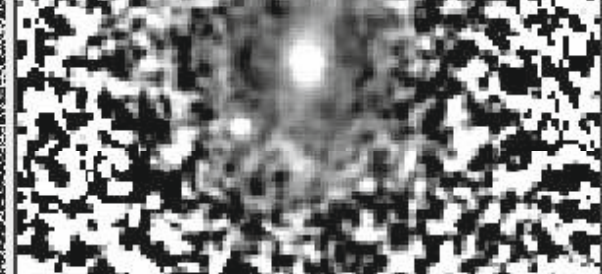

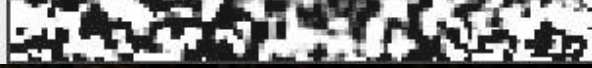

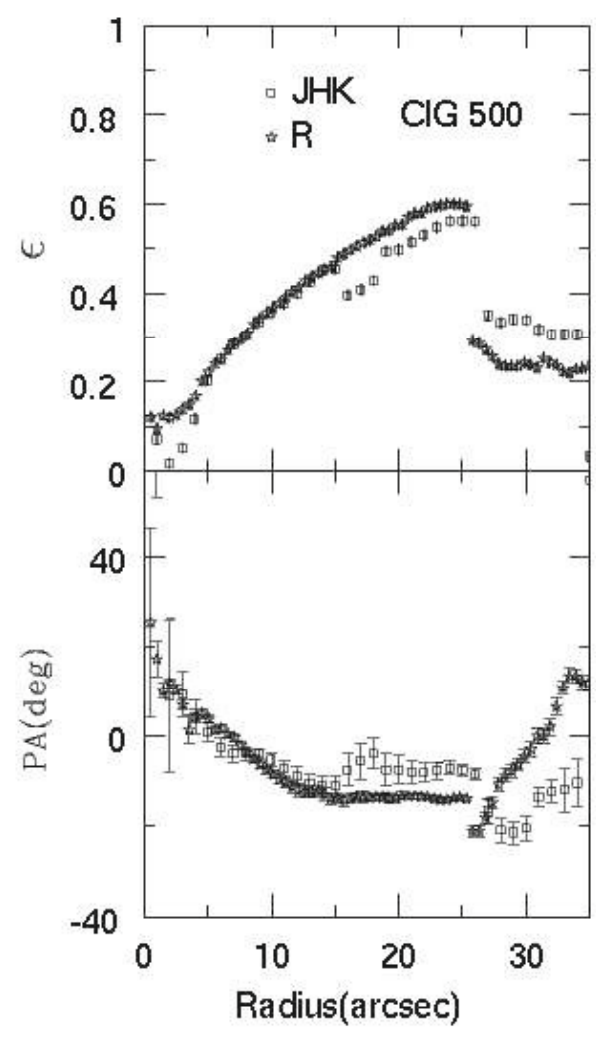

1.

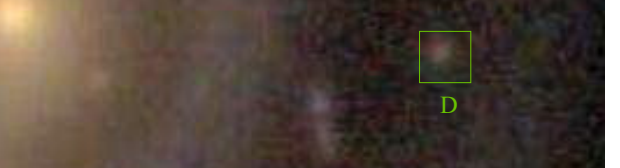

20.

B

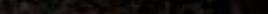

(1)

$-$

.

Fig. B.15. CIG 500 Mosaic. Same as Fig. 3. The major diameter of the galaxy in the optical images is 1.6 arcmin. 
H. M. Hernández-Toledo and S. Ortega-Esbrí: Broad-band BVRI photometry of isolated spiral galaxies, Online Material p 16
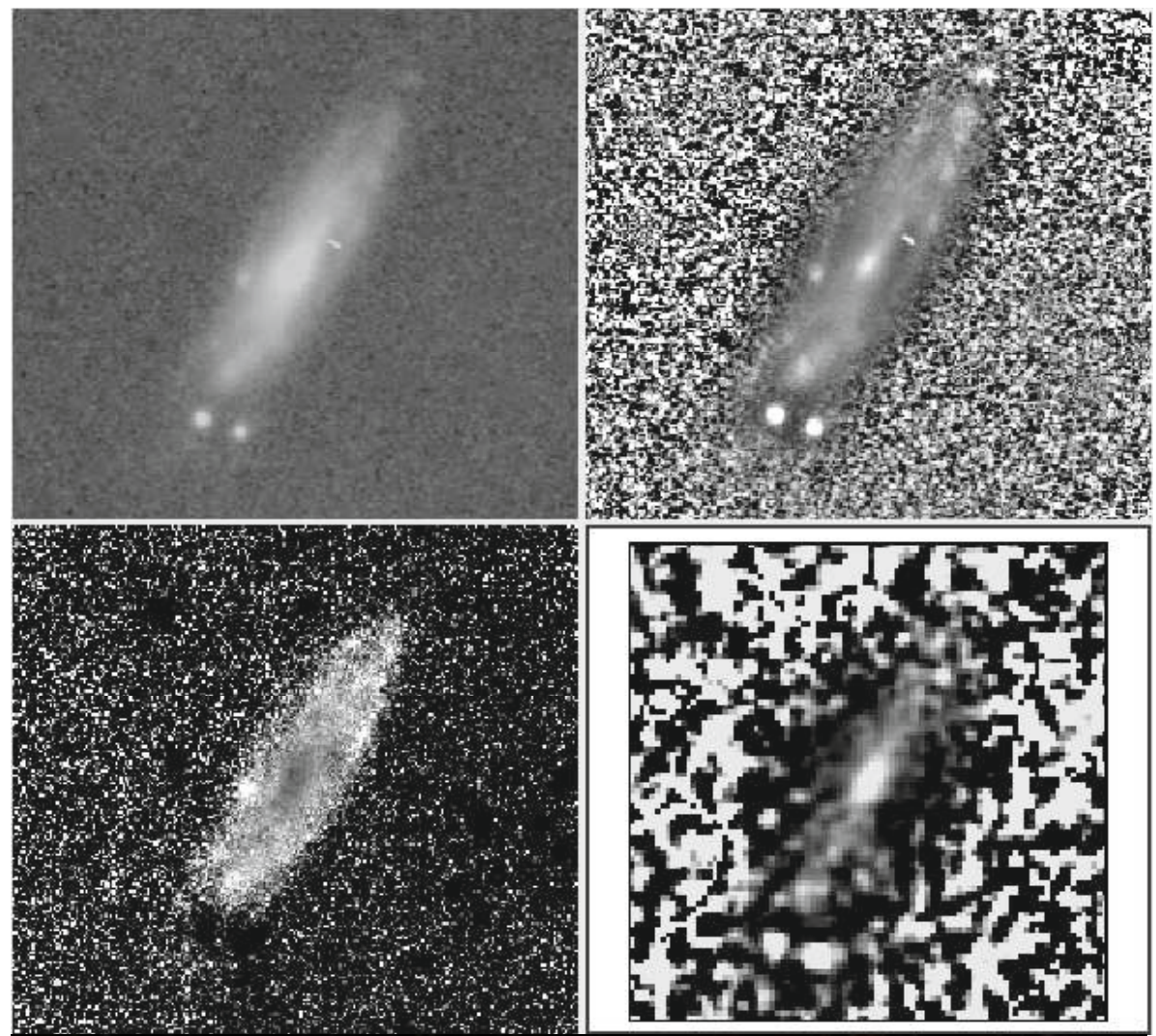
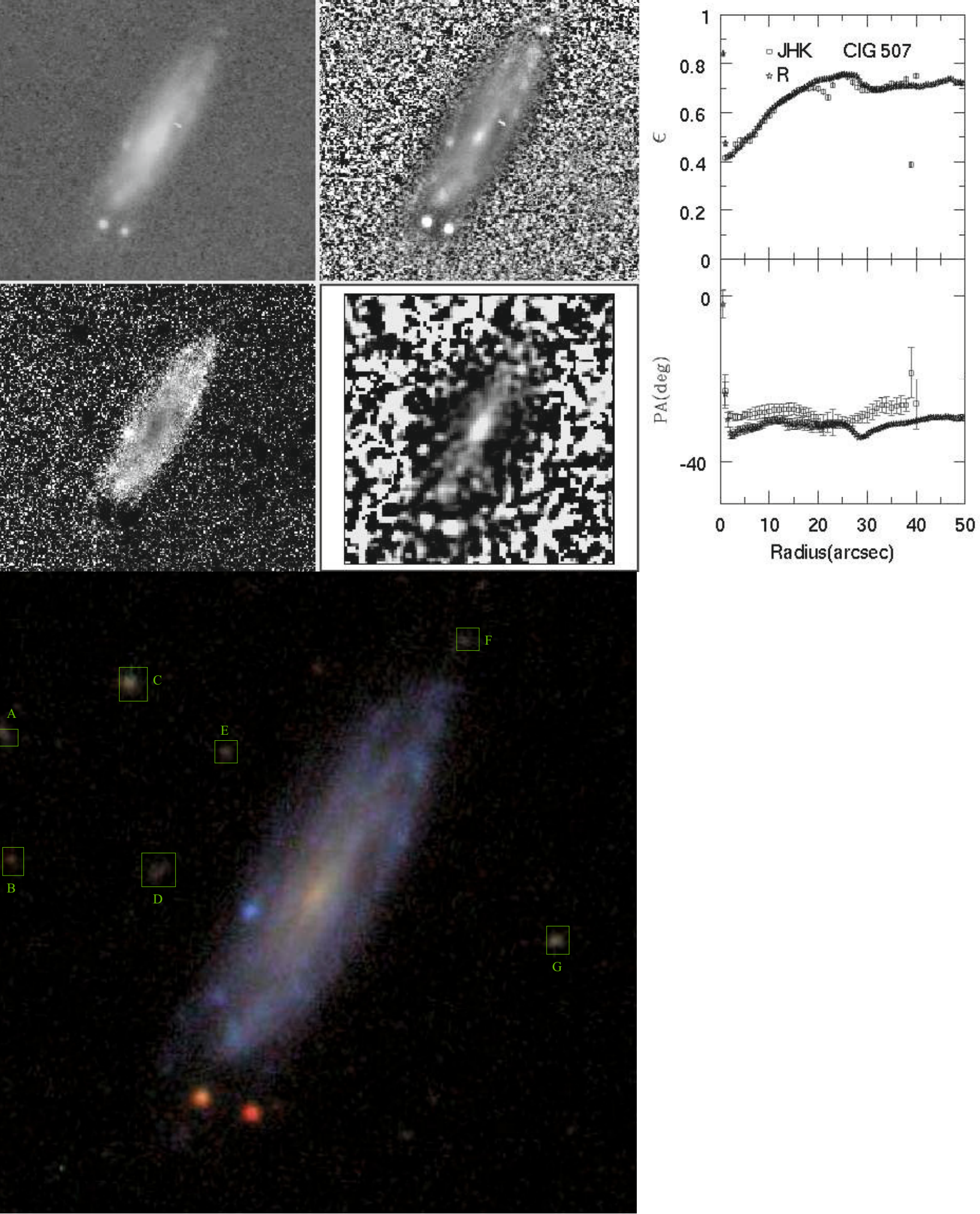

Fig. B.16. CIG 507 Mosaic. Same as Fig. 3. The major diameter of the galaxy in the optical images is 1.5 arcmin. 
H. M. Hernández-Toledo and S. Ortega-Esbrí: Broad-band BVRI photometry of isolated spiral galaxies, Online Material p 17
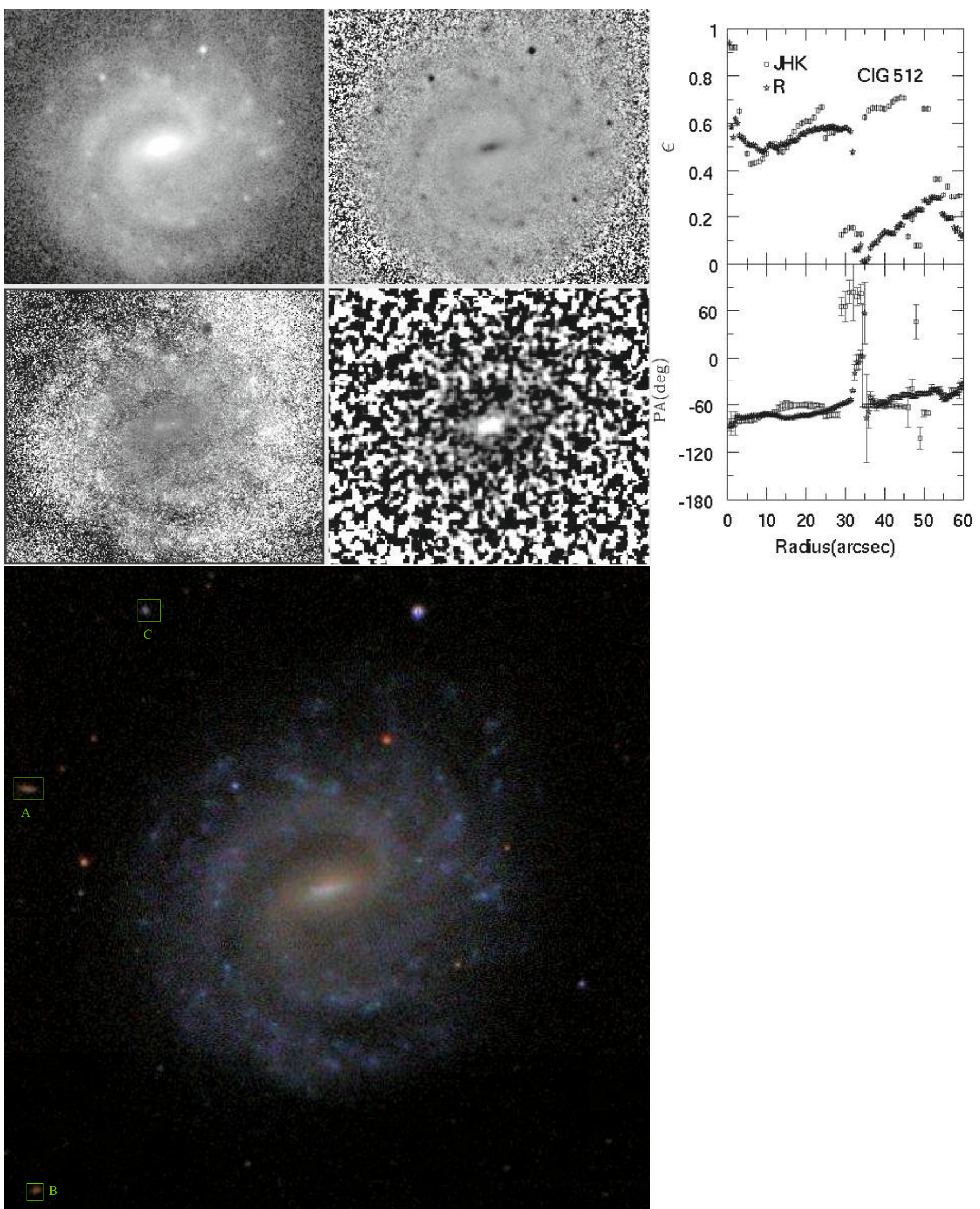

Fig. B.17. CIG 512 Mosaic. Same as Fig. 3. The major diameter of the galaxy in the optical images is 1.6 arcmin. 
H. M. Hernández-Toledo and S. Ortega-Esbrí: Broad-band BVRI photometry of isolated spiral galaxies, Online Material p 18
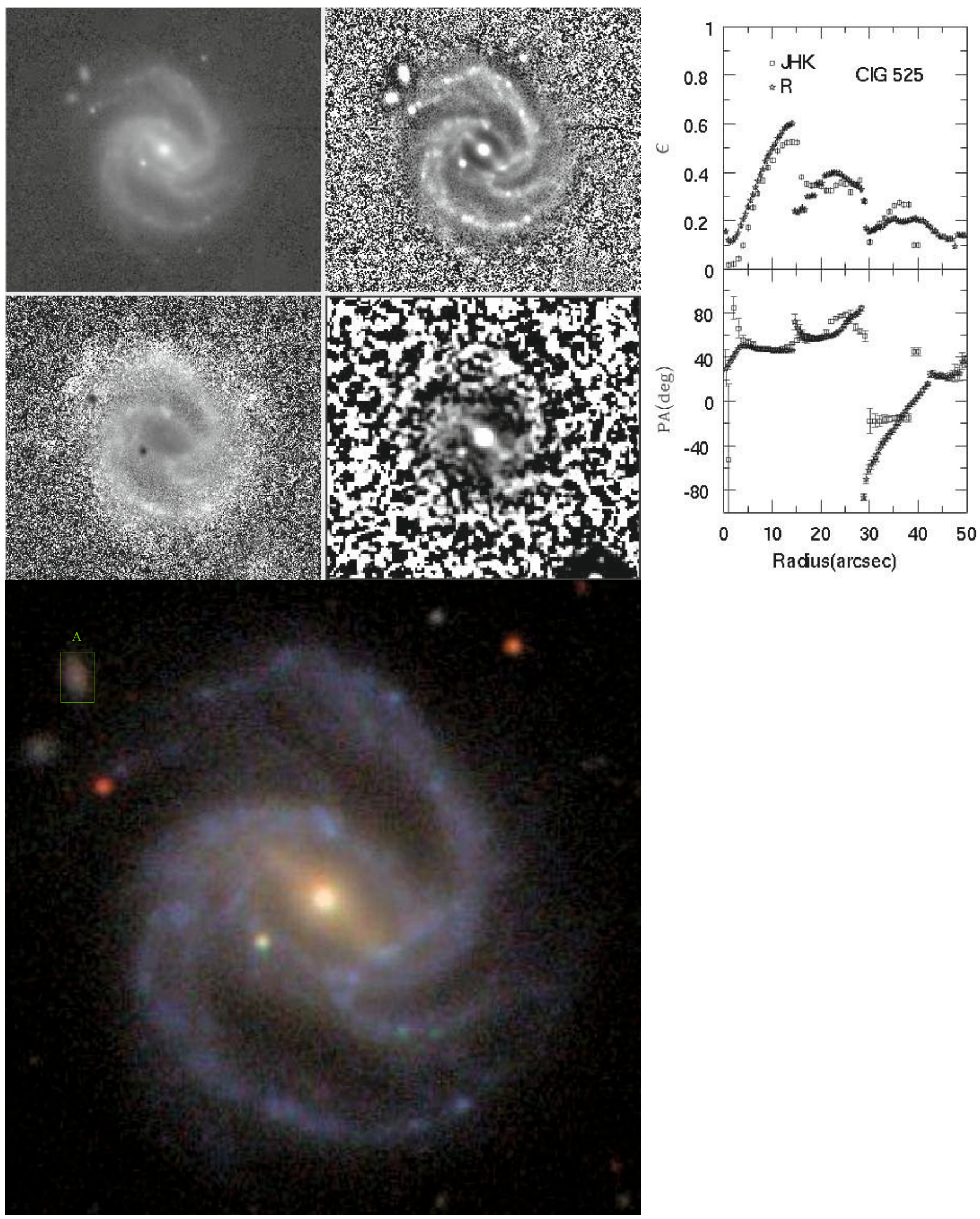

Fig. B.18. CIG 525 Mosaic. Same as Fig. 3. The major diameter of the galaxy in the optical images is 1.4 arcmin. 
H. M. Hernández-Toledo and S. Ortega-Esbrí: Broad-band BVRI photometry of isolated spiral galaxies, Online Material p 19
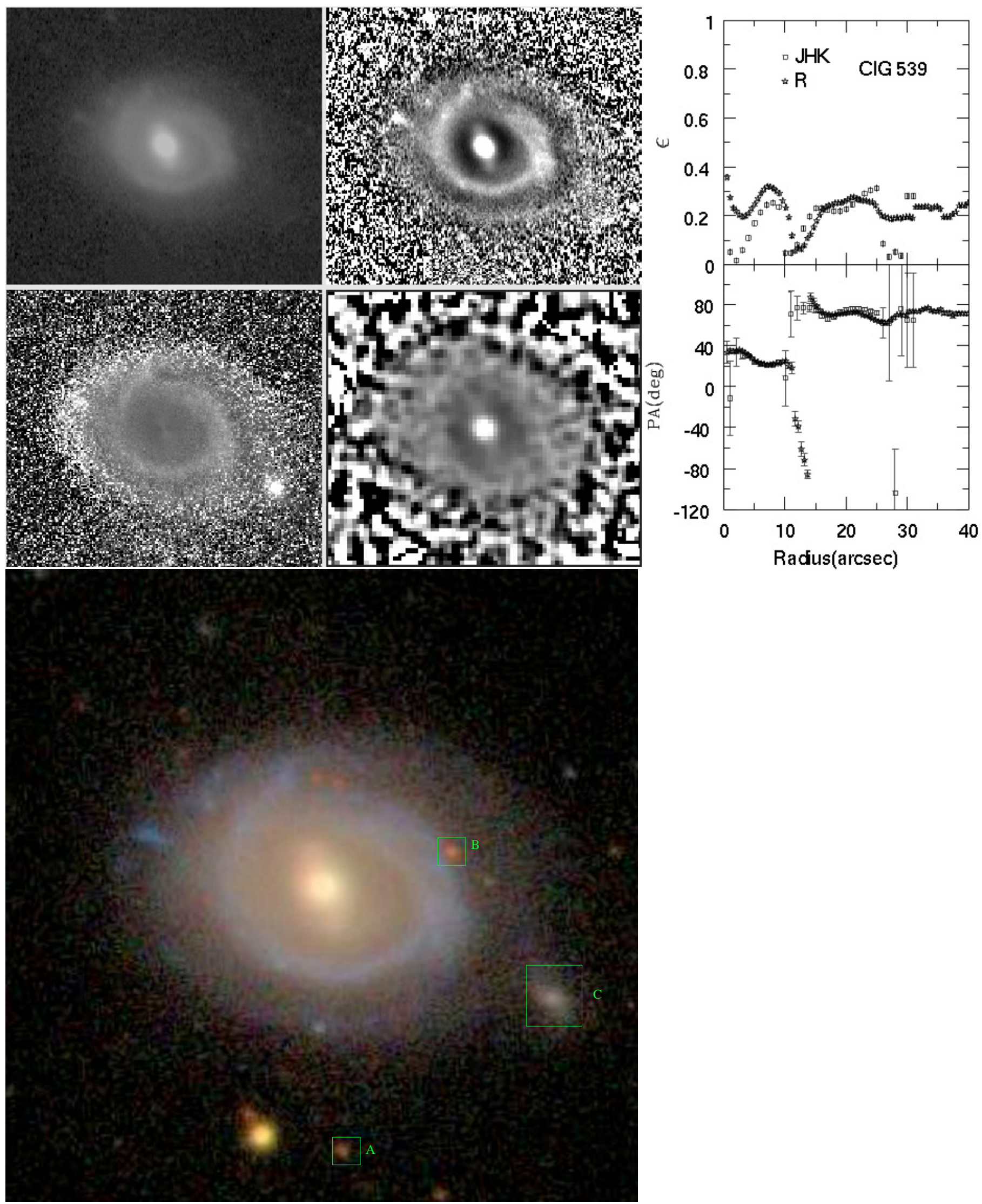

Fig. B.19. CIG 539 Mosaic. Same as Fig. 3. The major diameter of the galaxy in the optical images is 1.1 arcmin. 
H. M. Hernández-Toledo and S. Ortega-Esbrí: Broad-band BVRI photometry of isolated spiral galaxies, Online Material p 20
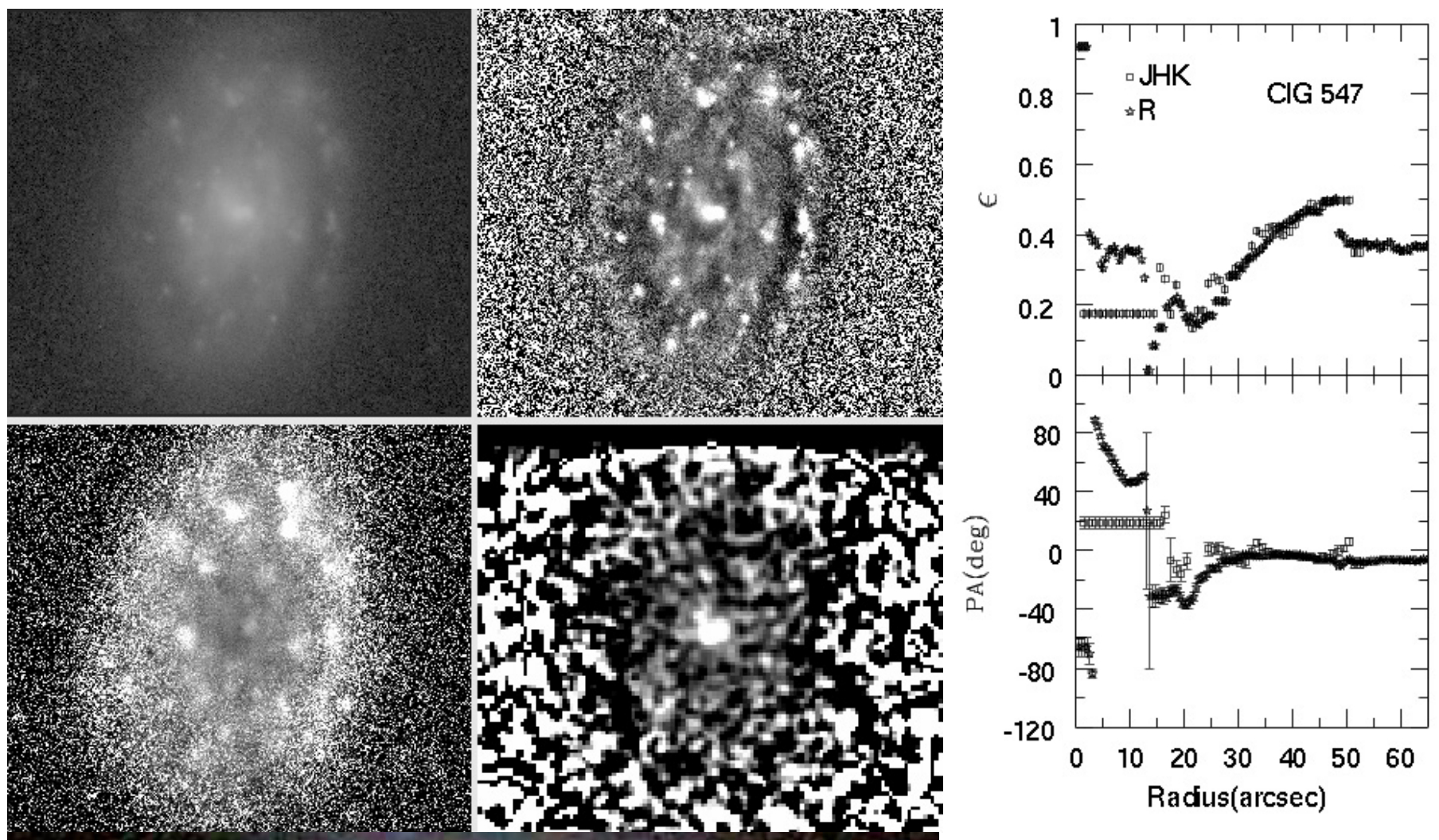

Radus(arcsec)

Fig. B.20. CIG 547 Mosaic. Same as Fig. 3. The major diameter of the galaxy in the optical images is 2.0 arcmin. 
H. M. Hernández-Toledo and S. Ortega-Esbrí: Broad-band BVRI photometry of isolated spiral galaxies, Online Material p 21
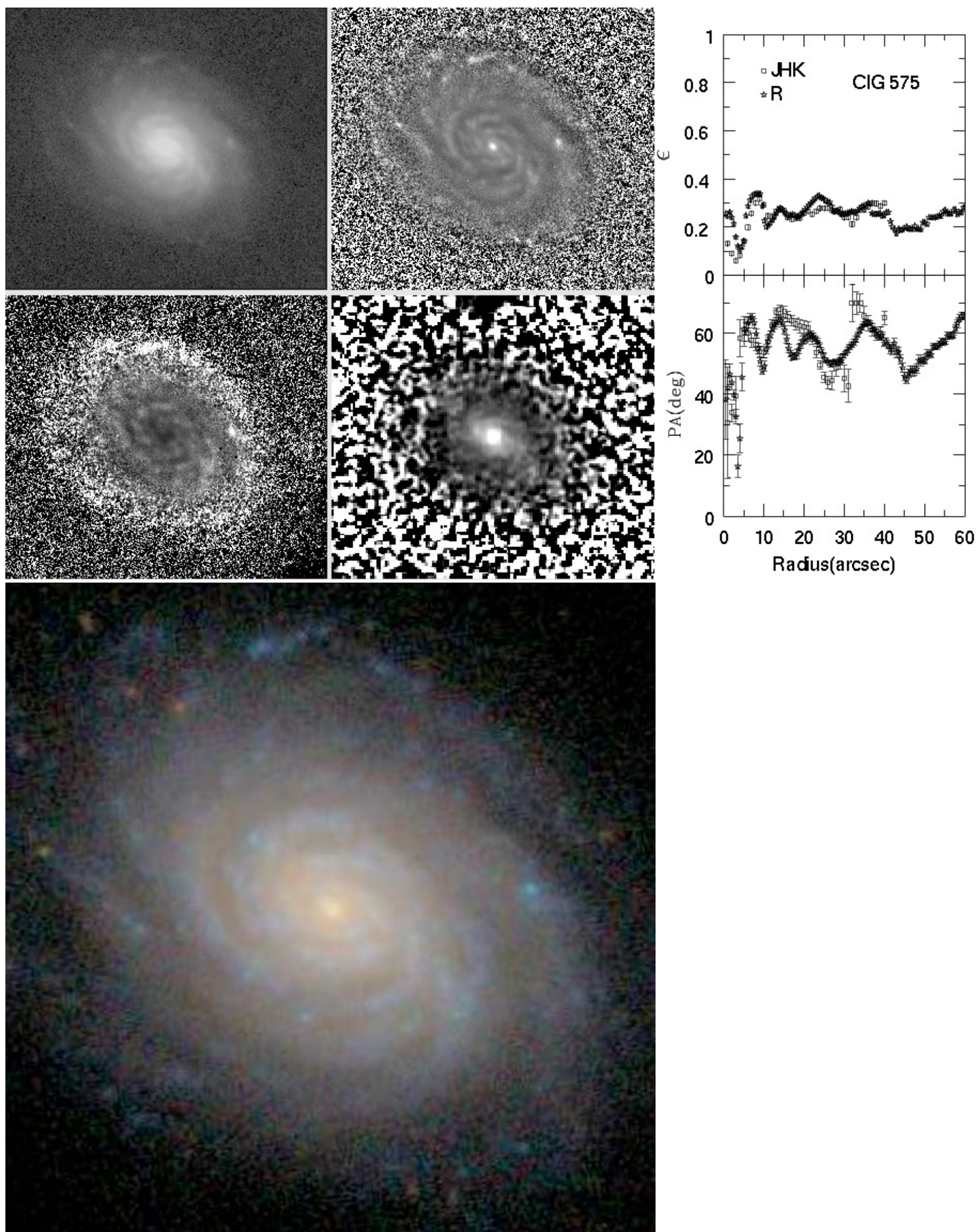

Fig. B.21. CIG 575 Mosaic. Same as Fig. 3. The major diameter of the galaxy in the optical images is 1.7 arcmin. 
H. M. Hernández-Toledo and S. Ortega-Esbrí: Broad-band BVRI photometry of isolated spiral galaxies, Online Material p 22
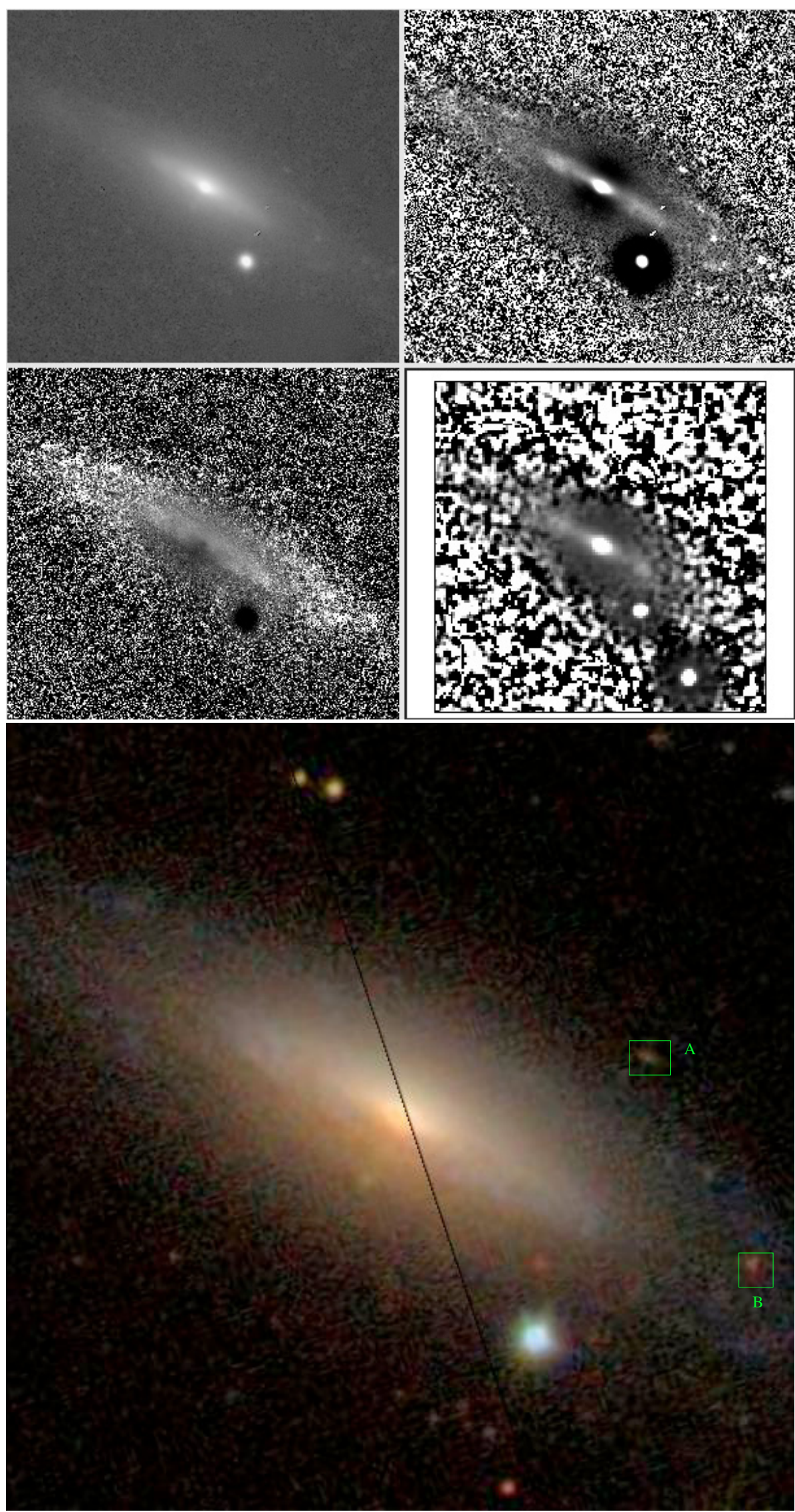

Fig. B.22. CIG 616 Mosaic. Same as Fig. 3. The major diameter of the galaxy in the optical images is 2.6 arcmin.

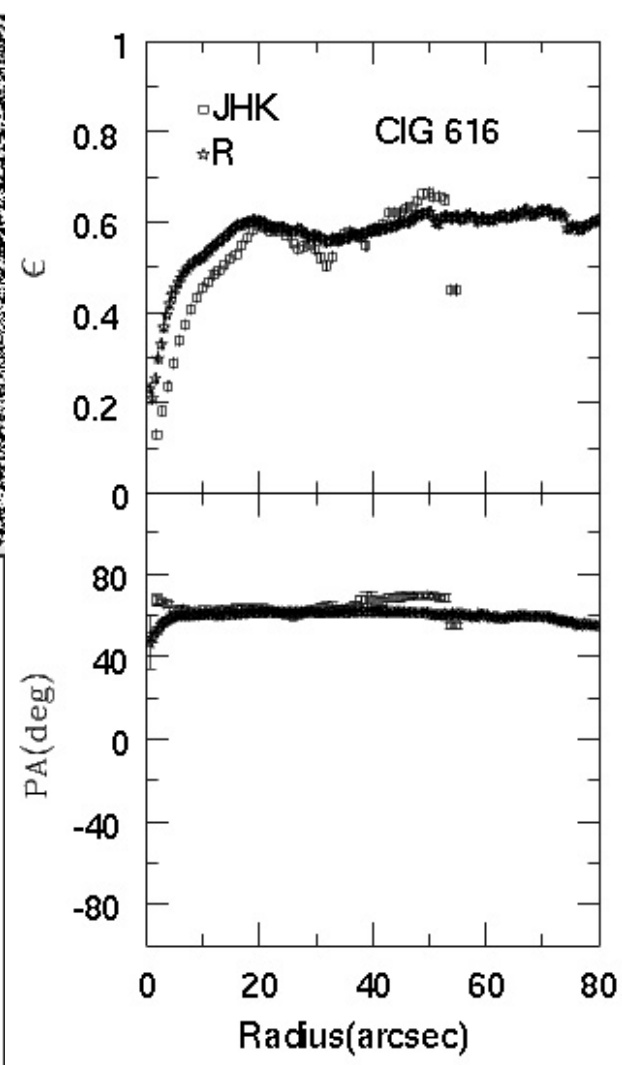


H. M. Hernández-Toledo and S. Ortega-Esbrí: Broad-band BVRI photometry of isolated spiral galaxies, Online Material p 23
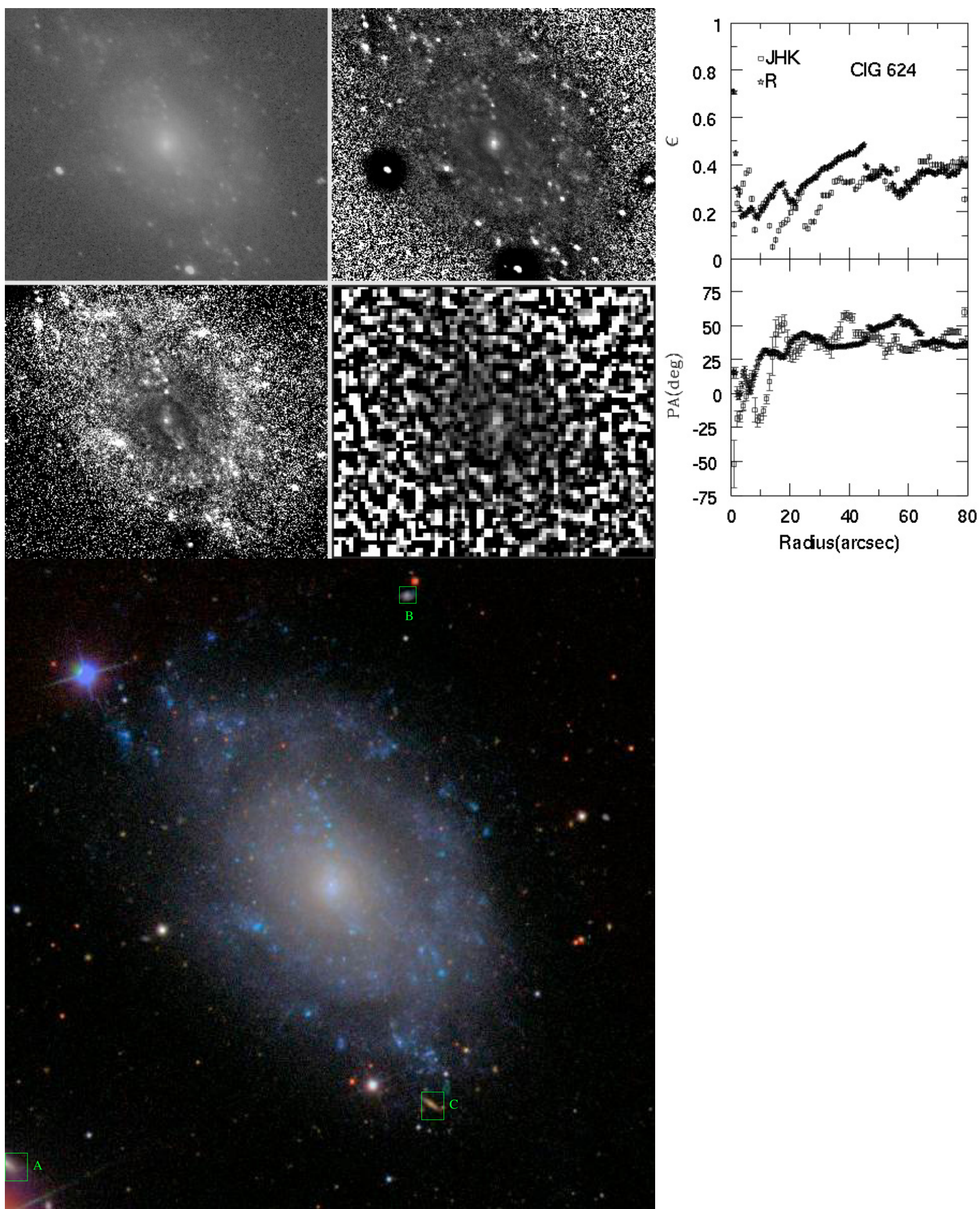

Fig. B.23. CIG 624 Mosaic. Same as Fig. 3. The major diameter of the galaxy in the optical images is 5.8 arcmin. 
H. M. Hernández-Toledo and S. Ortega-Esbrí: Broad-band BVRI photometry of isolated spiral galaxies, Online Material p 24
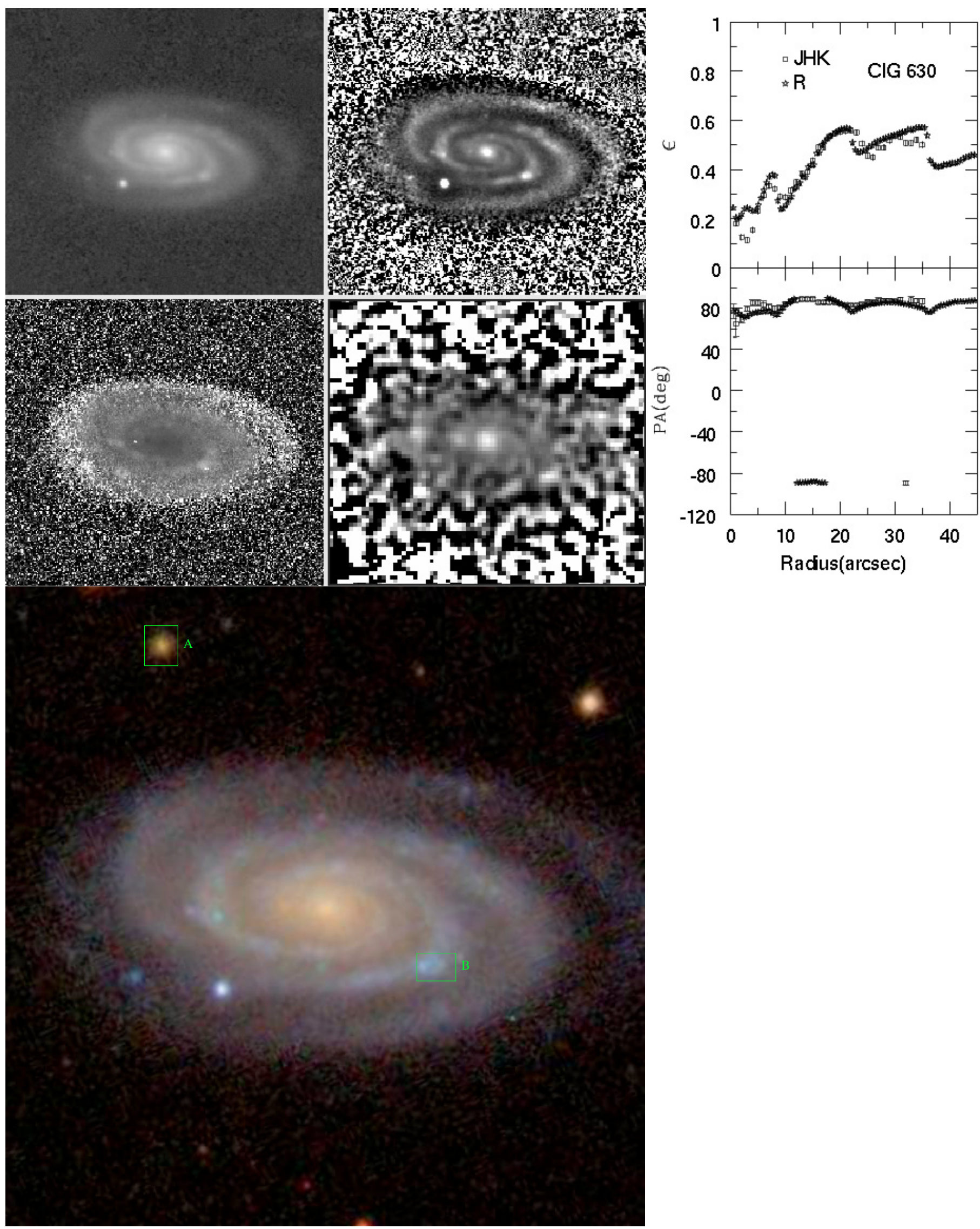

Fig. B.24. CIG 630 Mosaic. Same as Fig. 3. The major diameter of the galaxy in the optical images is 1.6 arcmin. 
H. M. Hernández-Toledo and S. Ortega-Esbrí: Broad-band BVRI photometry of isolated spiral galaxies, Online Material p 25
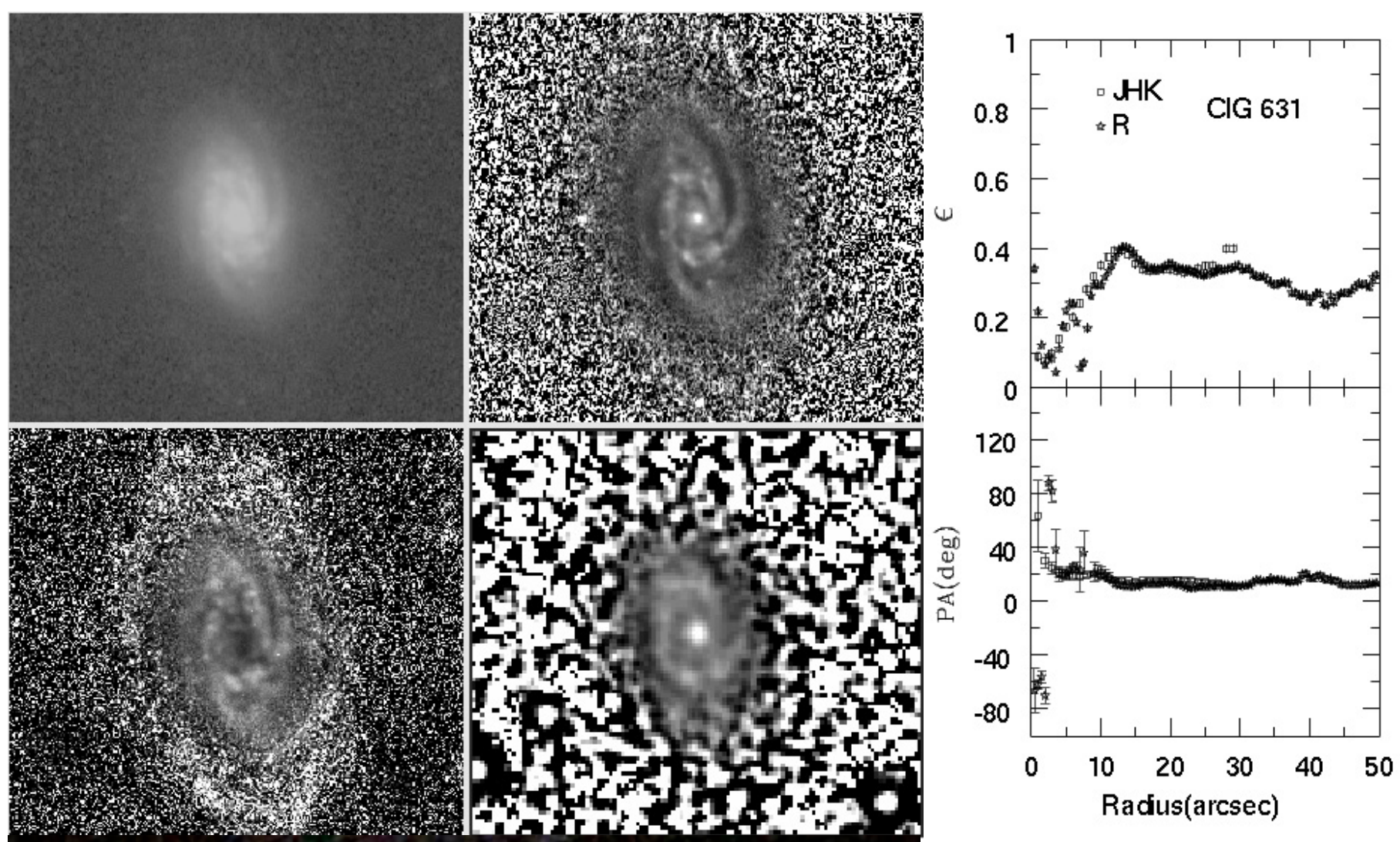

Fig. B.25. CIG 631 Mosaic. Same as Fig. 3. The major diameter of the galaxy in the optical images is 1.6 arcmin. 
H. M. Hernández-Toledo and S. Ortega-Esbrí: Broad-band BVRI photometry of isolated spiral galaxies, Online Material p 26
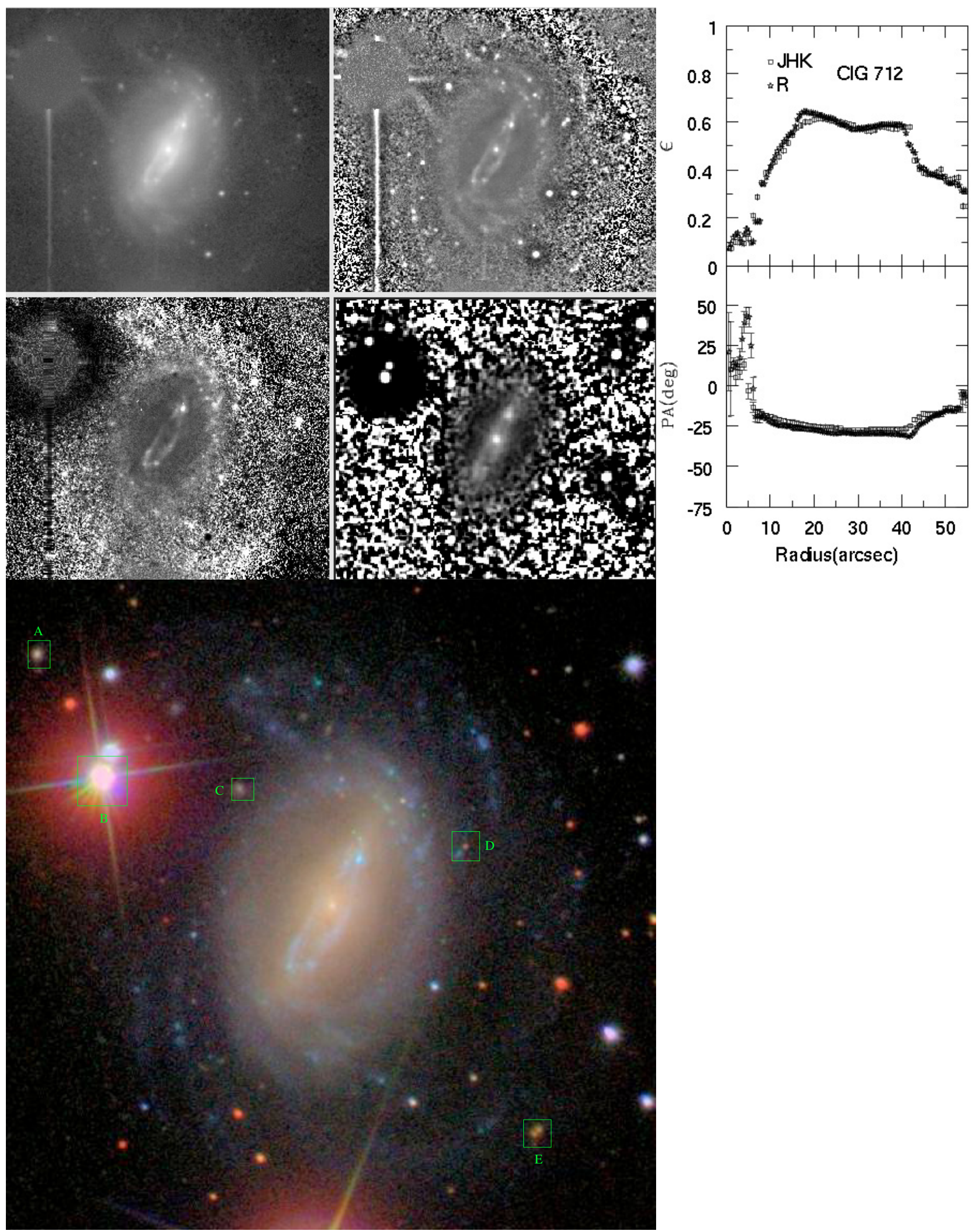

Fig. B.26. CIG 712 Mosaic. Same as Fig. 3. The major diameter of the galaxy in the optical images is 2.1 arcmin. 
H. M. Hernández-Toledo and S. Ortega-Esbrí: Broad-band BVRI photometry of isolated spiral galaxies, Online Material p 27
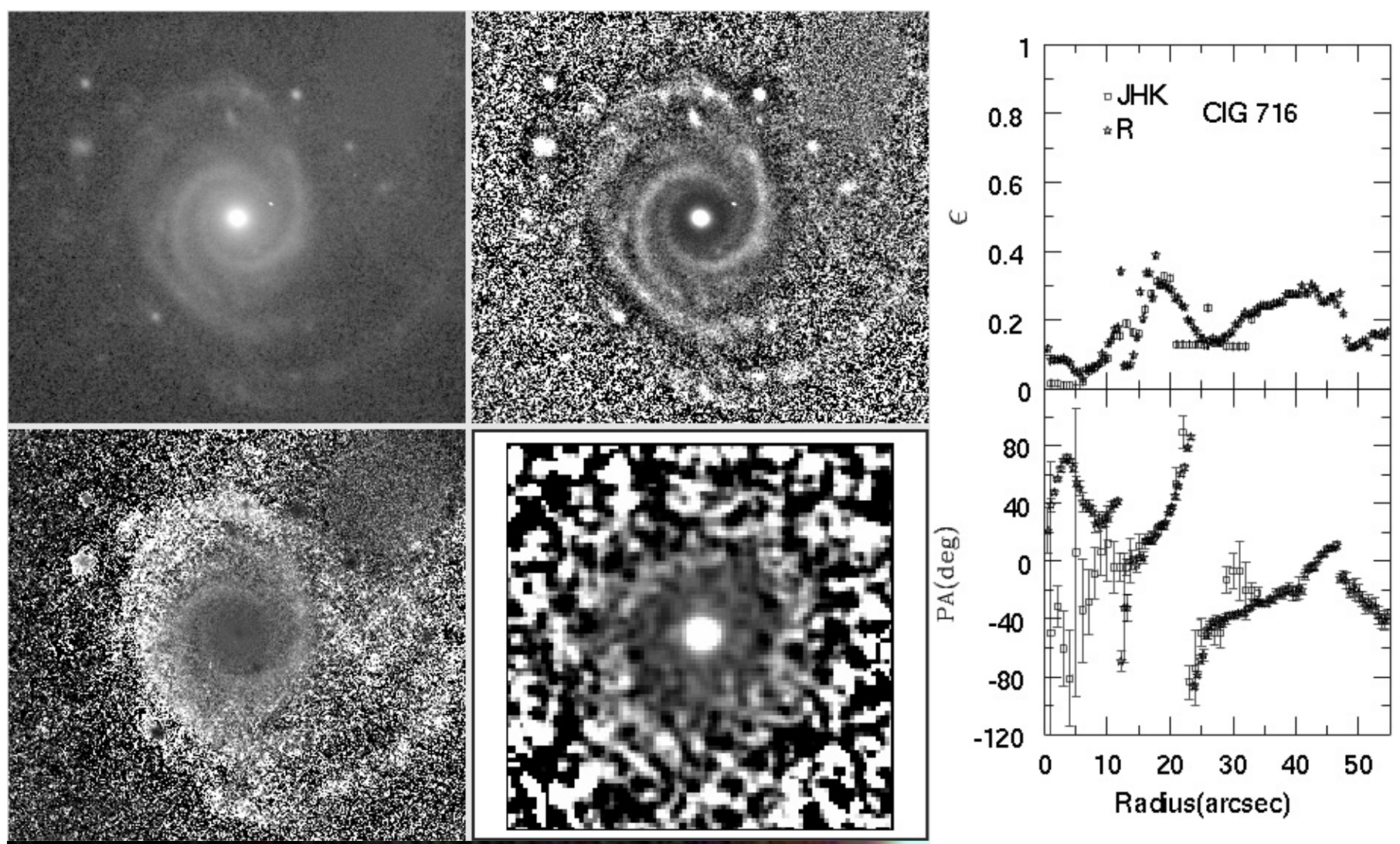

Fig. B.27. CIG 716 Mosaic. Same as Fig. 3. The major diameter of the galaxy in the optical images is 2.7 arcmin. 
H. M. Hernández-Toledo and S. Ortega-Esbrí: Broad-band BVRI photometry of isolated spiral galaxies, Online Material p 28
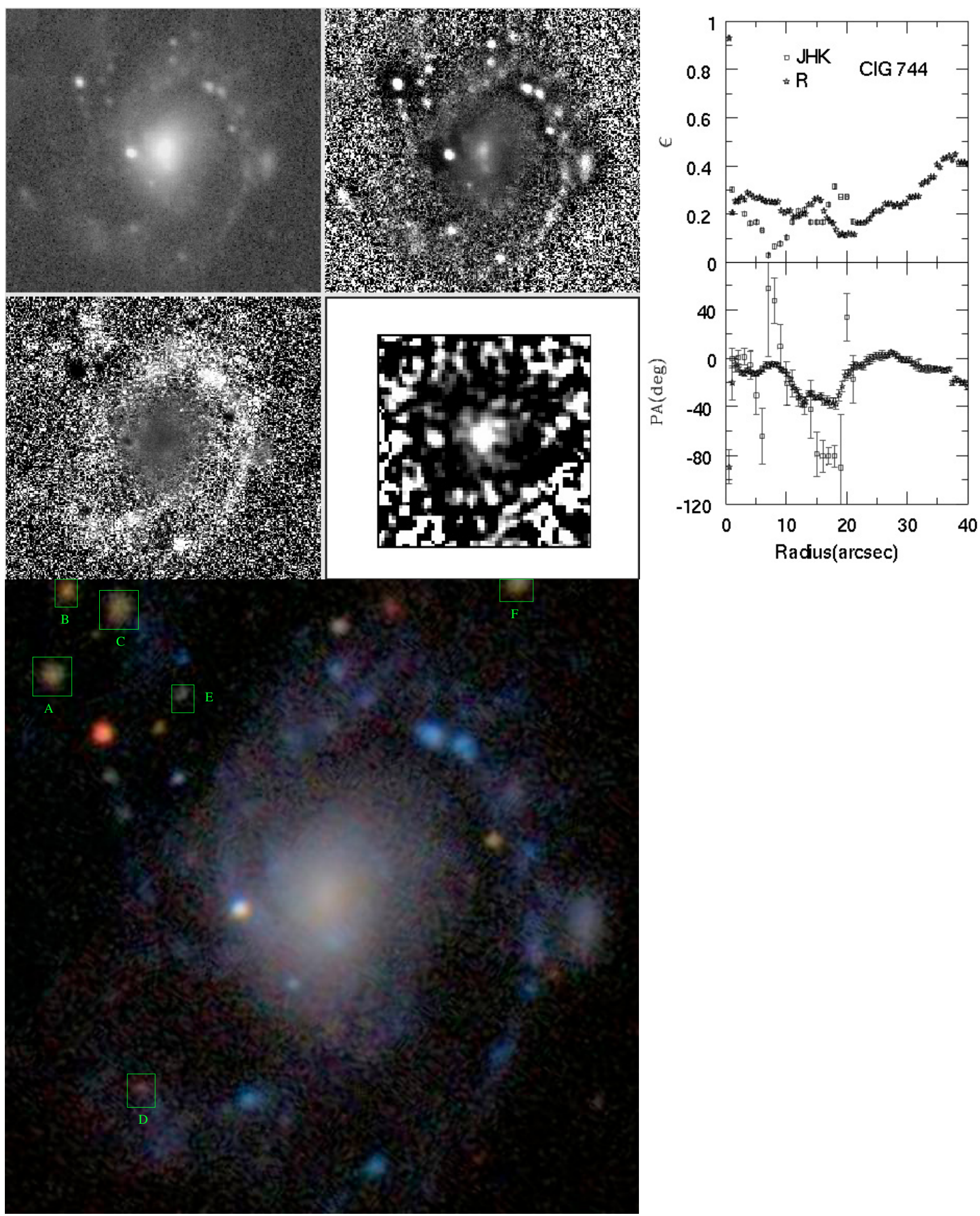

Fig. B.28. CIG 744 Mosaic. Same as Fig. 3. The major diameter of the galaxy in the optical images is 0.8 arcmin. 
H. M. Hernández-Toledo and S. Ortega-Esbrí: Broad-band BVRI photometry of isolated spiral galaxies, Online Material p 29
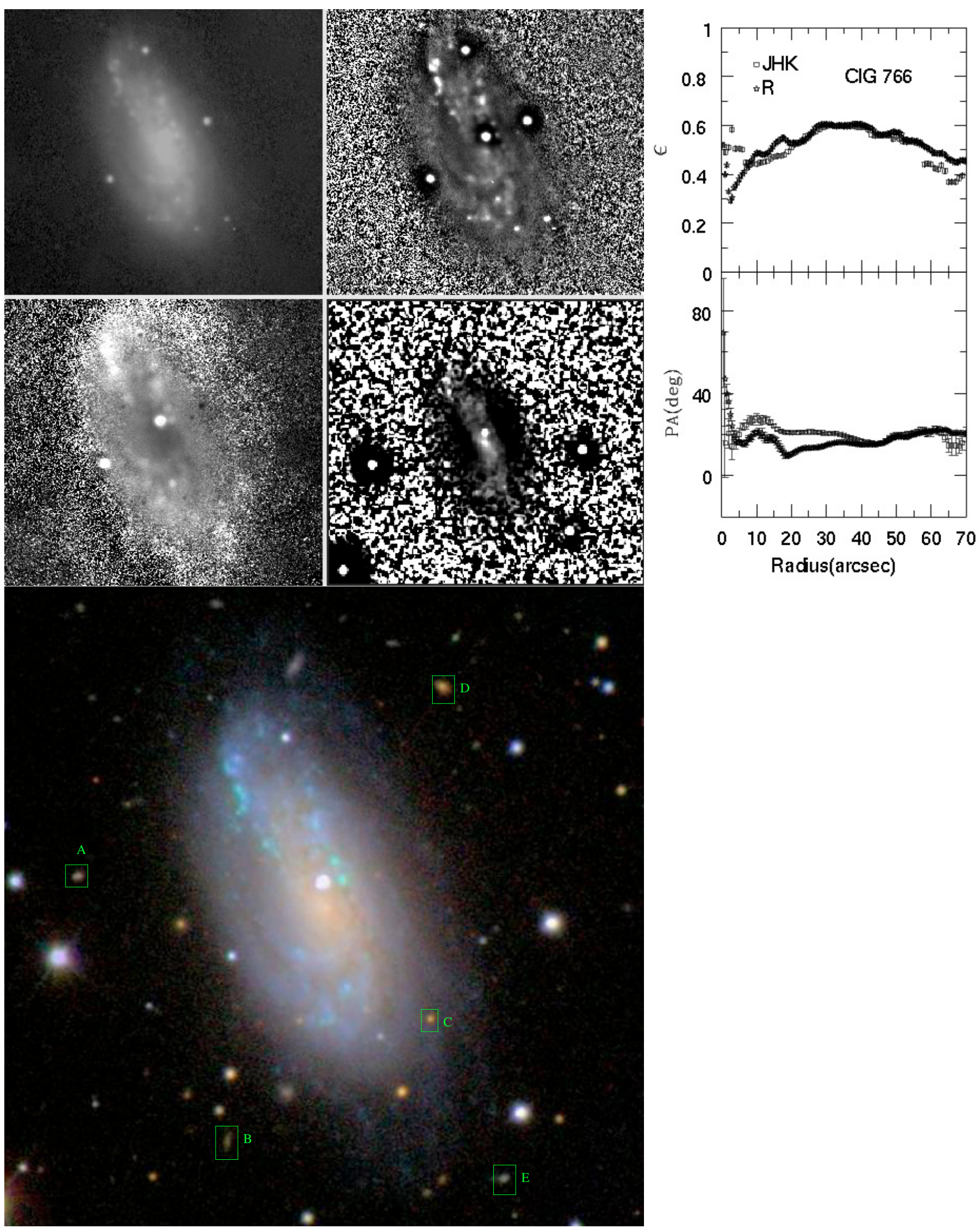

Fig. B.29. CIG 766 Mosaic. Same as Fig. 3. The major diameter of the galaxy in the optical images is 3.0 arcmin. 
H. M. Hernández-Toledo and S. Ortega-Esbrí: Broad-band BVRI photometry of isolated spiral galaxies, Online Material p 30
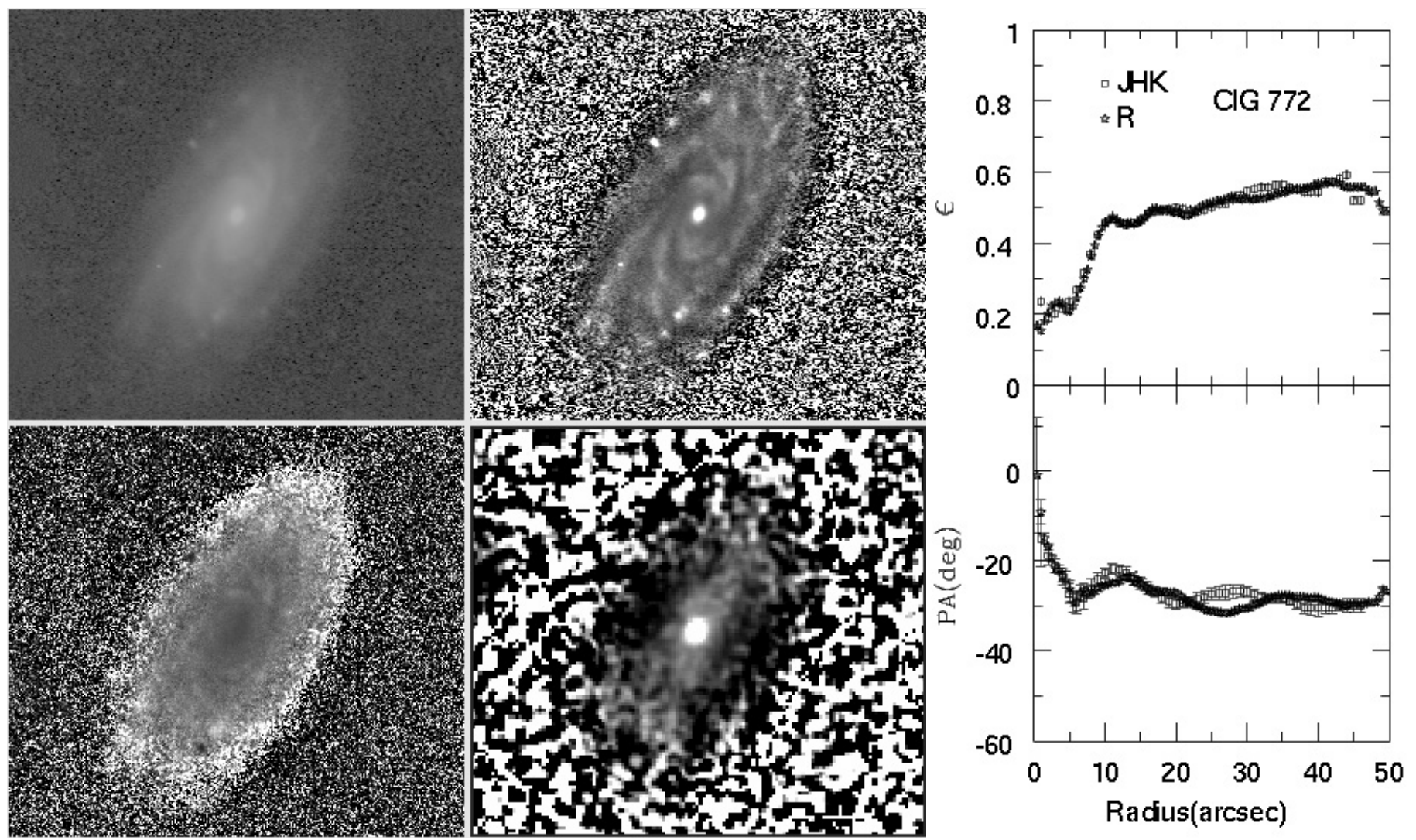

Fig. B.30. CIG 772 Mosaic. Same as Fig. 3. The major diameter of the galaxy in the optical images is $2.2 \operatorname{arcmin}$.
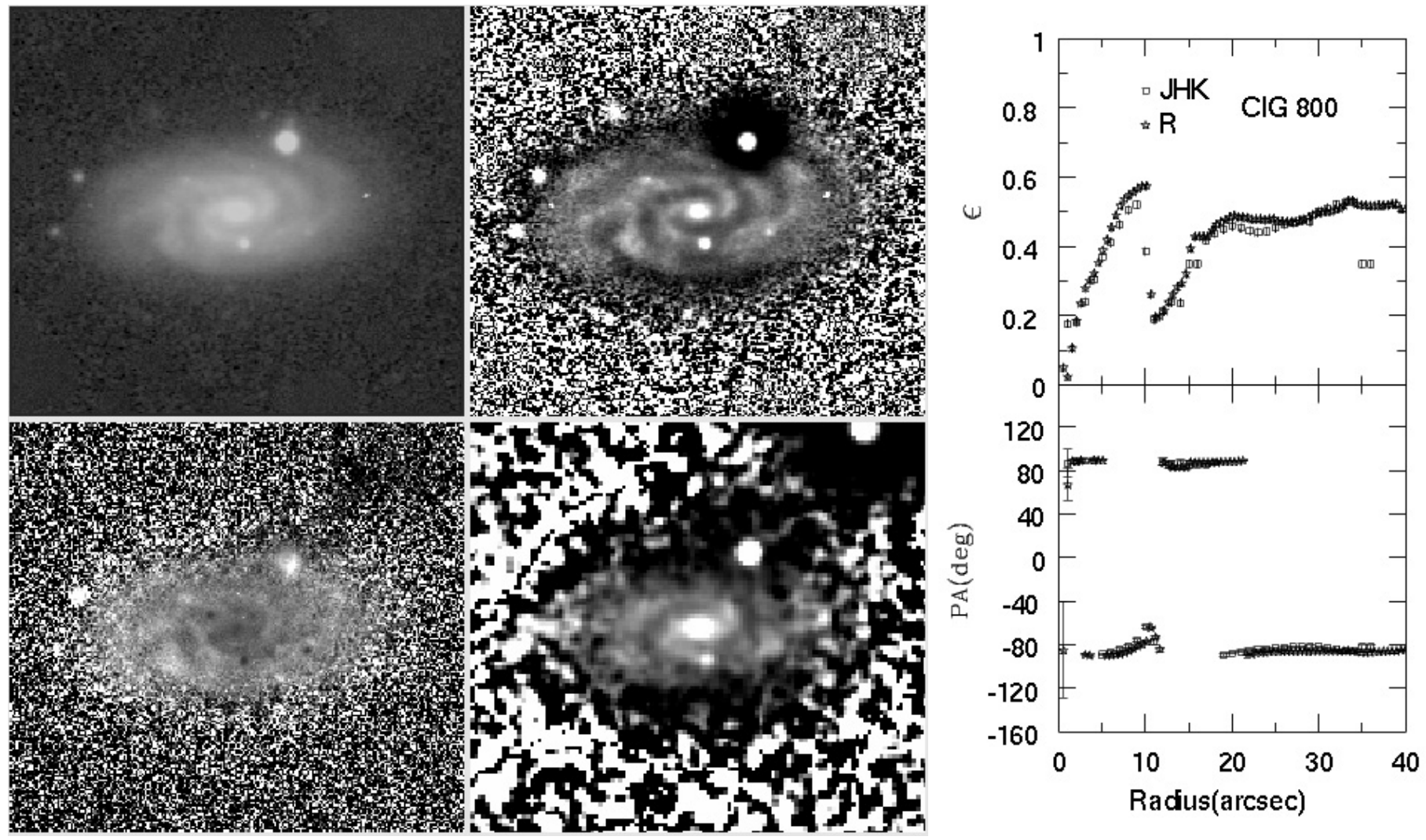

Fig. B.31. CIG 800 Mosaic. Same as Fig. 3. The major diameter of the galaxy in the optical images is 1.2 arcmin. 
H. M. Hernández-Toledo and S. Ortega-Esbrí: Broad-band BVRI photometry of isolated spiral galaxies, Online Material p 31
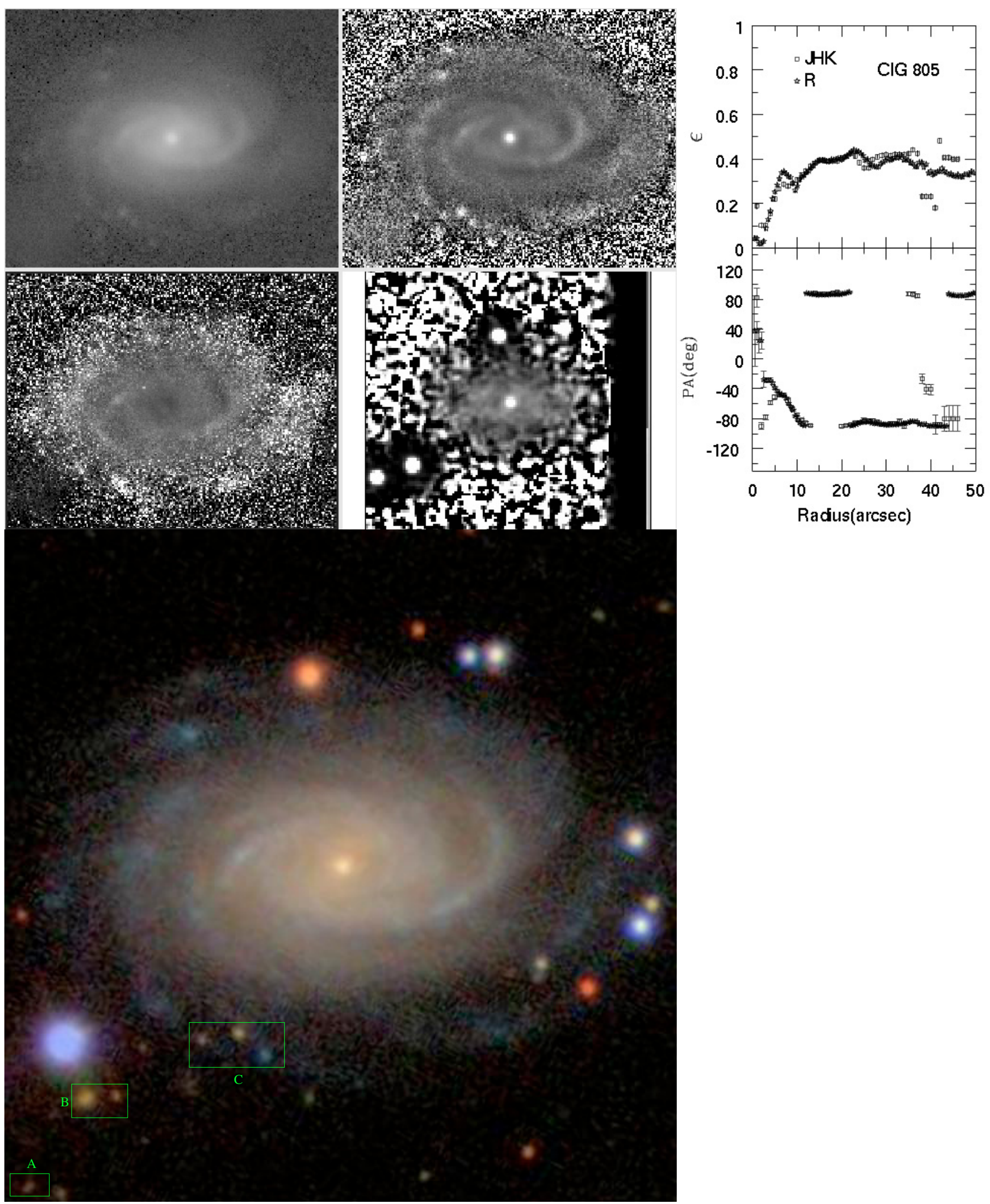

Fig. B.32. CIG 805 Mosaic. Same as Fig. 3. The major diameter of the galaxy in the optical images is 1.6 arcmin. 
H. M. Hernández-Toledo and S. Ortega-Esbrí: Broad-band BVRI photometry of isolated spiral galaxies, Online Material p 32
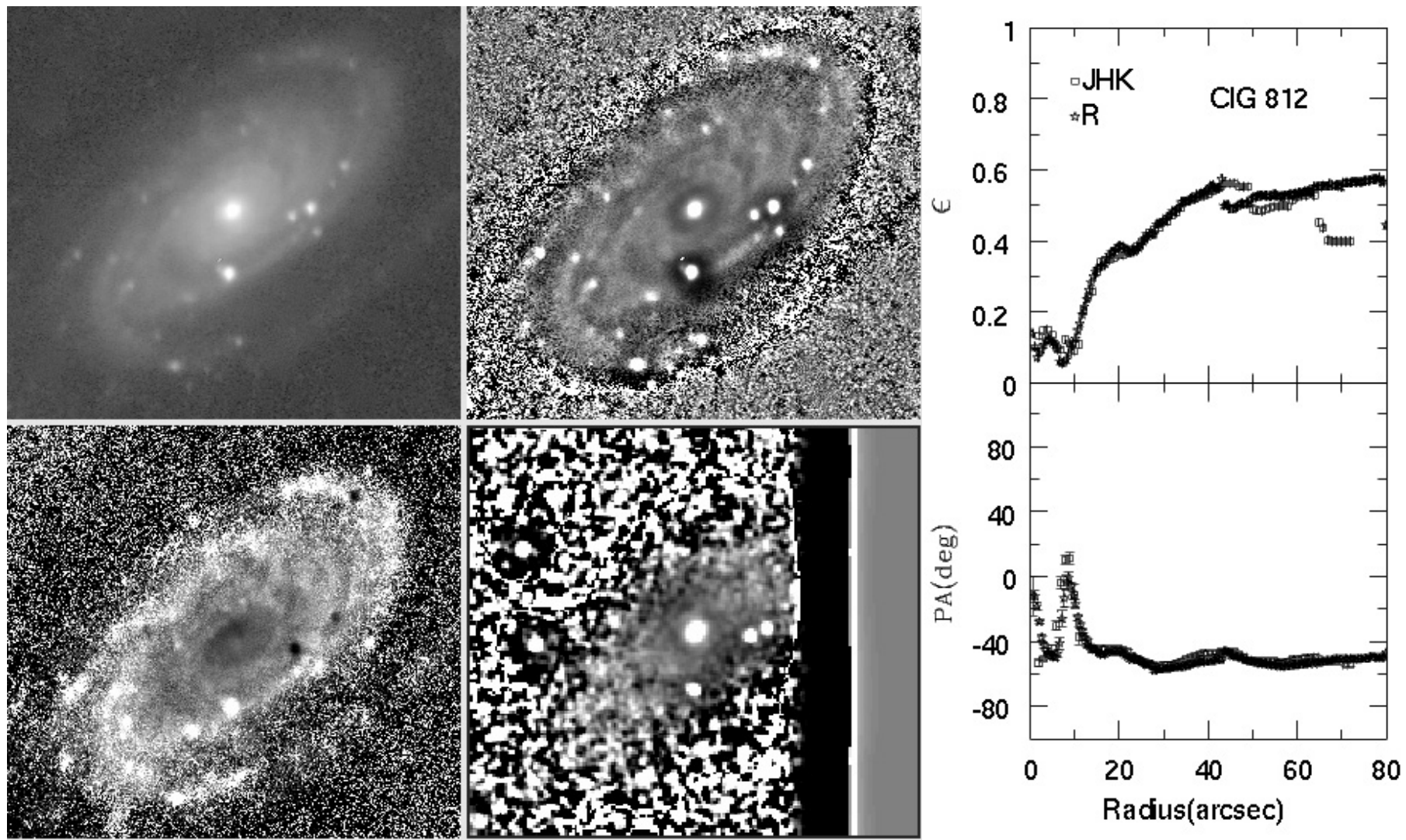

Fig. B.33. CIG 812 Mosaic. Same as Fig. 3. The major diameter of the galaxy in the optical images is 2.8 arcmin.
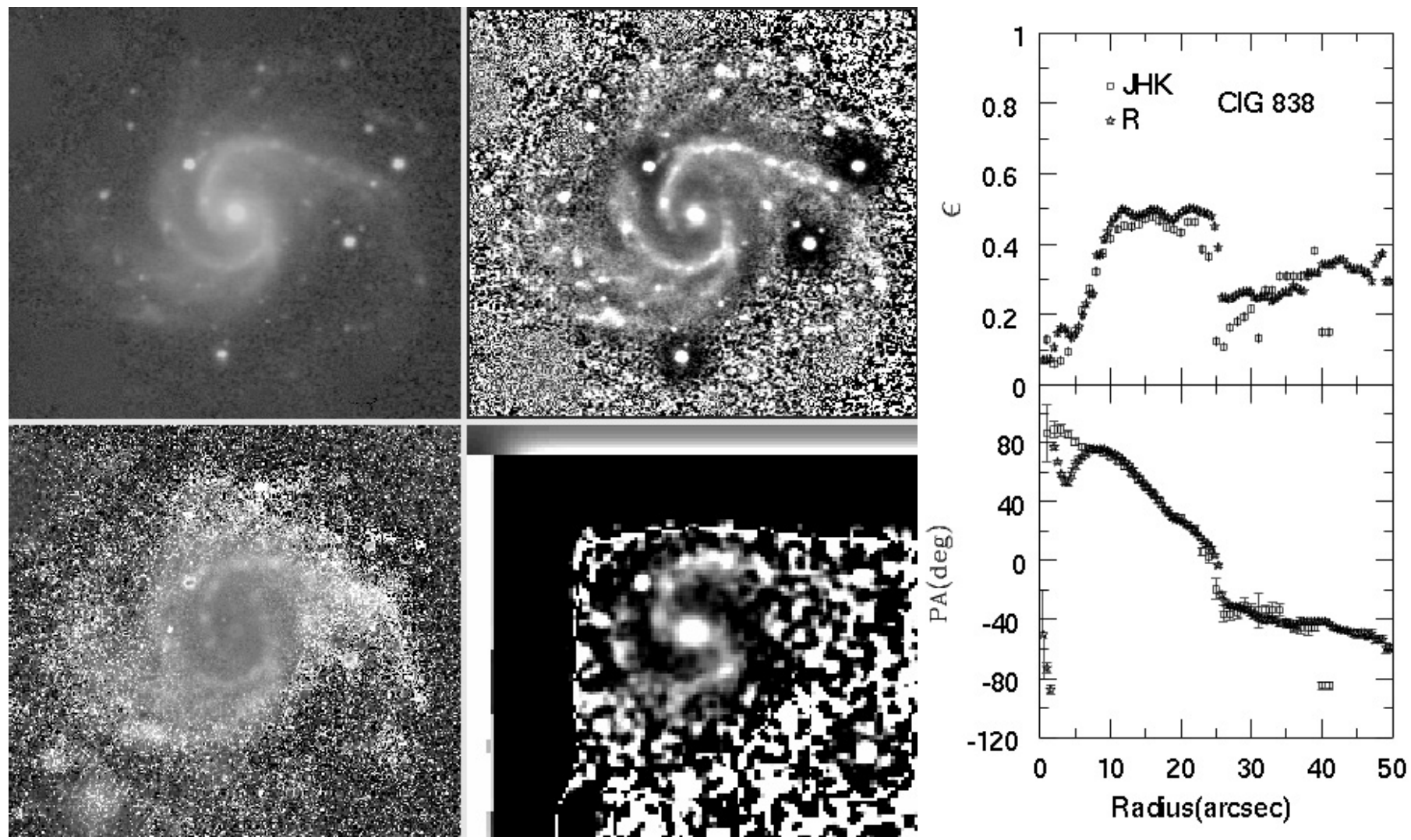

Fig. B.34. CIG 838 Mosaic. Same as Fig. 3. The major diameter of the galaxy in the optical images is 1.7 arcmin. 
H. M. Hernández-Toledo and S. Ortega-Esbrí: Broad-band BVRI photometry of isolated spiral galaxies, Online Material p 33
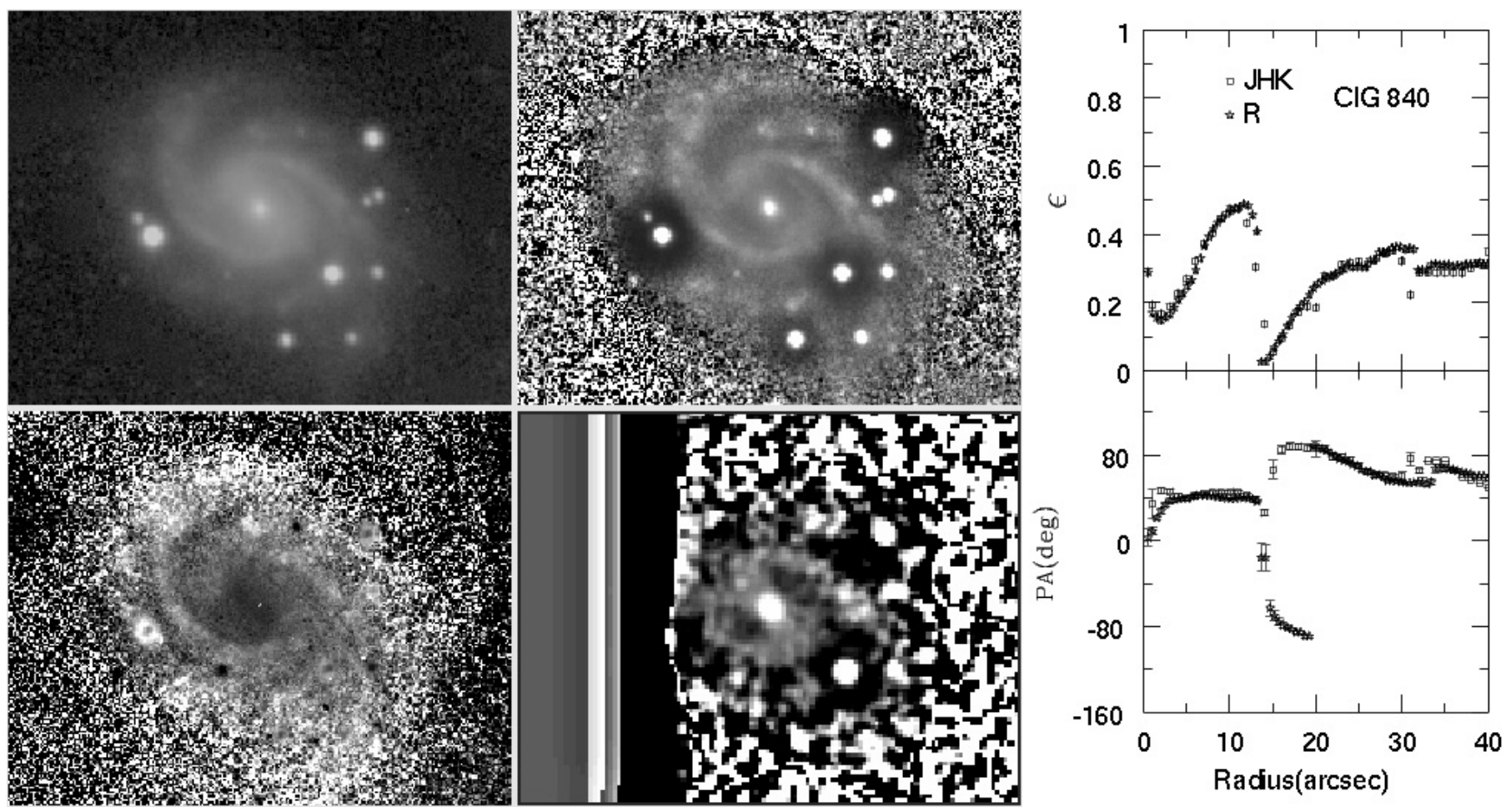

Fig. B.35. CIG 840 Mosaic. Same as Fig. 3. The major diameter of the galaxy in the optical images is 1.5 arcmin.
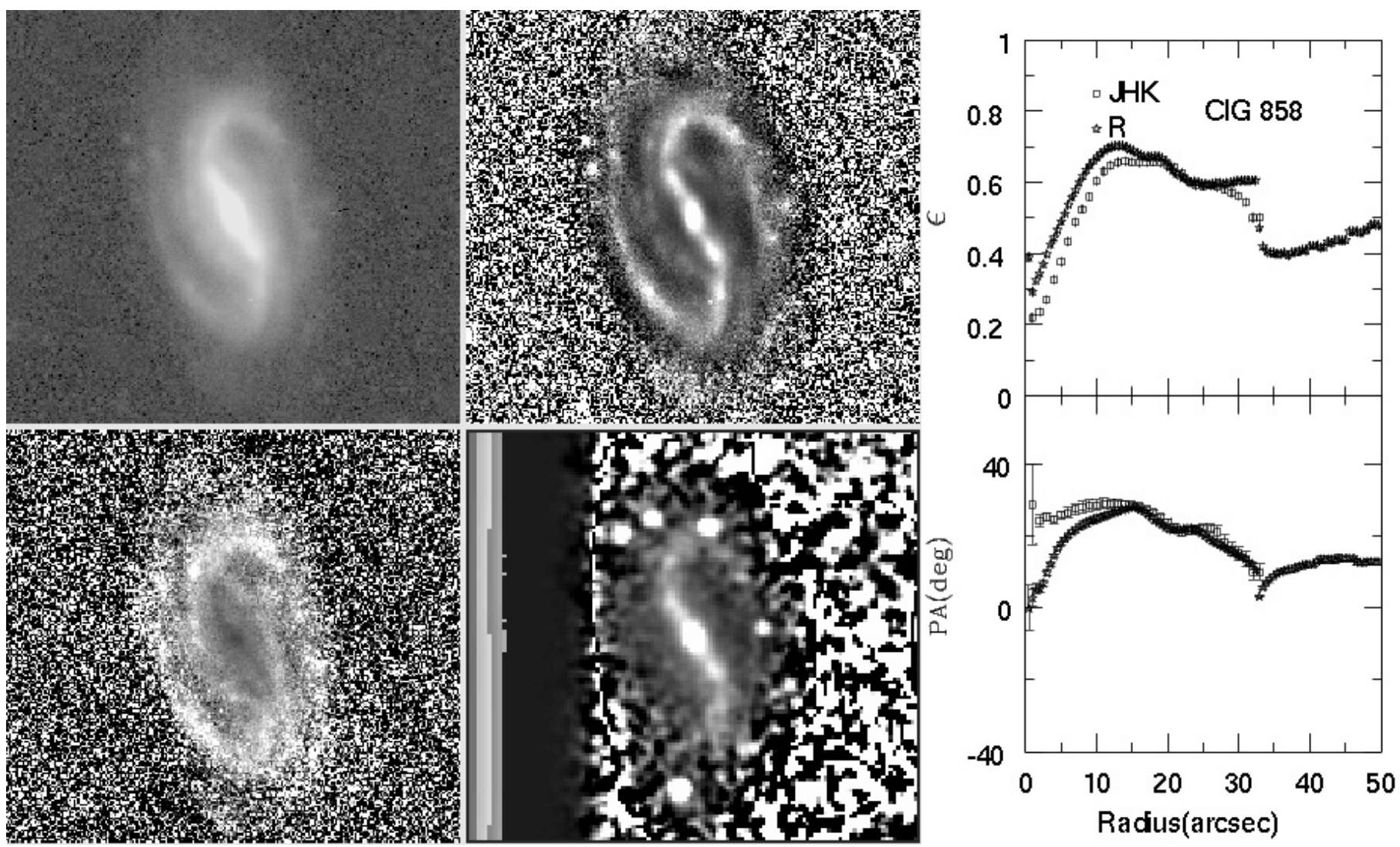

Fig. B.36. CIG 858 Mosaic. Same as Fig. 3. The major diameter of the galaxy in the optical images is 1.1 arcmin. 
H. M. Hernández-Toledo and S. Ortega-Esbrí: Broad-band BVRI photometry of isolated spiral galaxies, Online Material p 34
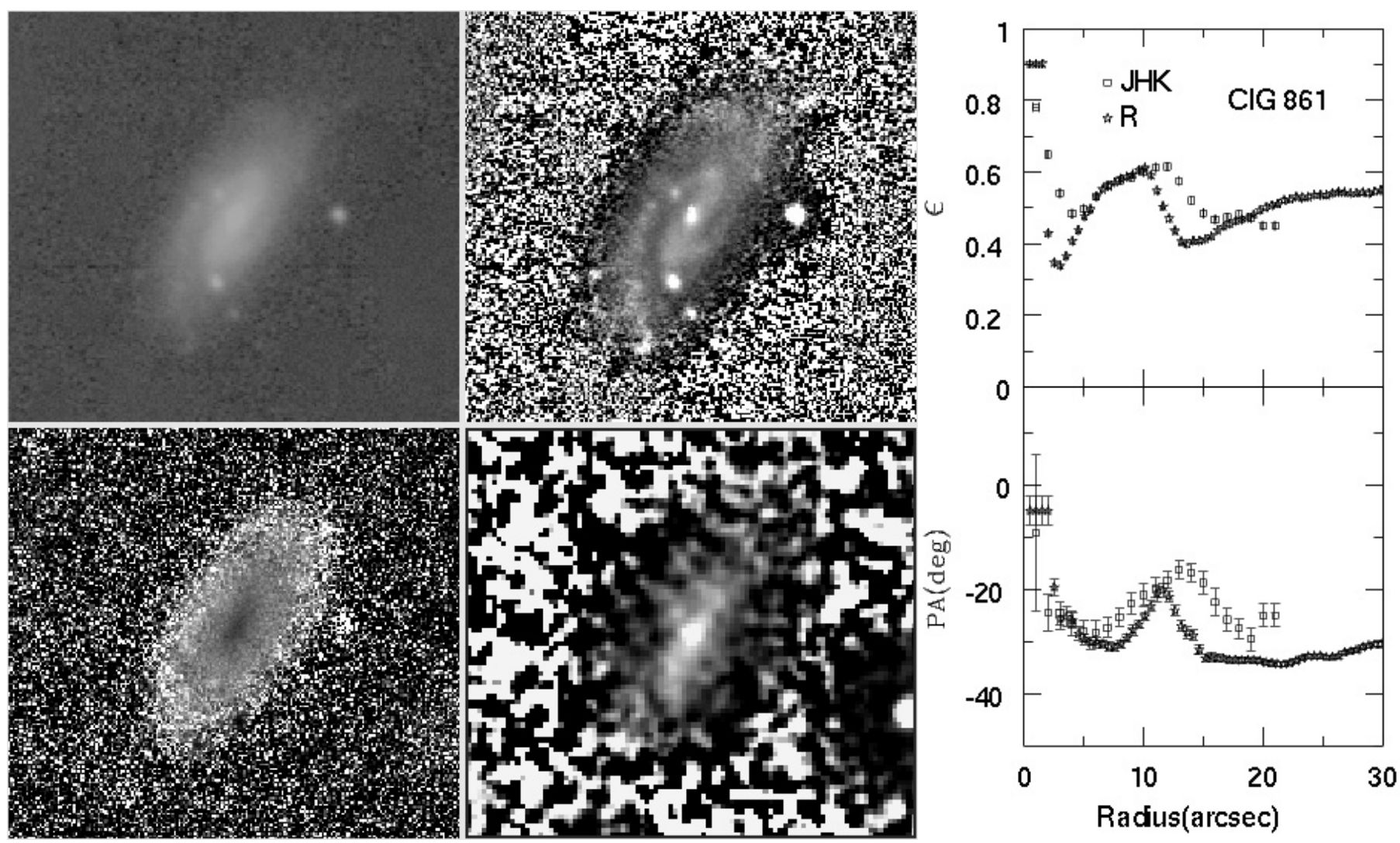

Fig. B.37. CIG 861 Mosaic. Same as Fig. 3. The major diameter of the galaxy in the optical images is 1.0 arcmin. 
H. M. Hernández-Toledo and S. Ortega-Esbrí: Broad-band BVRI photometry of isolated spiral galaxies, Online Material p 35
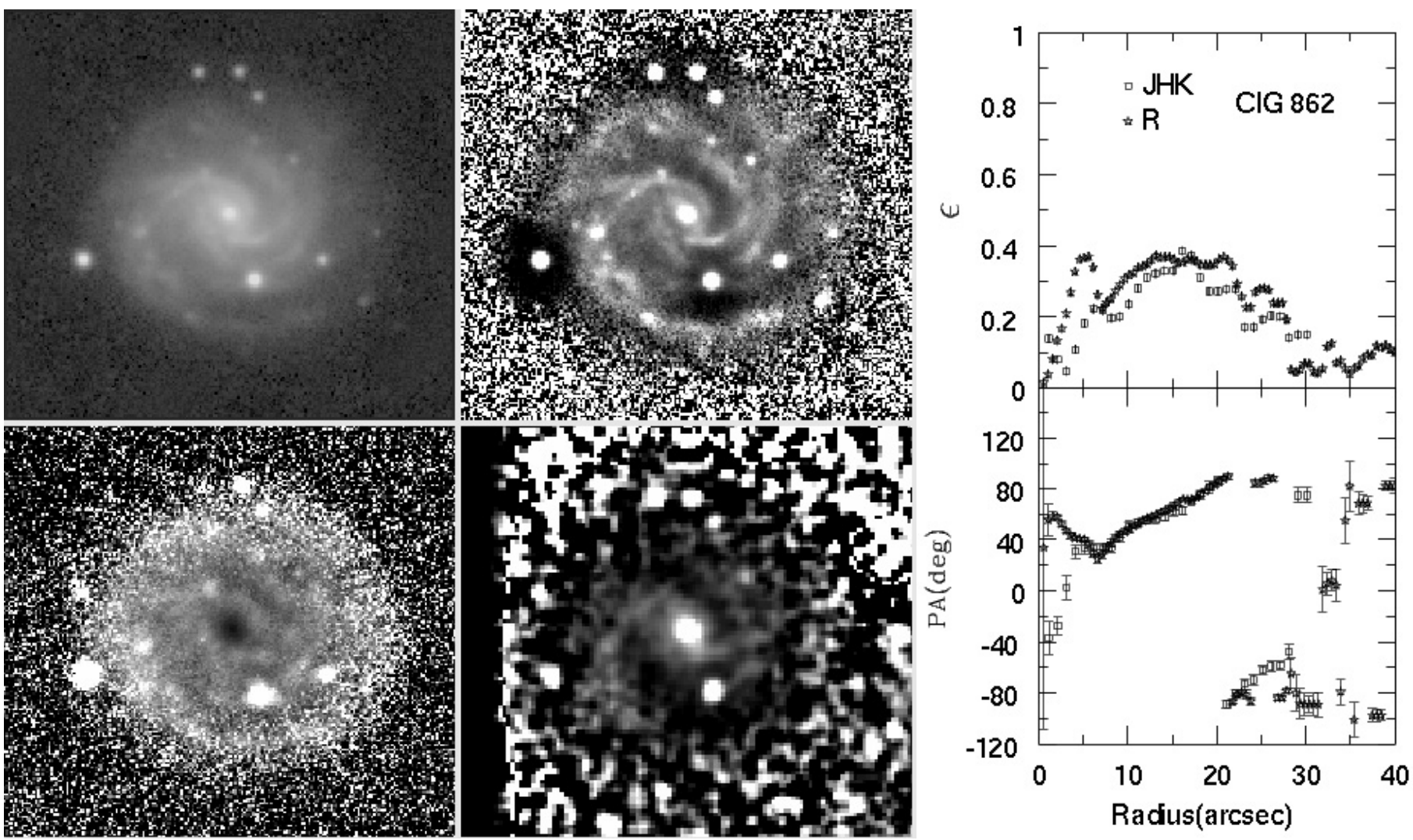

Fig. B.38. CIG 862 Mosaic. Same as Fig. 3. The major diameter of the galaxy in the optical images is 1.3 arcmin.
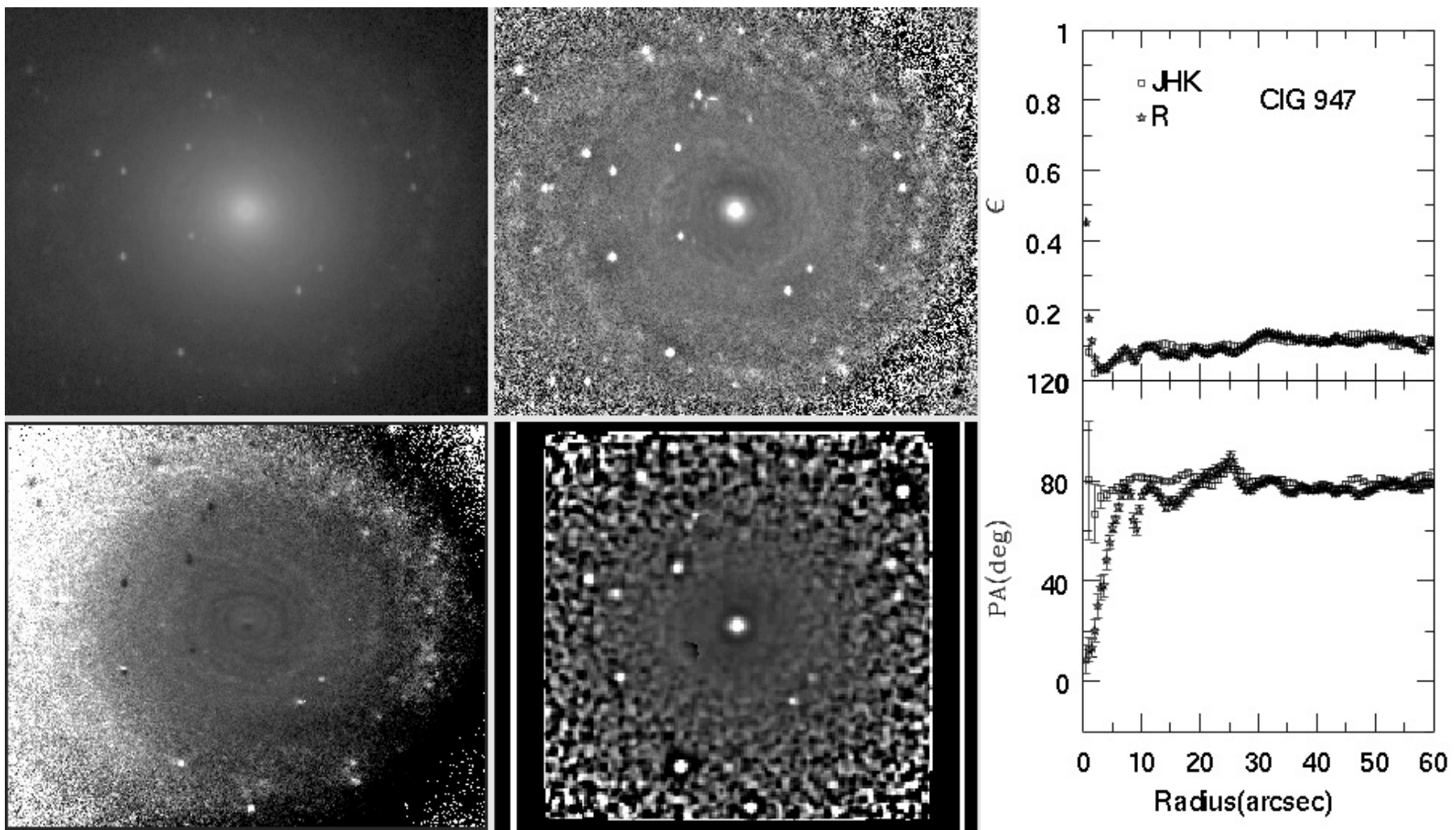

Fig. B.39. CIG 947 Mosaic. Same as Fig. 3. The major diameter of the galaxy in the optical images is 3.9 arcmin. 